University of Louisville

ThinkIR: The University of Louisville's Institutional Repository

Electronic Theses and Dissertations

$5-2020$

\title{
Microrobots for wafer scale microfactory: design fabrication integration and control.
}

Ruoshi Zhang

University of Louisville

Follow this and additional works at: https://ir.library.louisville.edu/etd

Part of the Electronic Devices and Semiconductor Manufacturing Commons, and the Nanotechnology Fabrication Commons

\section{Recommended Citation}

Zhang, Ruoshi, "Microrobots for wafer scale microfactory: design fabrication integration and control." (2020). Electronic Theses and Dissertations. Paper 3377.

https://doi.org/10.18297/etd/3377

This Doctoral Dissertation is brought to you for free and open access by ThinkIR: The University of Louisville's Institutional Repository. It has been accepted for inclusion in Electronic Theses and Dissertations by an authorized administrator of ThinkIR: The University of Louisville's Institutional Repository. This title appears here courtesy of the author, who has retained all other copyrights. For more information, please contact thinkir@louisville.edu. 


\title{
Microrobots For Wafer Scale Microfactory: Design, FAbrication, INTEGRATION AND CONTROL
}

\author{
By \\ Ruoshi Zhang \\ B.E., Tianjin University, 2012 \\ M.S., University of Texas at Arlington, 2015

\begin{abstract}
A Dissertation
Submitted to the Faculty of the

In Partial Fulfillment of the Requirements

for the Degree of

Doctor of Philosophy

in Electrical and Engineering

Department of Electrical and Computer Engineering

University of Louisville

Louisville, Kentucky
\end{abstract} \\ J.B. Speed School of Engineering of the University of Louisville
}

May 2020 
Copyright 2020 by Ruoshi Zhang

All Right Reserved 



\title{
Microrobots For Wafer Scale Microfactory: Design, FAbrication, INTEGRATION AND CONTROL
}

\author{
By \\ Ruoshi Zhang \\ B.E., Tianjin University, 2012 \\ M.S., University of Texas at Arlington, 2015
}

A Dissertation Approved on

April 17, 2020

by the following Dissertation Committee:

\begin{tabular}{c}
\hline Dan O. Popa \\
Shamus McNamara \\
\hline Shamus McNamara \\
Cindy K. Harnett \\
\hline Cindy K. Harnett \\
\hline John F. Naber \\
\hline
\end{tabular}

Keng H. Hsu 


\section{ACKNOWLEDGEMENTS}

I wish to express my deepest gratitude to my mentor Professor Dan Popa for his teaching during my entire PhD study, not only knowledge-wise, but also discovering my potentials, developing my professional skills, and shaping my mind. I would like to pay my special regards to Danming Wei and Brooke Hall, who have spent numerous hours of their time helping me assemble my microrobots. Without their efforts, I would not have what I have achieved today. I wish to show my gratitude to every cleanroom staff: Julia Aebersold, Evgeniya Moiseeva, Jasmin Beharic, Curt McKenna, Michael Martin, and Xiaojing Wang, for their help on my fabrication tasks. Without their dedicated work, I would not have a chance to finish my study. Speaking of making thing happen, I must mention the help I received from Douglas Jackson, who not only gave me practical suggestions, but also helped me with many hands-on tasks. I also wish to thank everybody who helped me with modeling, experiments, and writing: Professor Shamus McNamarra, Zhong Yang, Dr. Andriy Sherehiy, and Dr. M. Nasser Saadatzi.

Support and understanding from my family are the foundation of my achievement today.

I owe them a lot for the years of my absence, and I hope my tiny achievement would provide them a little comfort.

The dissertation and the final defense were finished during a very special time, since the corona virus outbreak grounded everybody at home. Here I must thank my dearest fiancé, Maoyin Zhang, who gave me numerous supports from home. 
Last but not least, I must thank the National Science Foundation, who provided funding for our research. Needless to say, without funding it is extremely difficult to conduct such research. The grand numbers are \#CMMI 1734383 and \#IIS 16331 


\begin{abstract}
Microrobots For WAFER SCAlE MicrofaCtory: DeSign, FABRiCATION, InTEGRATION AND CONTROL

Ruoshi Zhang
\end{abstract}

April 17, 2020

Future assembly technologies will involve higher automation levels, in order to satisfy increased micro scale or nano scale precision requirements. Traditionally, assembly using a top-down robotic approach has been well-studied and applied to micro-electronics and MEMS industries, but less so in nanotechnology. With the bloom of nanotechnology ever since the 1990s, newly designed products with new materials, coatings and nanoparticles are gradually entering everyone's life, while the industry has grown into a billion-dollar volume worldwide. Traditionally, nanotechnology products are assembled using bottomup methods, such as self-assembly, rather than with top-down robotic assembly. This is due to considerations of volume handling of large quantities of components, and the high cost associated to top-down manipulation with the required precision. However, the bottom-up manufacturing methods have certain limitations, such as components need to have pre-define shapes and surface coatings, and the number of assembly components is limited to very few. For example, in the case of self-assembly of nano-cubes with origami design, post-assembly manipulation of cubes in large quantities and cost-efficiency is still challenging. 
In this thesis, we envision a new paradigm for nano scale assembly, realized with the help of a wafer-scale microfactory containing large numbers of MEMS microrobots. These robots will work together to enhance the throughput of the factory, while their cost will be reduced when compared to conventional nano positioners. To fulfill the microfactory vision, numerous challenges related to design, power, control and nanoscale task completion by these microrobots must be overcome. In this work, we study three types of microrobots for the microfactory: a world's first laser-driven micrometer-size locomotor called ChevBot, a stationary millimeter-size robotic arm, called Solid Articulated Four Axes Microrobot (sAFAM), and a light-powered centimeter-size crawler microrobot called SolarPede. The ChevBot can perform autonomous navigation and positioning on a dry surface with the guidance of a laser beam. The sAFAM has been designed to perform nano positioning in four degrees of freedom, and nanoscale tasks such as indentation, and manipulation. And the SolarPede serves as a mobile workspace or transporter in the microfactory environment. 


\section{TABLE OF CONTENTS}

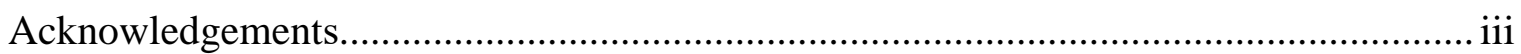

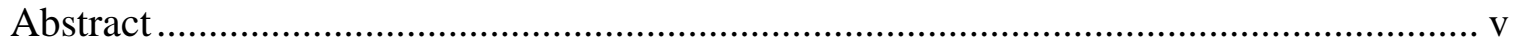

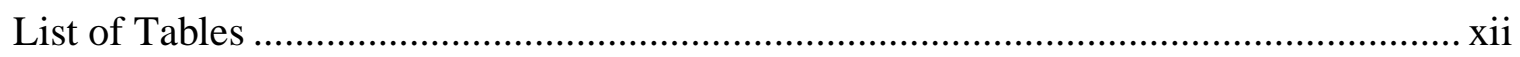

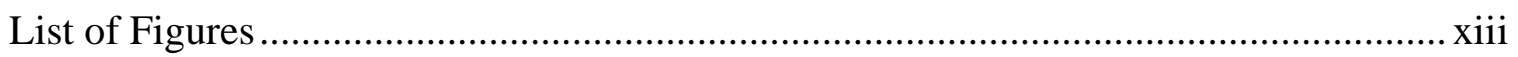

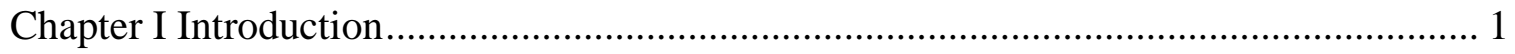

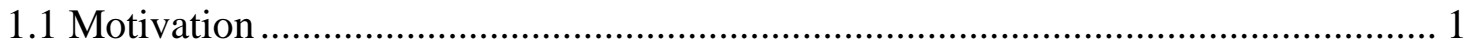

1.1.1 Robotic Applications to Micro and Nano-Scale Manipulation ........................ 1

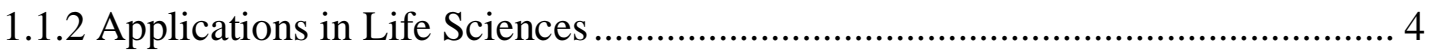

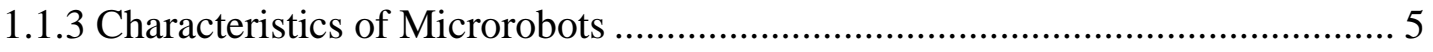

1.1.4 Silicon MEMS-based microrobots ........................................................... 7

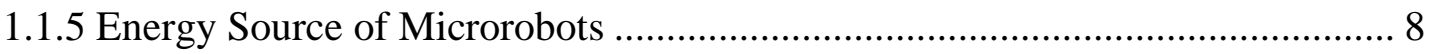

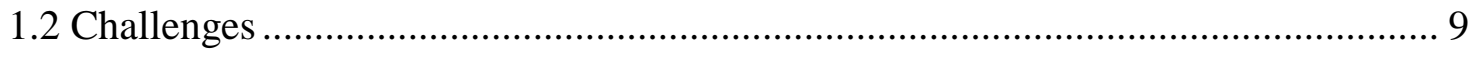

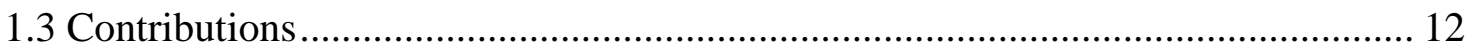

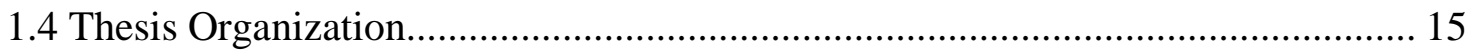

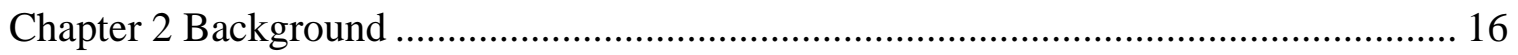

2.1 Microrobots ............................................................................................. 16

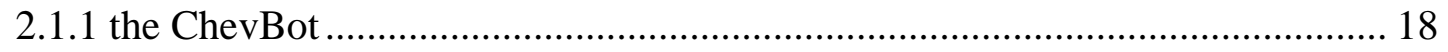


Chapter 3 The Laser Driven Microrobot - ChevBot.................................................. 26

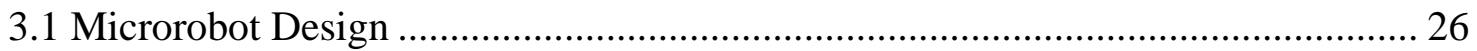

3.1.1 Principle of Operation .......................................................................... 27

3.1.2 Design Efforts Toward Straight Trajectory Locomotion ............................. 28

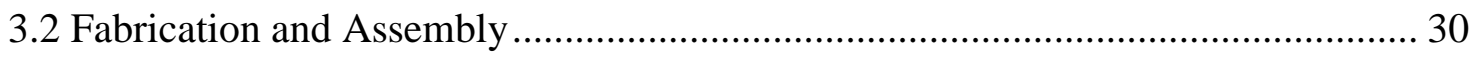

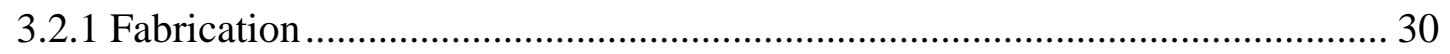

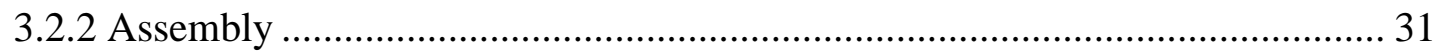

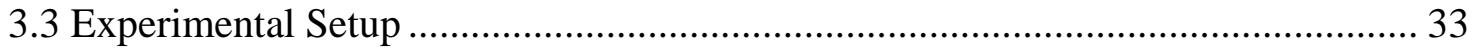

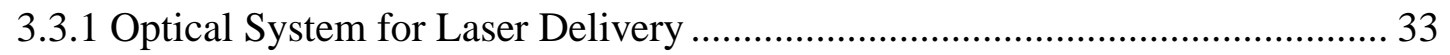

3.3.2 Vision Acquisition and Automated Motion Tracking ................................. 35

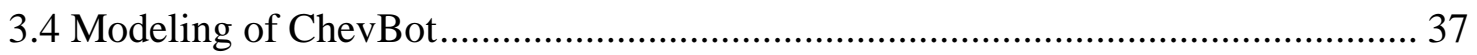

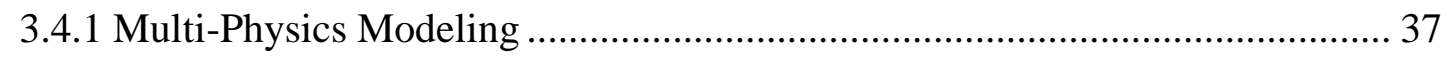

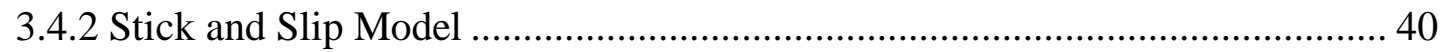

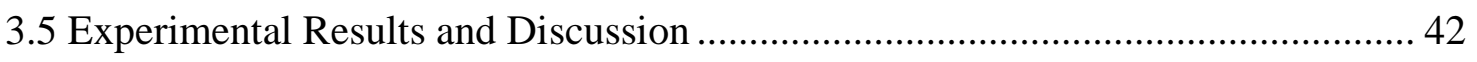

3.5.1 Experiments with tethered robots for model identification ........................... 43

3.5.2 Experiments with mobile ChevBots ...................................................... 45

Chapter 4 The Solid Articulated Four Axes Microrobot.............................................. 48 
4.1 Design of sAFAM Microrobot 48

4.1.1 Microrobot Components 48

4.1.2 Assembly Methodology 51

4.2 Fabrication and Assembly 52

4.2.1 Fabrication 52

4.2.2 Assembly 54

4.3 Finite Element Analysis of Microrobot. 56

4.3.1 The Experiment Assisted Simulation Method .............................................. 56

4.3.2 Stiffness Analysis of the Thin Beam Spring and the Serpentine Spring .......... 57

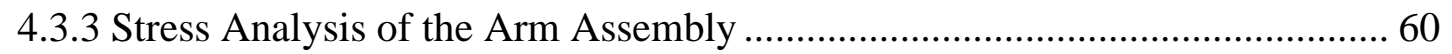

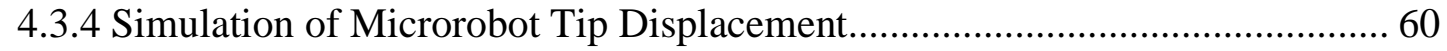

4.4 Experimental Results: Microrobot Workspace .............................................. 62

4.4.1 FEA Model Validation using Optical Microscopy ...................................... 62

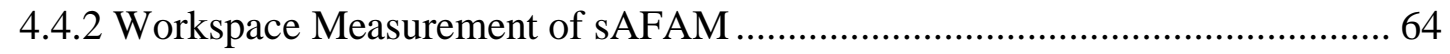

4.5 Experimental Results: Microrobot Resolution and Repeatability ....................... 65

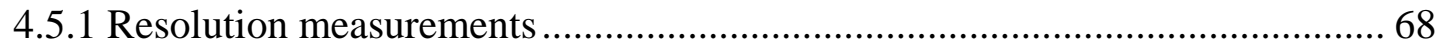

4.5.2 Repeatability Measurement Experiment ............................................... 70

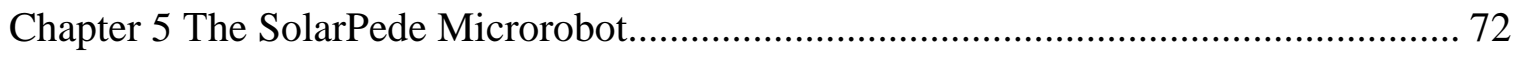

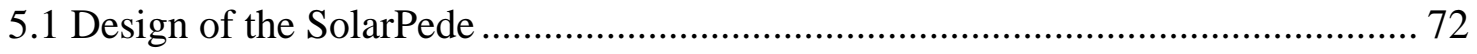

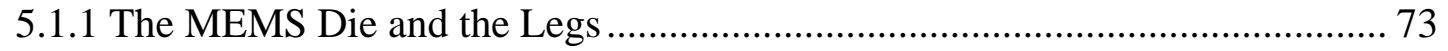


5.1.2 Principle of Operation

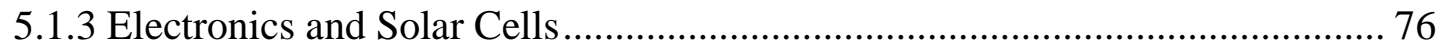

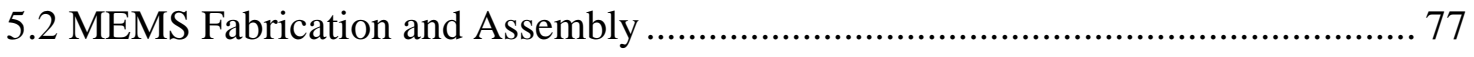

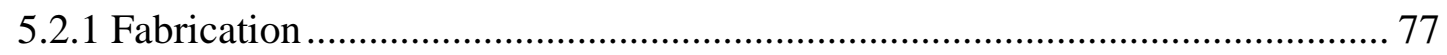

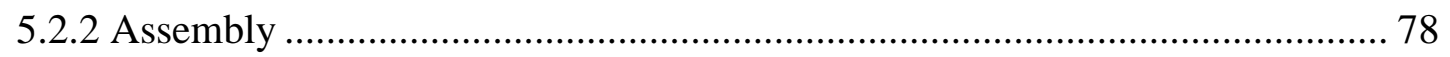

5.3 Modeling and Analysis of the Microrobot ....................................................... 79

5.3.1 Stick and Slip Model and Legs Displacement.......................................... 79

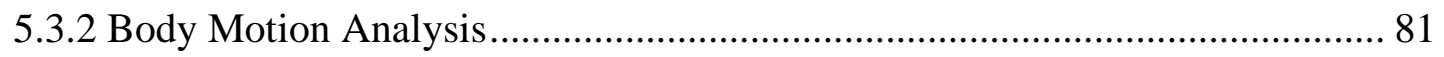

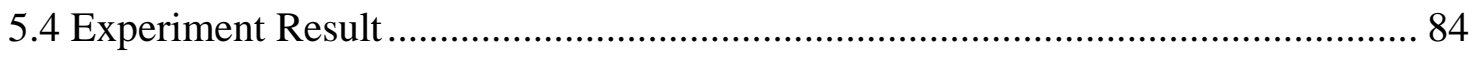

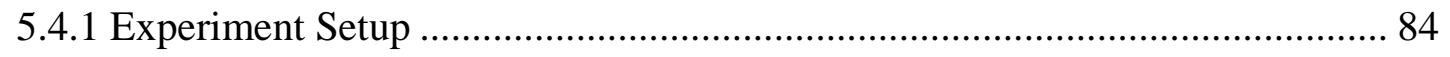

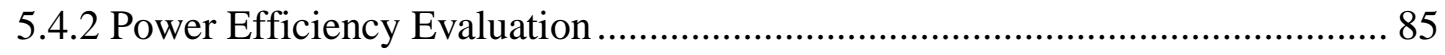

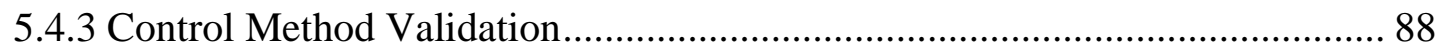

5.4.4 “Belly-up” Payload Motion Testing Powered by Solar Simulator................... 89

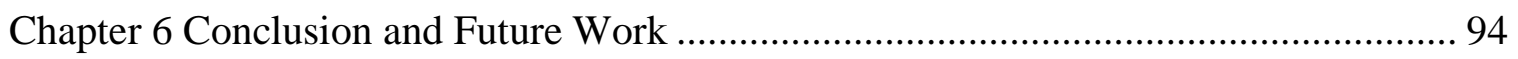

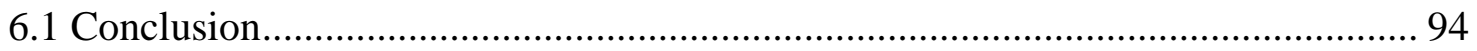

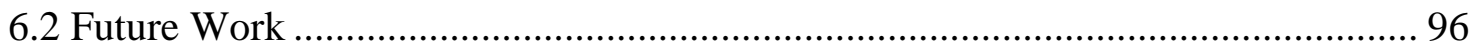

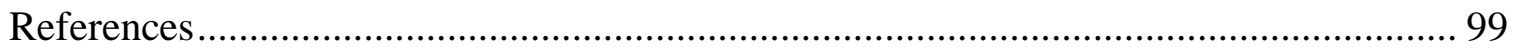

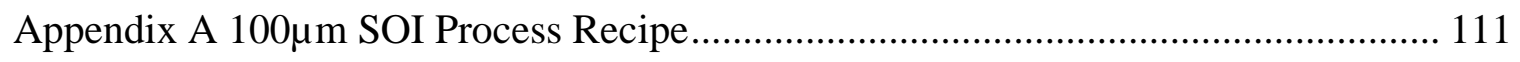

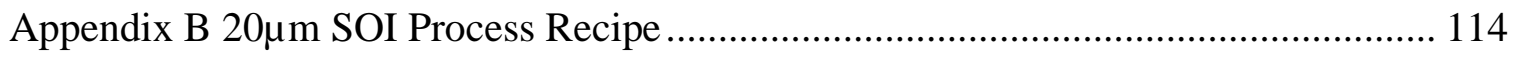

Appendix C ChevBot's Tracking Experiment Design............................................. 116 
C.1 Fix the Stage, or the Laser?

C.2 Experiment Hardware Configuration .............................................................. 119

C.3 Controller Program Design......................................................................... 120

C.3.1 Inverse Image Jacobian Calculation ………………................................ 120

C.3.2 ChevBot's Tracking Program .................................................................. 122

Appendix D SolarPede's Hardware Design................................................................ 123

D.1 Power Board Design and Construction ............................................................ 123

D.2 Bluetooth MCU Selection and Configuration ................................................... 126

D.3 Control Board Design and Construction ........................................................... 129

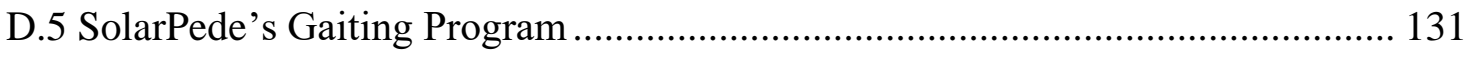

D.6 List of Major Electrical Components Used in SolarPede Prototype .................... 135

Appendix E List of Publications ............................................................................. 136

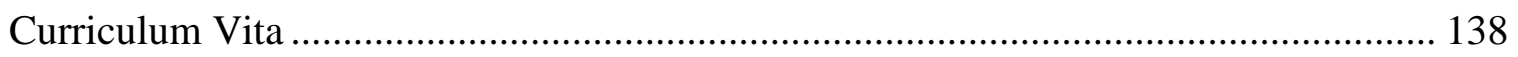




\section{LIST OF TABLES}

Table I Values of the Constant Used in the Simulation.............................................. 38

Table II Untethered Locomotion Measurement Results ........................................... 47

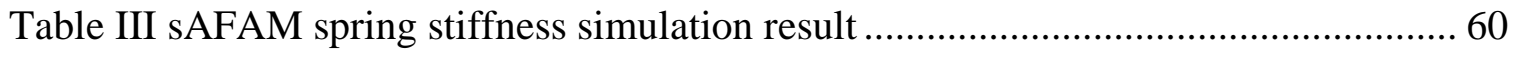

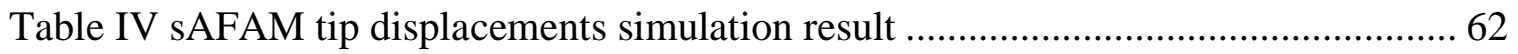

Table V Power consumption of a single thermal actuator from 5 to $20 \mathrm{~V}$....................... 86

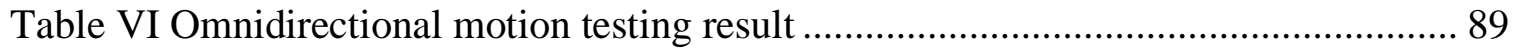

Table VII Recorded payload displacement within 30s ........................................... 92 


\section{LIST OF FIGURES}

Figure 1-1 Two examples of in situ SEM manipulation products .................................. 3

Figure 1-2 Various types of "regular-sized" robots ................................................... 6

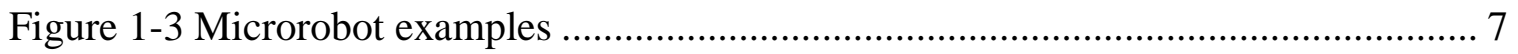

Figure 1-4 Examples of the electromagnetic coils used in controlling of the magnetic-

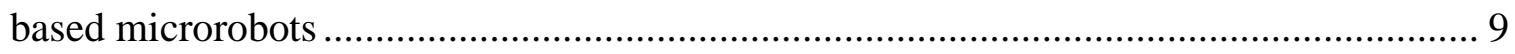

Figure 1-5 3D rendering of proposed microfactory .............................................. 14

Figure 3-13D drawing of ChevBot with critical dimensions ................................... 27

Figure 3-2 Illustration of ChevBot's stick-and-slip locomotion ................................ 28

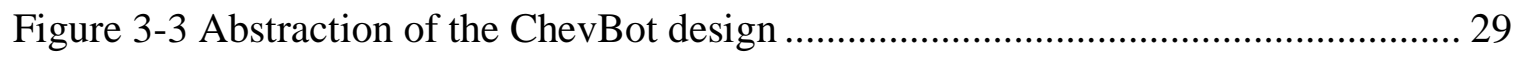

Figure 3-4 Mask layout of ChevBot body and its dimple assembly ............................. 30

Figure 3-5 The NeXus microassembly station used to assemble ChevBots................... 32

Figure 3-6 Schematic of laser delivery and vision acquisition system ......................... 35

Figure 3-7 Control scheme of the combined laser-beam robot motion ......................... 35

Figure 3-8 Block diagram of simulation model .................................................. 38 
Figure 3-9 Free body diagram of ChevBot - sideview

Figure 3-10 Changes of the position of center of mass (x) as a function of time for different values of repetition frequency and irradiance

Figure 3-11 Laser power vs. thermal actuator displacement 44

Figure 3-12 ChevBot's trajectory from 5 different experiments 46

Figure 4-1 Design illustration of sAFAM

Figure 4-2 NEXUS microassembly station. 55

Figure 4-3 Side view of the assembled sAFAM arm 56

Figure 4-4 Original position vs. actuated position 58

Figure 4-5 Spring stiffness simulation setup 59

Figure 4-6 End-effector displacement experiment result 63

Figure 4-7 Measured sAFAM workspace. 65

Figure 4-8 Images of two different experimental set up, and schematics of 4 different setup for the measurements 66

Figure 4-9 Displacement of the sAFAM arm in the $\mathrm{X}$ direction (translation) as a function of time and actuation voltage 68

Figure 4-10 Resolution variation along four DOFs 69 
Figure 4-11 Repeatability variation along four DOFs

Figure 5-1 Side-view illustration of SolarPede's construction ..................................... 72

Figure 5-2 System level functionality abstraction of SolarPede.................................. 73

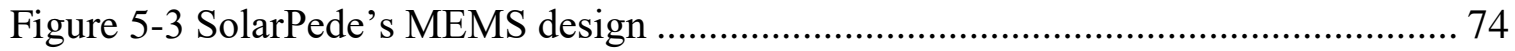

Figure 5-4 Theoretical vectors of holonomic motion methodology for SolarPede

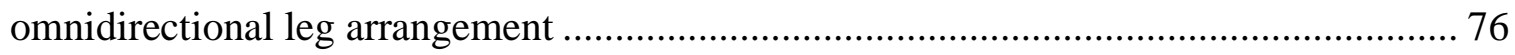

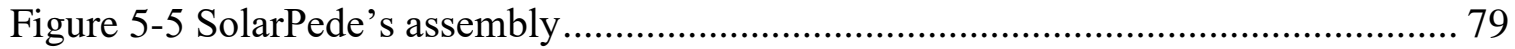

Figure 5-6 Mass-spring-damper model of the SolarPede's leg and body........................ 80

Figure 5-7 Microrobot body velocity vs. leg actuation frequency, and voltage .............. 81

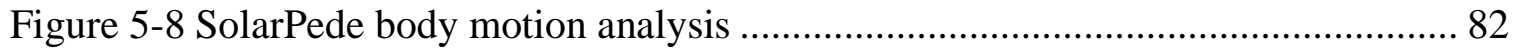

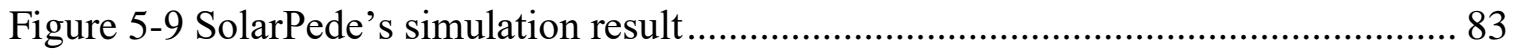

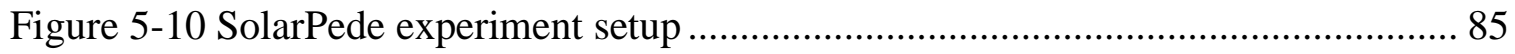

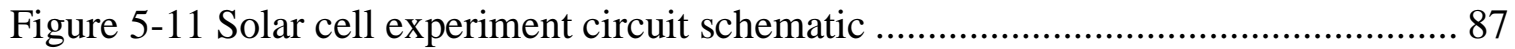

Figure 5-12 Assembled and packaged SolarPede die carrying a payload ...................... 89

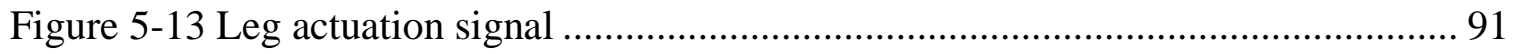

Figure 5-14 Trajectory of the payload displacement .............................................. 93

Figure 6-1 sAFAM with support in SEM chamber .............................................. 98 
Figure C-1 Schematic of two approaches 116

Figure C-2 Gaussian beam illustration 117

Figure C-3 Illustration of an ideal Gaussian beam on ChevBot's thermal actuators ...... 118

Figure C-4 Stage and smart camera initialization

Figure C-5 Smart camera pixel position acquisition 121

Figure C-6 Stage random step generation

Figure D-1 Circuit schematic of the top board 124

Figure D-2 Front side of the top PCB 124

Figure D-3 Bottom side of the top PCB. 125

Figure D-4 Completed power board 126

Figure D-5 BL652 Bluetooth MCU module 126

Figure D-6 Schematic of the control board. 128

Figure D-7 Front layer of the control board. 129

Figure D-8 Bottom side of the control board 130

Figure D-9 Aluminum fixture of the control board to help wire-bonding. 130

Figure D-10 Fully assembled control board, with MEMS die wire bonded. 131 


\section{CHAPTER I INTRODUCTION}

\subsection{Motivation}

The word robot originates from the Czech playwriter Karel Capek’s science-fiction play “Rossum's Universal Robots”, meaning “forced labor" [1]. This idea of a robot represents people's wish to have man-made agents that are capable of conducting numerous works on behave of human beings, such as tasks with repetitive nature, tasks involving dangerous situations, or interacting with humans. With the rapid development of science and technology, many of the above goals can be fulfilled by different types of robots, and not just humanoid robots. A more commonplace example is that human workers assisted by industrial robots in car manufacturing facilities to improve efficiency and accuracy, while reduce work injuries. At science fiction fairs, guests are welcomed by social reception robots and receive answers from them. And, at the scene of a disaster, mobile rescue robots traverse dangerous environments to look for survivors. Besides above applications, where robots serve as replacements and enhancements for humans, exploring micro to nano meter scale world is one where robots are necessary since humans have capabilities limitations due to size.

\subsubsection{Robotic Applications to Micro and Nano-Scale Manipulation}

Researchers have been working on manipulating micro and nano scale objects for different bio and nano applications for the last four decades. One of the most important applications of such multi-scale manipulation is to assemble micro and nano structures that 
are too difficult or impossible for human hands to achieve. In this context, the bottom-up manufacturing method represented by self-assembly technology was widely studied [2] [3] [4] [5] [6] [7], and shown capable to provide hundreds and thousands of preprocessed building blocks, while also limited in its abilities to further manipulate those building blocks to arrange them into a final product. To cover this gap, researchers have pursued traditional approaches, such as motorized stages, robotic arms, or atomic force microscopes (AFM), however they all suffer from severe disadvantages and limitations. Given the micro-scale product they manipulate, the above machines need to be made with supreme accuracy, which leads to prohibitively high costs. Also, they are less space-efficient since the size of such equipment are bulky comparing with the part they process. As a result, scaling the microfactory to realize parallel processing of nanoscale component is not feasible. Environmental control also poses challenges to this solution, since most of the micro and nano scale manipulation requires a clean room environment so that they are free of dust particle influence and provide high yield. Indeed, even the bottom-up method of self-assembly relies on carefully designed mechanics or conditions (magnetic, electrical, etc.) and specific environments (wet, chemical, etc.) [8].

The manufacturing community has been actively looking for solutions of future micro and nano manufacturing that are scalable in sizes and cost. According to Qin [9], micromanufacturing can be categorized into two major types: MEMS-based, and nonMEMSbased. Micro Electromechanical Systems (MEMS) - based technologies utilize the conventional silicon-based fabrication process, with many years of development, already commercially successful and widely available. Silicon MEMS fabrication includes standard processes such as deposition, photolithography, and chemical etching. Due to its 
process limitations, it lacks the capability of achieving sophisticated 3D geometries, it is difficult to down-size the tools, and resource consumption and manufacturing environment requirement is high. On the other hand, nonMEMS-based manufacturing processes include many novel approaches, such as laser cutting, additive manufacturing, electrical discharge machining, and others. While these approaches can incorporate a variety of 3D geometries and materials into manufactured components, they too have limitations of volume due to their serial nature.

In situ electron microscopy (SEM) has a wide range of applications. For example, in biology, samples may need to be interacted with, operations such as pushing, peeling, or moving are desired. In semiconductor research, circuit samples may need to be probed under SEM to test the performance. Most of current manipulators used in nano and micro manipulation are based on step motors and piezo actuators, with a conventional mechanical housing and probe [10]. Very often the vacuum chamber of a SEM is confined, while those manipulators are centimeter-sized thus only limited amount of them can fit into the chamber, as shown in Figure 1-1.

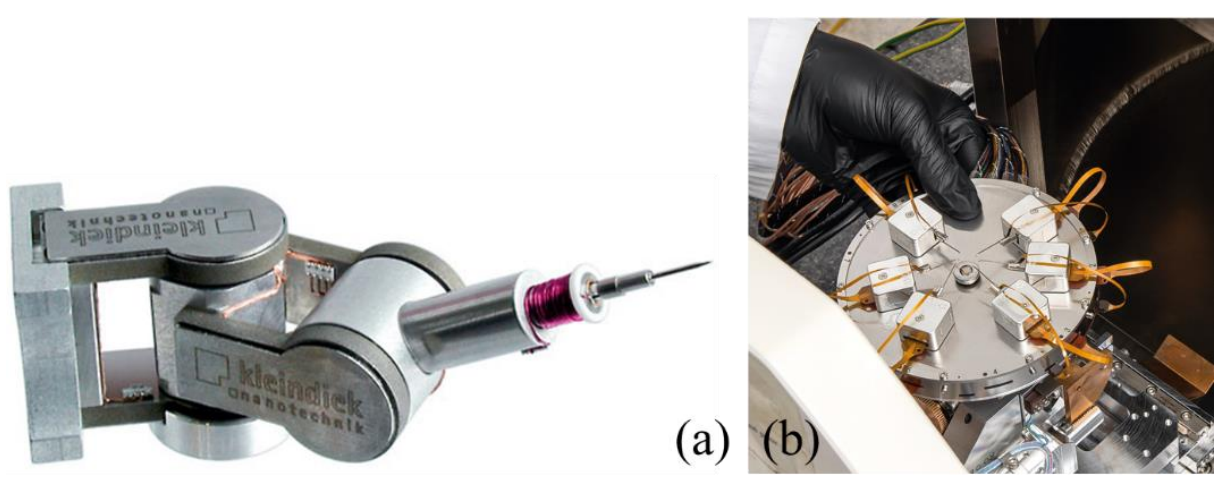

Figure 1-1 Two examples of commercially available in-situ SEM manipulation products. (a) MM3A-EM manipulator from Kleindiek® [11]. (b) miBot from IMINA® [12].

One elegant solution to bridge the gap between the macro and nano worlds is to create micro-scale robots to conduct micro and nano scale manufacturing and assembly tasks in 
a microfactory. Similar to the real-world application of robots building cars in a factory, many researchers are interested in applying such ideas into micro and nano scale manipulation [13] [14] [15]. A microfactory is one environment in which micro or nanoscale structures should be made by micro-scale robots having comparable sizes to the structure itself. Within such a factory, fundamental building blocks can be manipulated, processed and assembled by microrobot in a similar way to an assembly line in a conventional factory. Under such arrangement, the miniaturized assembly line improves efficiency in resource, space, and cost.

\subsubsection{Applications in Life Sciences}

Microrobot's nature of small size makes it advantageous for use in life sciences and medicine applications. Precision in-body drug delivery is one of such on-going research

directions. A free moving agent navigating within human blood vessels can release a precise dosage of drugs, with many potential advantages over traditional oral administration, such as avoiding side-effects by supplying medication to a desired target site. Furthermore, the dosage concentration can be optimized be controlling the release time and conditions [16]. If equipped with actuation mechanisms, microrobots can also be used to perform microsurgery within human body, which effectively reduces invasiveness thus reduce pain. For example, microrobots can be used to clean clots attached on blood vessels [17].

Besides drug delivery, and in-vivo surgery, microrobots can be used in-vitro to assist with manipulation tasks of biological materials. Cell manipulation includes precisely control the location of individual cells, probing, and stimulation [18]. Cell manipulation use to achieved with pipettes; with evolving technology, new means were developed such 
as optical trapping, micro-fluidics conjunction with MEMS devices, and microrobotics. Among other types, the magnetic field driven microrobots gain its popularity due to a couple of factors. The power and control can achieve full wireless, the magnetic field does not affect cells biology activities, and the operation does not introduce physical change into the biology environment [19] [20].

\subsubsection{Characteristics of Microrobots}

According to another common definition, a robot is a mechanism that is capable of sensing, reasoning, and actuating. A typical robot, such as ATLAS [21] shown in Figure 1-2 (a), fits people's general impression of a robot: it looks like a human and also try to behave like a human. From scientific perspective, it integrates all three elements together in its body. For instance, multiple sensors such as a Light Detection and Ranging sensor (LIDAR) are equipped in its head to identify obstacles along the path, and hydraulic actuators drive the legs to walk.

The rest of the pictures in Figure 1-2 depict other types of robots. In (b), Kuka robots are used to assemble cars. In (c), Humonid robot PKD can speak to people with humanlike face expressions. Although the Wall-E robot is fictional, it also contains the three main features of a robot. 


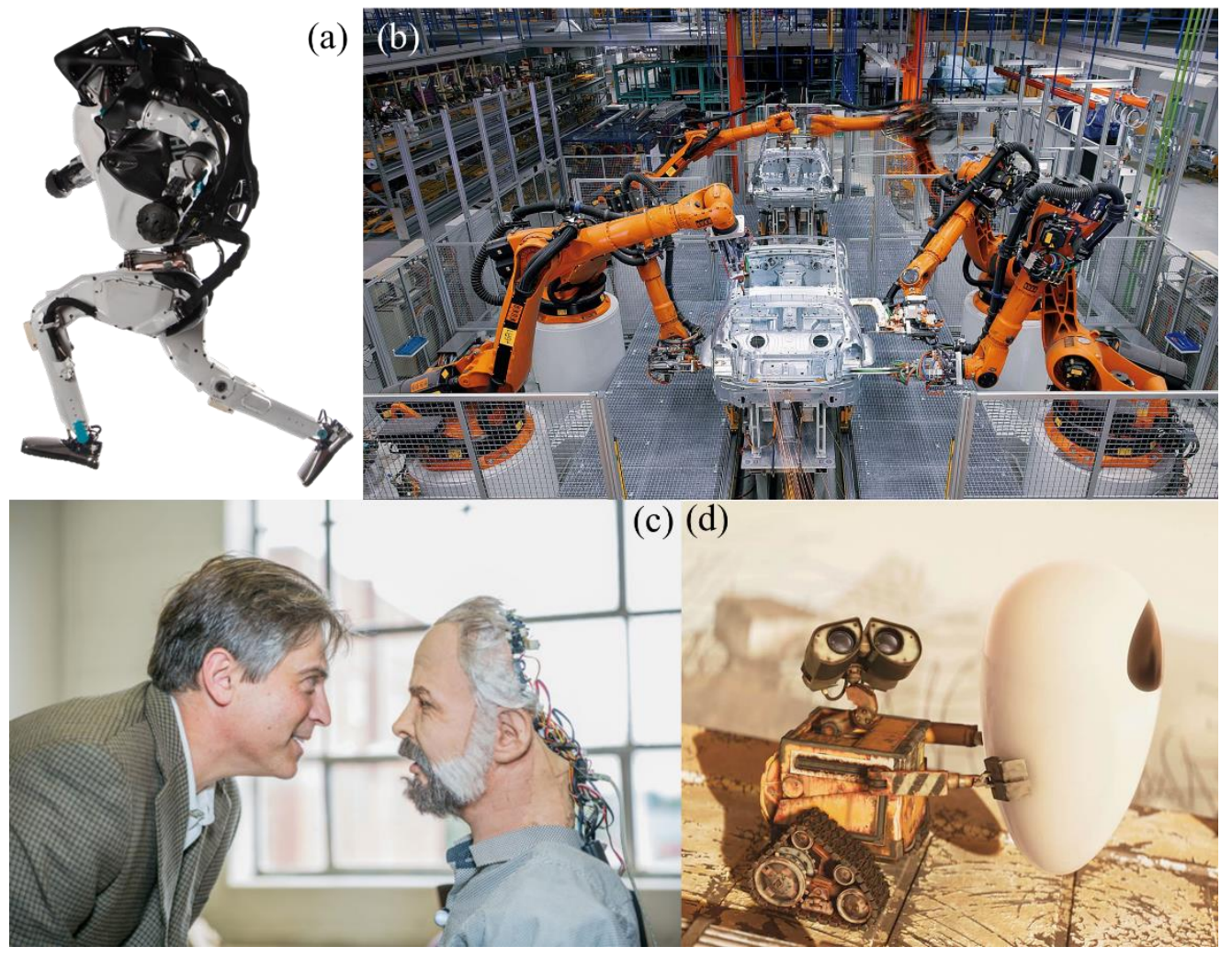

Figure 1-2 Various types of "regular-sized" robots. (a) ATLAS [22]. (b) Kuka robots in a car assembly line [23]. (c) Human-like robot developed by Hanson Robotics, featuring Philip K. Dick [24]. (d) Wall-E, an iconic fictional robot figure from Disney movie [25].

On the contrary, a microrobot may not be constructed the same way as their conventional macro-scale relatives since their bodies are significantly smaller. According to the microrobotics community's understanding, the characteristic dimension of a microrobot is typically less than $1 \mathrm{~mm}$; or it is capable of handling micro-scale components and processes. The limited size reduces integration level, so that not all elements can be integrated into the body. In extreme cases, such as the bubble robot and magnetic-fielddriven robots depicted in Figure 1-3, the precisely fabricated microrobot body itself is part of the actuation mechanism. While all the rest of sensing, source of power, and reasoning are built externally to support the tiny body. 


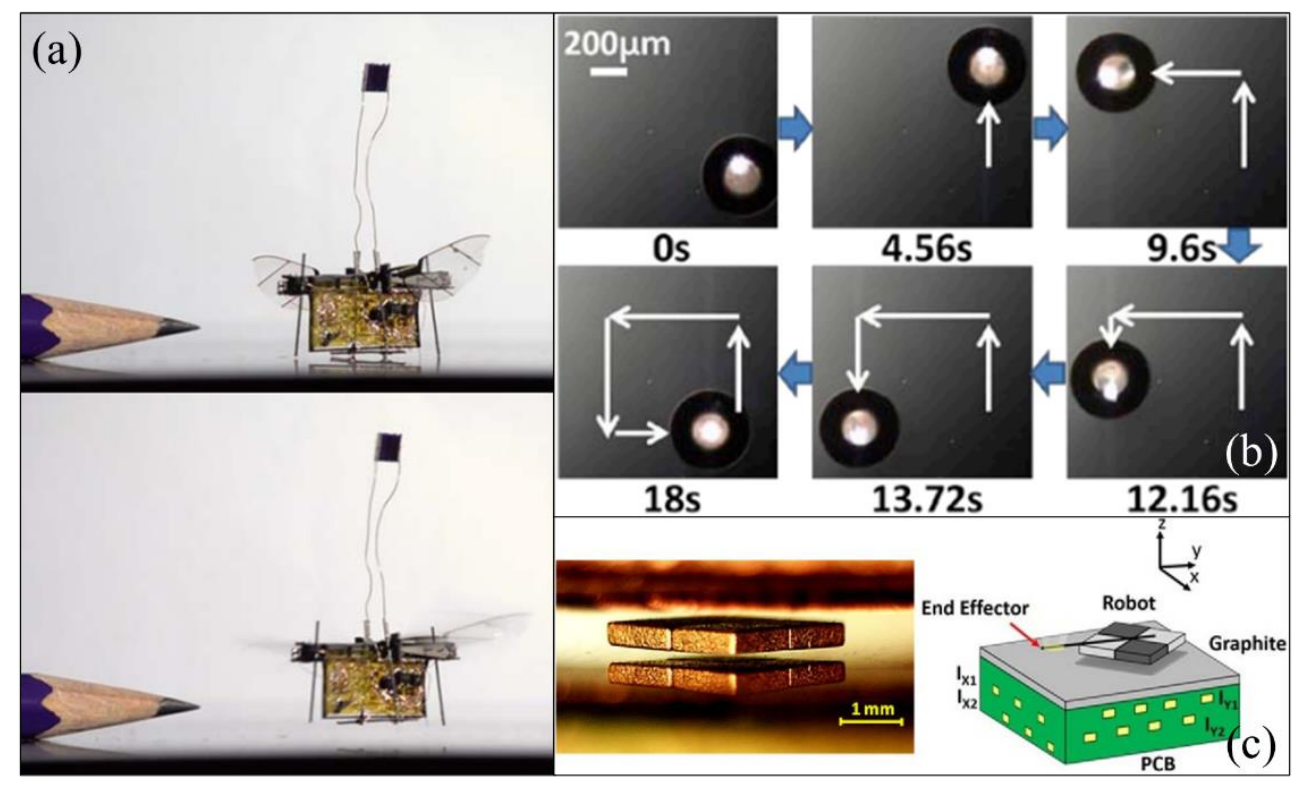

Figure 1-3 Microrobot examples. (a) Piezoresistive type, RoboFly [26]. (b) Bubble type [27]. (c) Magnetic type [28].

Figure 1-3 demonstrates three microrobots that having different integration level. The RoboFly [26] microrobot shown in (a) has the highest integration level, includes power reception, actuation, control, and sensing. (c) is much simpler in both fabrication and functionality, the body is a magnet piece with a needle. (b) is the simplest of all three, the body is merely a size-controlled bubble in liquid.

\subsubsection{Silicon MEMS-based microrobots}

Silicon's favorable mechanical properties and well-developed fabrication processes made it a good choice for MEMS technologies. For example, deep reactive ion etching (DRIE) technique can be used to shape a Silicon piece reliably and rapidly. Metal deposition processes can be used to change mechanical stress and further adding curvature into the body. Chemical vapor deposition (CVD) process can deposit Silicon to create overall 3-dimensional structures. A decent elasticity and thermal expansion coefficient of 
Silicon makes it a good thermal actuator, which produces output force in milli-Newton level [29].

Doped silicon is also a piezoresistive material with gauge factor as high as 200, 100 times over metal foil gauges [30]. This property provides the possibility of embedded deformation sensing option by detecting resistance change. Besides, capacitance detection is another well-developed technique can be used as a sensing method to provide location feedback information.

\subsubsection{Energy Source of Microrobots}

Microrobots can be actuated in multiple ways, magnetic field is one of the most popular. This method requires the microrobot fabricated by or including magnetic materials. The motion of the microrobot is driven by altering the magnetic field in the space, such field can be precisely generated by electromagnetic coils. By manipulating the position of those coils, the microrobots can be controlled in 3-dimensions. Position feedback of this method rely on vision, however, if the microrobot experiences little motion resistance compare with the actuation force, position feedback may not need. However, those coils can be bulky, and it is less straight-forward to individually and simultaneously manipulate more than 1 microrobot. Examples of such systems are demonstrated in Figure 1-4.

Thermal actuators are driven by temperature difference, either through current or other means. Thermal expansion of the material introduce deformation, which can be utilized to create locomotion. In this case, the temperature difference between the actuator and the surrounding environment decides the motion amplitude. In ambient, the air may sink the temperature raise, and introduce fluctuation in the motion. 


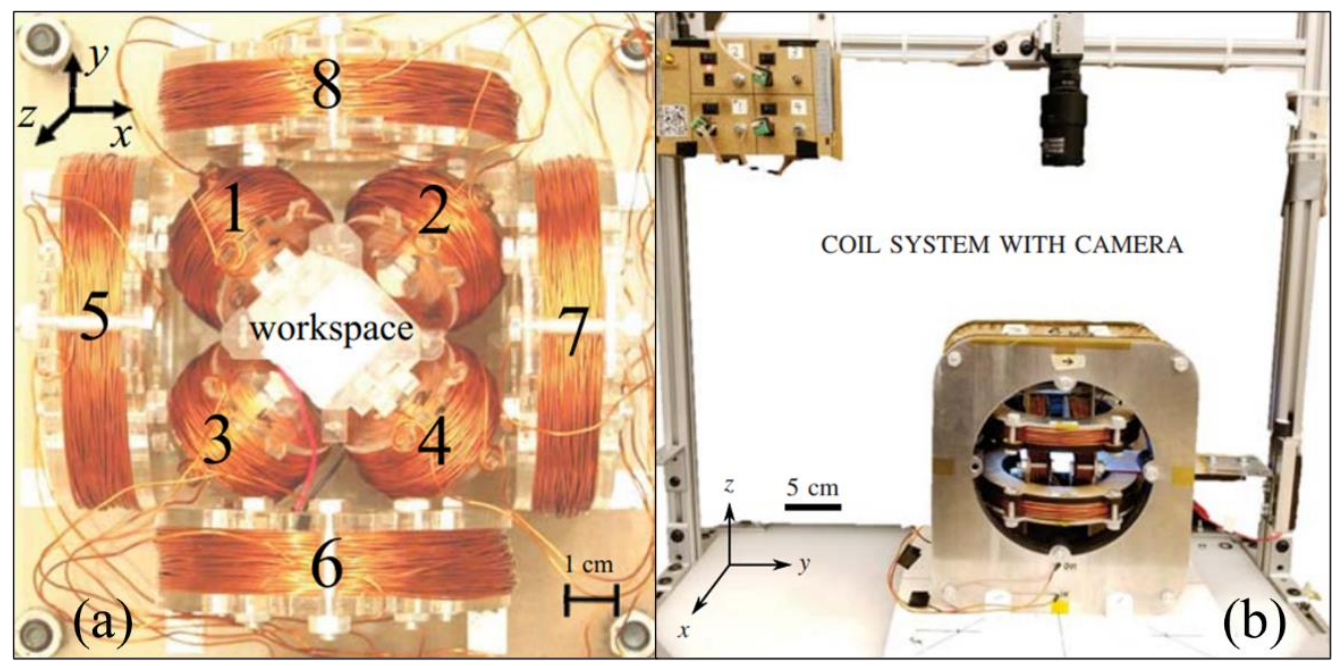

Figure 1-4 Examples of Electro-magnetic coils used in controlling the magnetic-based microrobots. (a) Eight coils used to enable 8 degrees of freedom (DOF) on magnetic microrobot [31]. (b) A system used to control two individual magnetic-driven microrobots simultaneously [32].

Energy can be harvested by solar cells, the multijunction concentrator type can even reach $40 \%$ efficiency, which makes powering microrobot with artificial light source possible. Meanwhile, the emerging Li-Fi technology enables data transmission by ambient light. Combine data communication with wireless power harvesting enables microrobot operate untethered.

Many materials accumulate electrical charge under mechanical stress, vice versa, stress is generated when electrical field is applied. This effect is also widely used in microrobot's actuation. High force output, high energy conversion efficiency, and high actuation frequency can be achieved on these actuators. To actuate them, a high voltage source is necessary.

\subsection{Challenges}

Constructing microrobots involves numerous challenges. Specifically, the gravity's dominance gives way to other micro scale forces and effects, such as the Van der Waals 
force, electrostatic force and surface tension [33]. These effects cause micro and nano objects to be prone to stick together in an undesired way. Micrometer-size objects are difficult or impossible to see with the naked eye, thus there is a need to use cameras and microscopic lenses to observe their operation, which adds complexity to manipulation and control. Due to the size limitation, microrobots cannot contain all conventional mechanical structures and electronics in macro-scale robots; as a result, processing, sensing and motion capability must be incorporated in a different manner. Eventually, micro and nano scale manipulation is more likely affected by environment fluctuation, such as system vibration, electrical noise and even dust particles. These challenges are discussed in more details below.

Microrobots distinguish themselves from conventional robots by their small size, although it is not necessarily limited in micro-meter range. The largest microrobots widely accepted are measured in several centimeter size, examples of which include the aerial microrobot RoboBee [34] [35] [36] [37] and its parallel development RoboFly [38] [39] [26] [40], and the crawler type microrobot ARRIPede [41] [42] [43]. For such spacelimited designs, although electronics are included in their body, challenges like weight reduction, wireless control and power delivery, and method of actuation must be reconsidered. Meanwhile, most of mobile microrobots are measured in micrometers, such as magnetic field driven [44] [45] [46] [47]. They face a different set of problems, such as vision feedback, sensing, environment fluctuation, and friction forces from the environment.

The construction difference between a conventional and a microrobot should be the most prominent distinction. In a conventional construction, the robot should contain all 
processing power such as a main computer, sensors such as a camera, and actuators such as motors within the robot itself. However, due to fabrication limitations, it is impossible to include all above elements in a microrobot design, neither the motor nor the camera is small enough to fit. Instead, their body can only contain a minimum number of elements, while heavily relying on external equipment to provide the rest of the features. Most of the time, compromises must be made so that the microrobot itself only contains a fundamental actuation element. In the case of the magnetic-driven microrobots, it is quite often that the microrobot is simplified into a single magnet [48] [49] [50] [51]. This challenge means the researchers must rely on different physical phenomenon to develop the functionality of the microrobot. Meanwhile, nanoscale manipulation will require a vacuum environment to ensure successful operation and scanning electron microscopy to supervise the process.

The powering and control in the microrobot domain are also different from conventional macro-scale robots and is often provided externally from an ambient field. For example, the bubble microrobot [27] [52] [53] is constructed totally in a liquid environment, while the power and control are provided by an external focused laser. This aspect imposes the challenge of tracking the robot with a laser beam, while system vibrations introduced in the tracking motion should be minimized.

Feedback is needed for microrobots to achieve precise control of their positions. Vision feedback through microscopes or the SEM is very popular since it is difficult to integrate other conventional sensors and supported circuitry into most microrobots. However, vision feedback suffers from limitations in illumination, occlusion, and processing speed, and control methods that are robust and scalable are the subject of on-going research in the scientific community. 


\subsection{Contributions}

In this thesis, the concept of microfactory is being advanced by the introduction of three types of microrobots, innovations described below:

1) The ChevBot is the first laser-driven microrobot that operates in dry environments. The microrobot has a size under $1 \mathrm{~mm}$ has multiple "feet", an assembled dimple contacting with the operating surface, and locomotes using stick and slip principle under laser power from a single $532 \mathrm{~nm}$ green laser beam. It can achieve 20 to $100 \mu \mathrm{m} / \mathrm{s}$ velocities under different irradiation conditions. The robot design includes a Chevron-style opto-thermal MEMS actuator that makes it possible to control the robot velocity via its laser beam pulse frequency. Such concept was proposed by [54] but never experimentally validated. We introduce multi-physics models to predict the ChevBot's behavior prior to fabrication, proposed a fabrication process for realizing physical prototypes, and demonstrated its operation under experimental conditions.

ChevBot is the first microrobot of its type, that uses laser directly to induce energy into MEMS thermal actuator and hence generates locomotion on a dry operating surface. By doing so, it achieves fully untethered control and power. It also experimentally proved the MEMS thermal actuator can be driven by a pulsed laser, and the locomotion speed can by controlled by the pulsed laser parameters. ChevBot demonstrates laser as a power source, can be utilized to generate locomotion of a microrobot without firstly convert into electricity [26], or rely on optical trapping [52]. It also provides another actuating mechanism other than the magnetic/electrical field, that can be used to manipulate micro and nano objects that are magnetized. 
2) The sAFAM, or Solid Articulated Four Axes Microrobot (sAFAM) is a stationary, world's smallest millimeter size robotic arm nano-positioner with 4 DOF, constructed from MEMS building blocks by microassembly techniques. Our work provided the theoretical analysis of its performance, and fabrication processes for its physical realization. Once samples were produced in our lab, the microrobot was experimentally investigated and shown that it can position a tip in a workspace of approximately $16 \mu \mathrm{m} \times 20 \mu \mathrm{m} \times 118 \mu \mathrm{m}$ with $20 \mathrm{~nm}$ resolution and repeatability.

The micro-assembled and MEMS-based sAFAM demonstrates a new type of nano manipulator that significantly smaller than their conventional counterparts that driven by piezo actuators and electrical motors. The most prominent advantage of the reduced size enables the possibility of in-situ, cooperative manipulation within most SEM chambers. The improvement also made in-situ SEM semiconductor testing feasible under current setup.

3) The SolarPede is a centimeter-size crawler microrobot, powered by artificial light and can be wirelessly controlled. It is an attempt to integrate a mobile MEMS base/legs with an electrical backpack and solar cell batteries. The MEMS thermal actuators were arranged in a central-symmetric pattern that enables omni-directional motions, in which other counterparts are mostly holonomic [42] [55] [56]. Due to the mechanical property, the motion precision of SolarPede can be expected in nano meter level.

We contributed finite element and lumped-parameter models predicting its operation on a flat dry substrate. The power-balance, fabrication and integration processes were demonstrated using several completed prototypes. Finally, omni-directional motion in 3 DOF with more than $10 \mu \mathrm{m} / \mathrm{s}$ velocities have been demonstrated with a microrobot 
prototype configured as a conveyor, which experimentally proofs that the energy from artificial light can be collected by solar cells and further used to actuate MEMS thermal actuator based microrobots.

Figure 1-5 shows a 3D rendering of our microfactory concept, featuring ChevBot, sAFAM and SolarPede. The sAFAMs are at fixed positions in the factory and several of them can form a basic microassembly site with pick-and-place or probe capabilities; the SolarPedes move large distances between different assembly sites to transfer raw materials, or rotate within one assembly site to assist the assembly process. They can also be used as conveyors if operated in "belly-up" mode. Finally, the ChevBots are located on the workspace of the SolarPede, where they are used as precise material/part carriers, or nanomanipulators by pushing with a compliant force-sensitive end-effector.

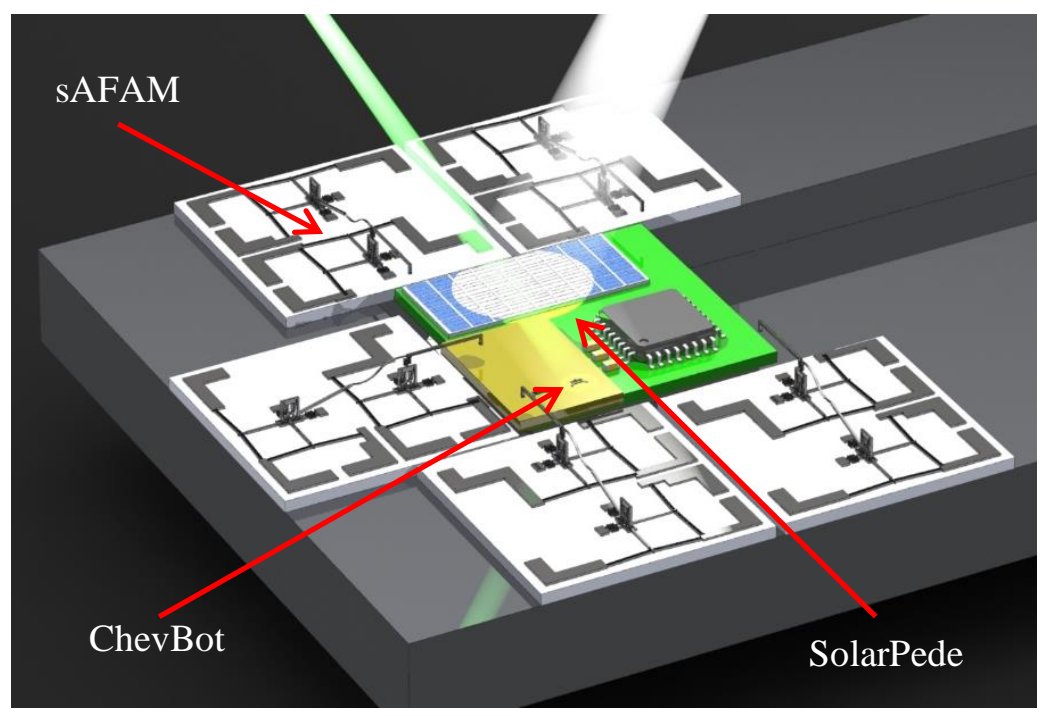

Figure 1-5 3D rendering of proposed microfactory, featuring ChevBot, sAFAM and SolarPede.

In the course of research, several journal and conference publications have been published and presented at international meetings, and some have received best paper awards as indicated below. The work and publications were supported by funding from two National Science Foundation grants, \#1734383, NRI: FND: Light-Powered 
Microrobots for Future Microfactories, 2017-2021, and \# 1849213, RII Track-1: Kentucky Advanced Manufacturing Partnership for Enhanced Robotics and Structures, 2019-2024. The list of publications is listed in Appendix E.

\subsection{Thesis Organization}

This thesis is organized as follows: Chapter 1 and 2 discuss related scientific literature on microrobots and microfactories, to highlight the contributions of this thesis; Chapter 3 gives detailed information about the ChevBot, including analysis, design, fabrication, and experimental characterization; Chapter 4 and 5 discusses the sAFAM and SolarPede microrobots, respectively, including their analysis, design, fabrication and experimental characterization; Chapter 6 concludes the thesis and discusses future directions for research. The Appendices provides more detailed technical information, such as Silicon on Insulator (SOI) wafer processing recipes, ChevBot's tracking experiment technical setup, and SolarPede's Printed Circuit Board (PCB) design and embedded microcontroller code. 


\section{CHAPTER 2 BACKGROUND}

\subsection{Microrobots}

Autonomous microrobots have been investigated by many in the last few decades, for applications in micro and nano manufacturing, biology, surgery and in-body exploration. Based on construction complexity, microrobots can be classified into those incorporating all necessary power, control and sensor infrastructure onboard [36] [57] [58] [59] and those that harvest energy from the environment and are controlled by reacting to external stimulus signals [60] [61] [62] [27] [63]. If we look into the problem further, one can see that among the three factors of power, control, and actuation, the power delivery limits microrobots development the most, since the energy density of an energy storing device has a positive correlation with its volume, in the order of 3 . From this perspective, microrobotics systems can be categorized into two: self-powered with energy harvesting (type E) and remote-powered (type R). The type E microrobots carries energy storing device such as Li-Po battery, which allows the microrobot to power on-board sensors and controllers needed for interaction with the environment, reasoning and even reacting to it, so that it is similar to conventional macroscopic robots. However, type $\mathrm{R}$ microrobot is fundamentally different; the lack of the energy storage device on board means that control, sensing, and computing (reasoning) have to be realized externally (outside the robot's body, such as the electromagnetic coils used in magnetic field driven microrobots). Robot itself is a passive device, like a puppet, reacting to the external physical stimuli. 
For liquid environments, magnetic multi-DOF steering has gained wide acceptance due to biological compatibility and performance [61]. For applications in dry environments, such as in microfactory for nanotechnology, an important subclass of microrobots are those fabricated using MEMS technology on silicon substrate. Regardless the fabrication methods, magnetic, thermal, electrostatic, laser, focused light and piezoelectric effects are all widely used in microrobot actuation for this application. In [59], the authors compared six most popular actuation methods in recent research. The MEMS electrostatic actuator derives its popularity by very low power consumption and reasonable amount of force output, and these two merits allow many of those designs to realize untethered operation. Donald et al. [60] demonstrated a MEMS microrobot design driven by electrostatic force on an engineered surface. Thermal actuation is also widely applied on microrobots, often by bimorph or Chevron actuators [61] [54] [64]. Thermal actuators provide high force output while consuming highest power among other mechanisms, thus many designs are tethered due to the need to provide power. There was also significant advancement in the studies of untethered magnetically powered microrobots [65] [66] [67]. These types of robots can operate in dry or wet environments, reach relatively high velocities, move on a variety of surfaces [66] [67], interact with objects [67], and provide excellent motion control. However, systems with magnetically powered robots include external coils of significant size for magnetic field generation. Some of the designs require significant magnetic fields for operation (1-30 mT) [65] [67], and the devices have to be fabricated from the ferromagnetic material. These factors could be potentially limiting in microfactory applications. 
A key limiting factor in advancing microrobot technologies with non-magnetic drives is the delivery of power. Many microrobots have been experimentally demonstrated using tethered power [36], because the reported energy harvesters of that size can only provide power below $\mu \mathrm{W}$ range. In the case of thermal actuators utilizing bimorph or Chevron structures [59] [54] [68] [43], these provide high force output but have to be tethered due to their power requirements. Direct wireless power delivery to miniature robots has several inherent advantages over energy harvested and stored on-board. Under this paradigm, the power required for the operation of the device is externally provided by a dedicated source. The source can also control and communicate with the robot, and this greatly simplifies the internal structure of the microrobot.

\subsection{1 the ChevBot}

The use of light or laser power in microrobotics is motivated by the availability of lasers with high energy concentrations and high directionality. However, its uses have been focused on relatively large dimensional scales (a few $\mathrm{mm}$ ) and very small scales (below 10 $\mathrm{nm})$. At the millimeter dimensional scale, recent examples of optical actuation include the Robofly [26] in which a concentrated laser light beam provides power to a photovoltaic cell then powers actuators and on-board electronics. Optical trapping is another example of actuation using light energy, but in this case very small forces, $\mathrm{pN}$ level, are generated to actuate objects smaller than 10 microns in aqueous media. And thermally driven impingement has also been exploited at the sub-millimeter scale in dry environments for sub-millimetric flight [62] and in liquid media to manipulate biological cells using optically actuated bubbles [27]. 
The ChevBot is a type E microrobot. Its design [69] [70] is proposed by using the insights gained during modeling, and experimentation in [54] [69], which demonstrates stable directional locomotion, maneuverability, and achieving controllability in the future work. Furthermore, an automated laser-robot tracking system was designed and implemented using visual servoing in order to ensure continuous powered operation for the microrobot and record gating trajectories. Results indicate that the ChevBot generates straight trajectories with speeds in excess of $100 \mu \mathrm{m} / \mathrm{s}$.

\subsection{2 the SAFAM}

By combining microassembly techniques with silicon parts fabricated by standard CMOS processes, the complexity of the resulting microrobots can be significantly higher than before [43] [41]. Tsui [71] and Geisberger [72] invented a passive snap fastener style MEMS coupling device, widely known as the Zyvex connector, which greatly reduces the effort to perform microassembly tasks by accommodating relatively large micromachining and micro manipulating errors.

The solid Articulated Four Axes Microrobot [73] [74] is a newly articulated and assembled microrobot, falling under type R category. In our robot design, the arm assembly consists only of a monolithic micromachined silicon piece with an elastic leaf spring representing a motion coupling principle; and all parts are fabricated by surface micromachining technology. The sAFAM design was first examined using Finite Element Analysis (FEA) simulation, which was validated against experiments to confirm the anticipated workspace, then laser displacement sensor was used to evaluate the resolution and repeatability. Results confirm that the microrobot has a 3D workspace of approximately $16 \mu \mathrm{m} \times 20 \mu \mathrm{m} \times 118 \mu \mathrm{m}$, a resolution and repeatability below $20 \mathrm{~nm}$ in 
certain regions of its workspace, and a vertical force output of $45 \mu \mathrm{N}$, making it suitable for future nanoscale work. For instance, with an atomic force microscope (AFM) probe attached to the end-effector, sAFAM can be used as a low-cost precision manipulator or measurement instrument at the nanoscale, such as in studies related to cell adhesion [75], on thin-film coating friction [76], and carbon nanotube adhesion [77].

\subsection{3 the SolarPede}

Recent research has explored the possibilities of constructing microfactories that are affordable, flexible, and scalable. One solution towards such a goal often incorporates offthe-shelf motorized stages, actuators, and customized metal parts to achieve high precision processing and manufacturing [78] [79] [80]. However, these examples have several drawbacks, such as lack of parallel processing capability [80] and difficulty in scaling to lower dimensions [81]. Another solution proposed for next generation microfactories envisioned microrobots, both mobile and fixed [82] [28] [83]. Mobile microrobots will be needed to reposition material in the microfactory while being capable of wireless navigation and autonomous task execution [84]. Recent results in creating mobile microrobots for dry environments include cilia-like gated crawlers [85] [42], magnetic levitation [28] [86], piezoresistive stick-and-slip effects [87], and light-powered PZT actuators [88] [37].

The ARRIPede [85] [41] was an untethered, micro-assembled MEMS microrobot, capable of stick-and-slip operation while powered by an on-board Lithium-Polymer battery. While storing energy on-board for use during operation provides maximum functional flexibility, it is technically challenging to implement due to the unfavorable dimensional scaling of battery power sources. Such arrangement also requires the battery to be 
disassembled from the microrobot and recharged. Therefore, it is desirable to harvest ambient energy, for instance from light sources such that an onboard battery is not required. The ARRIPede's gait and locomotion methods have been well characterized and accompanied by stick-and-slip dynamic models [43] [41]. This early design demonstrated a great payload carrying capacity of $9 \mathrm{~g}$, and non-holonomic mobility characterized by forward only and large turn radii with speeds in excess of $1 \mathrm{~mm} / \mathrm{s}$. However, the operation of this microrobot was seriously limited due to a short battery life (approximately 10 minutes), a nonholonomic motion dexterity on the operating surface, and the lack of wireless communication for start, stop and feedback control.

We design and experimentally validate SolarPede, a solar-powered micro-crawler, that overcomes many of the operational limitations of ARRIPede. Unlike ARRIPede, the SolarPede does not use a battery to store energy in order to reduce weight and complexity of the system, and it was designed to operate in a controlled environment with artificial light that can constantly track its solar cells and provide perpetual power. Microrobot actuation of the SolarPede was accomplished via electrothermal "Chevron" actuator banks, referred to as "thermal actuators" in this thesis, and vertically micro-assembled legs, realized with snap-fasteners [72]. On SolarPede, the actuators and legs were rearranged in a differential drive configuration to achieve omni-directional, rather than nonholonomic locomotion. An on-board battery is no longer required for SolarPede and was replaced with high-efficiency solar cells to eliminate the need for battery recharging, while balancing microrobot power with solar energy from a solar simulator. The SolarPede's on-board computer is a Bluetooth-enabled 32-bit microcontroller unit, which increases both programming and wireless communication flexibility. Electronic backpack prototypes 
implementing solar-power harvesting and gait sequencing control were implemented and experimentally tested. By integrating all subsystems of the SolarPede, we demonstrated a fully untethered, light-driven omnidirectional microrobot operating as a nano positioner on planar surfaces.

A dynamic model was proposed and experimentally validated to predict the microrobot behavior. This model was used as a design tool and to understand the motion characteristics of the microrobot. The scientific contribution of our work consists of the novel leg and gait designs to achieve omni-directional motion, as well as the methods employed to accomplish microrobot energy balance through leg power-multiplexing, solar energy harvesting, and electronic backpack design.

In contrast to the I-Swarm light-driven microrobots [87], SolarPede's design and form factor allow it to have a much larger payload capacity, utilize off-the-shelf electronic components, and achieve nano-scale precision for operation inside a microfactory. Specifically, the measured weight of the SolarPede is $4 \mathrm{~g}$, while its vertical legs are capable of supporting 9-gram payloads [41]. The microrobot's thermal actuators are capable of generating large forces in excess of $50 \mathrm{mN}$ [29] and achieve nanometric motion resolution [42]. As a result, the SolarPede specifications are closer to a Scanning Electron Microscope mobile microrobot such as MINIMAN [89]. In this thesis, we report on results obtained to date validating that our concept is sound, including controlled operation of our microrobot in "belly-up" conveyor mode. Results suggest that in the near future, a mobile SolarPede can be miniaturized to a $\mathrm{cm}$-scale form factor for crawling operation required in microfactory tasks. 


\subsection{Microfactory}

In the conventional manufacturing environment such as the assembly lines, robots are already widely used and have been studied for decades. Each of them on the assembly line is designated for one single purpose to achieve the assembly of the final product. Such streamline manufacturing method produces most of the items we use every day. For example, on car assembly lines, robots are used to assemble doors onto frames or spray paint. In a PCB fabrication house, electrical components are picked up and placed by Selective Compliance Articulated Robot Arm (SCARA). The robots used in such cases are optimized to better fit the product assembly goals; these optimizations including operating under appropriate workspace, able to generate precise motion and force output, and adequate DOFs. Naturally, if the assembly goal has been changed, the tools used in the new scenario should also be reconsidered accordingly.

To perform micro and nano scale manufacturing using conventional tools is particularly challenging and expensive. For example, self-assembly technology provides possibility of mass production of nano scale objects. Once the self-assembly is done, it is difficult and expensive to build more sophisticated products with macro scale machines, due to the fact that conventional machines such as robot arms and motorized stages are much larger than the micro and nano scale objects. The inherent manufacturing error of those machines accumulates thus makes their accuracy, repeatability, and resolution inadequate for such tasks. Meanwhile, the micro and nano manipulation tasks often require restrict

environmental control such as clean room environment. However, because the conventional machines often occupy larger space comparing with microrobots, it is less 
space efficient to use them under such environment. This in turn caps the parallel processing capability, further increases cost.

The concept of a microfactory was proposed to fulfill such goal. Similar to a normal factory, a microfactory utilizes multi-functional, miniaturized robots that operate within a small and controlled environment to accomplish assembly tasks [90]. That means the tools used are of a comparable size with the processing objects. If the miniaturized robots are precisely made, such model can achieve high precision [78], resolution [81] and repeatability [91] in micro manipulation. The cost to produce such miniaturized factory setup should decrease so that parallel processing capability can be achieved with lower cost [81] [92]. With the smaller size, multiple units can be easily contained in a controlled environment, such as a desktop cleanroom [93]. Besides size difference, conventional electrical motors and actuators will be replaced so that vibration can be reduced [90]. Similar to a normal factory, the microfactory should be modular design to allow change of tools [94] [95] [96], and eventually, the microfactory is more power-efficient [97].

Many efforts have been directed into the development of a practical microfactory. In 1997, N. Kawahara et. al. [98] envisioned and discussed different approaches to accomplish the microfactory. The study classified the microfactory into two major categories, "Fabrication by Desktop Factory" and "Fabrication by Small Robots." The first category, "Fabrication by Desktop Factory," resembles a miniaturized conventional factory setup, which provides a similar workflow of an assembly line. Tools like precision positioning units, micromachining units, vision units, and conveyor units are widely used and appear in different sizes and shapes [99]. Many of the microfactories we see today are under "Fabrication by Desktop Factory" category [81] [95] [92] [97] [94]. In the second 
category of "Fabrication by Small Robots," a swamp of microrobots are used to accomplish assembly and manipulation tasks in a cooperative way.

Z.Zhakypov et. al. [81] [79] [100] pursued the first category. In their study, two delta robots, a laser processing tool, an auto focus camera, and a rotational conveyor platform were used within an environmentally controlled encapsulation, and they have demonstrated serial manipulation of sub-millimeter accuracy. A modular microfactory developed by Verettas et. al. [93] is a highly reconfigurable system, each module (microbox) contains fundamental elements of robotic mechanism, cleanroom workspace, and conveyors. Multiple microboxes can be used in a serial way that are similar to an assembly line except much smaller. Diederichs et. al. [80] prototyped their microfactory design that features high precision (below 100nm) and ease of control. In their design, a robotic arm hangs over the motorized precision $x-y$ stage, the end-effector is interchangeable - providing the option of a gripper or vacuum tip - and the whole factory can be tele-controlled with either a gamepad or haptic device.

Although all above efforts were directed towards miniaturizing the size of a microfactory and improving precision measurements, they all used conventional tools, but on a highly customized and much smaller scale. In order to push the level of autonomy, efficiency of parallel processing, and scale of manipulation, the Next Generation System (NGS) group pursues the second method of "Fabrication by Small Robots" to build the microfactory. In our research, three different microrobots with various functionalities were analyzed, fabricated, and prototyped. 


\section{ChaPter 3 THE LASER Driven Microrobot - ChevBot}

\subsection{Microrobot Design}

ChevBot is an assembled MEMS microrobot from Silicon components and is driven by a collimated laser beam approximately $800 \mu \mathrm{m}$ in diameter. The early concept of such microrobot was discussed by M. Pac et. al. in 2011 [54], they have articulated the feasibility of the microrobot mathematically and proposed steering paradigm but never experimentally validate it. In our research, we came up with our own unique solution and created the ChevBot microrobot. We have proposed multiple designs with modified dimension parameters, two of the variations are shown in Figure 3-1. Both of them have 6 pairs of chevron beams, each approximately $5 \mu \mathrm{m} \times 200 \mu \mathrm{m} \times 20 \mu \mathrm{m}$ in size.

Among the two shown in Figure 3-1, (a) was an early prototype that was used to proof both microassembly and laser induced motion on the operating surface. Based on (a), we made structural modifications so that the locomotion of the ChevBot tends to follow a straight line, shown in (b). Regardless, they share many design similarities, such as assembly process, the thermal actuator design, and gaiting method.

The ChevBot design studied in this thesis, shown in Figure 3-1 (b), has four major parts: two "feet" extending from the body frame, each forming a $135^{\circ}$ angle with adjacent body; the thermal actuator and its shuttle "anchored" within the body frame, and a dimple

attached to the assembly pad of the shuttle. The microrobot measures $734 \mu \mathrm{m}$ in length and $427 \mu \mathrm{m}$ in width, and with an assembled dimple, its height is approximately $40 \mu \mathrm{m}$. Each 
beam in the thermal actuator is $5 \mu \mathrm{m}$ wide and they form an acute angle of approximately $87^{\circ}$ with the body frame.

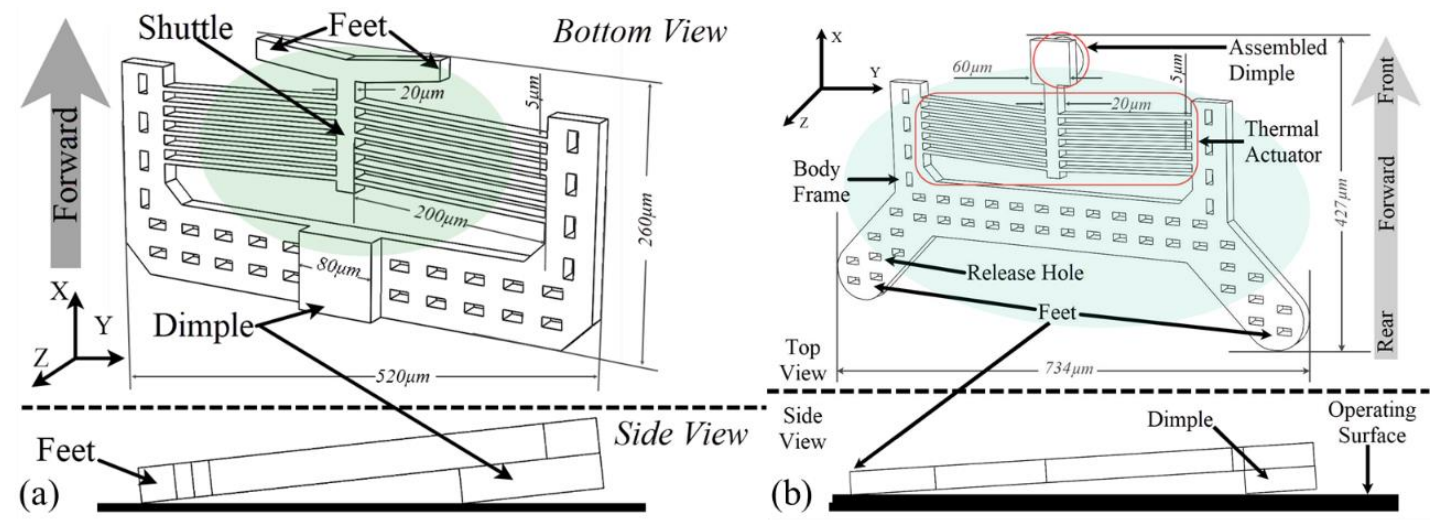

Figure 3-1 3D drawing of ChevBot with critical dimensions. (a) An early prototype [54]. (b) ChevBot validation design. Consistent with locomotion direction, the assembled dimple is defined as the front of the robot, and the feet are defined as rear of the microrobot.

After the dimple is assembled, the ChevBot can lay on an operating surface at a small inclination angle, with the inner edge of the cylinder-shaped dimple and the feet as three contact points to the environment.

\subsubsection{Principle of Operation}

A stick-and-slip model was adopted to describe and predict the motion of the microrobot [54] [69]. The power and control signal generating the gait is provided by a collimated laser beam that covers the entire body of ChevBot; meanwhile, the pulse laser beam can be operated under both burst and continuous mode to initiate actuation. Pulsed laser operation was chosen in order to induce cooling-heating cycles of the ChevBot's body, which in turn cause cyclical motion of the dimple, due to the thermal expansion effects. To sustain motion, the laser coverage of the microrobot needs to be maintained, e.g. the laser beam must follow the motion of the microrobot. During the laser-on cycle, the temperature of the microrobot body rapidly increases and activates the thermal actuator, which causes 
the dimple to stick to the substrate and the feet to slip toward the dimple. During laser-off, the cooling cycle, the feet stick to the substrate and push the dimple forward to finish a full cycle of stick-and-slip motion, resulting a net forward displacement. Above process can be illustrated by Figure 3-2. The proposed paradigm is affected by several factors, for example: the tilting angle of the body, the weight distribution of the body mass, and the length of the microrobot. Chapter 3.4 discusses such factors in detail.

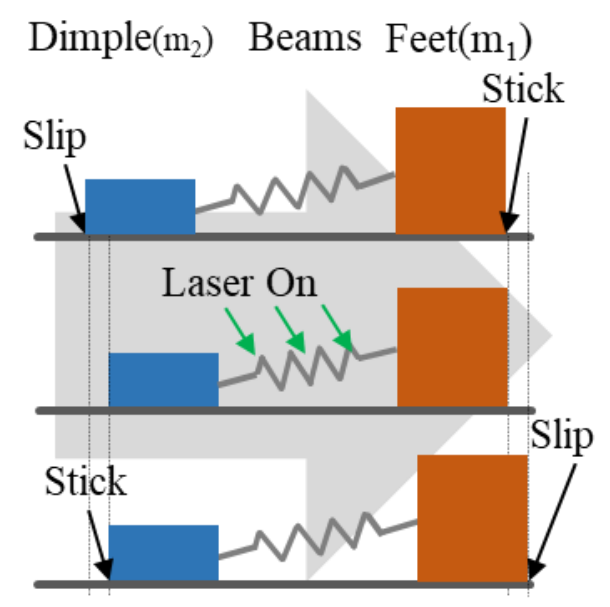

Figure 3-2 Illustration of ChevBot's stick-and-slip locomotion.

\subsubsection{Design Efforts Toward Straight Trajectory Locomotion}

The control of the Chevbot's motion depends on its geometry. Therefore, design and relative location of the chevron actuator, dimple, and legs need to be considered to enable locomotion on a flat surface in a controlled way. It is not immediately clear how many feet or dimples must be located at the front or rear of the microrobot. Below we discuss why the choice of design in Figure 3-1 (b) is likely to result in locomotion along straight trajectories.

In a simplified general case, the microrobot contacts the operating surface through dimples and feet, represented by squares in Figure 3-3. The circle represents the body/actuator of the microrobot, where most of its mass locates. The lines represent the 
"limbs" that connect the body with the feet. In a stick and slip gait, either the feet on the front or at the rear of the microrobot serve as the "driver", while the other pair keeps balance, acting as a "supporter".

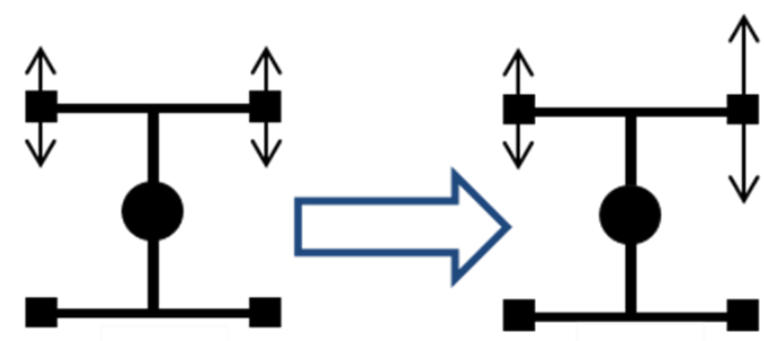

(a) (b)

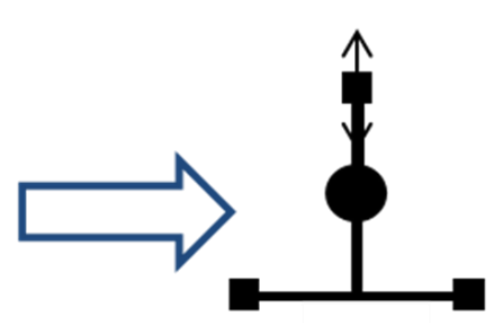

(c)

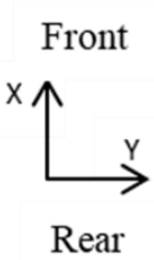

Rear

Figure 3-3 Abstraction of the ChevBot design depicting the body (circle) and feet (square). The arrows indicate the reciprocating direction of the feet motion when actuating force is (a) balanced; (b) imbalanced. (c) Single front contact to increase motion stability.

In the above schematic, the driver feet reciprocate back and forth, and produce friction/stiction contact with the substrate to generate locomotion. In an ideal case of the left and right driving forces are equal and aligned, the combined driving forces can be either pulling the microrobot forward to the $\mathrm{X}$ direction or pushing back towards - $\mathrm{X}$ direction, as shown in Figure 3-3 (a). While the forward resulting motion is stable, the backward motion is not, similarly to a front wheel/rear wheel automobile driving scenario. Furthermore, in practical scenarios, the feet experience imbalanced friction forces or net moments due to varying surface conditions and manufacturing imperfections, causing the microrobot to steer unpredictably. As a result, we can combine the two driving contacts into one to reduce and even remove the moment to maximize the chance the ChevBot gaits straight forward, as shown in Figure 3-3 (c). However, this phenomenon can be utilized in designing a steerable version of ChevBot. 
Therefore, to improve the straight locomotion stability of the ChevBot compared to the design in [69] we want to ensure that the front feet pulls the microrobot forward, rather than push it backward. This was accomplished by reducing the two front feet to a single "dimple" location and orienting the chevron direction of the micro actuator in the direction which causes desired forward locomotion, resulting in the ChevBot design shown in Figure 3-1 (b).

\subsection{Fabrication and Assembly}

The ChevBot was fabricated from a Silicon wafer using a standard SOI process, and further assembled with a custom microassembly in our lab, as described in this section.

\subsubsection{Fabrication}

The ChevBot was fabricated on a silicon on insulator (SOI) wafer with $20 \mu \mathrm{m}$ device layer thickness using a Deep Reactive Ion Etching (DRIE) process. The microrobot layout, its dimple and other assembly components are depicted in the mask design shown in Figure 3-4.

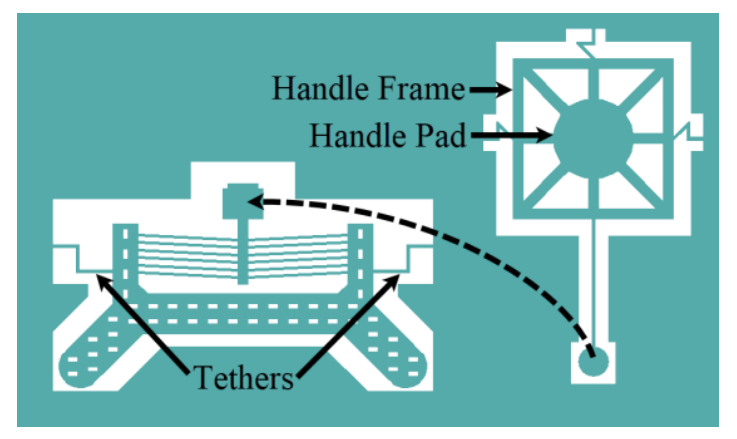

Figure 3-4 Mask layout of ChevBot body and its dimple assembly.

The major steps of fabrication process are discussed here, and the detailed recipe is provided in Appendix II. First, the wafer was first cleaned using the RCA process to remove any potential organic, oxide and iconic contamination. Second, photolithography 
was performed by spun photoresist Shipley 1827 on the dried wafer surface, and soft baked on a hot plate. An UV exposure was performed to transfer the patterns on the mask onto the photoresist. Then, the photoresist was developed in the designated developer to finish photolithography. Third, after inspection of the photoresist under microscope, the wafer was placed on the hot plate again to hard bake, so that the photoresist was hardened and ready to serve as a masking layer for DRIE.

The subsequent etching process carved the body of the microrobot out of the device layer of the SOI wafer. Preliminary etching time was empirically estimated, depend on the overall etching area and the etching rate of the machine for a given recipe. After etching for 20 minutes, the sample was removed from the chamber and the actual etching rate was estimated by dividing the measured trench depth with time. The photoresist mask along with process byproducts were removed after a successful DRIE process by oxygen plasma clean in a March RIE machine.

Another layer of photoresist was spun before dicing the wafer, so that the particles created during dicing would not fall into DRIE trenches and block the motion of the microrobot. The spin rate of this step is slow and does not require very hard photoresist. Finally, release and drying were performed after dicing. Individual dies were merged in $49 \% \mathrm{HF}$ acid for 10 minutes to release. After the 10 minutes period, all dies were removed from acid and rinse under deionized water in a plastic container to wash away HF acid. Drying was then automatically done using supercritical $\mathrm{CO}_{2}$ in a Critical Point Dryer tool.

\subsubsection{Assembly}

ChevBots and all other microrobots discussed in this dissertation were assembled by the custom NeXus microassembly station shown in Figure 3-5. To assemble the ChevBot, the 
NeXus microassembly station was configured to having two motorized manipulators $\mathrm{M}_{1}$ and $\mathrm{M}_{3}$, each targeting different set of manipulation tasks. The $\mathrm{M}_{1}$ manipulator holds the sample chuck, and contains two identical linear translation stages, stacked perpendicularly to each other at the bottom, and a rotation stage on top. The sample chuck is made of aluminum, with five vacuum-secured $1 \mathrm{~cm}$ by $1 \mathrm{~cm}$ die slots on top. Overall, the $\mathrm{M}_{1}$ manipulator fulfils $\mathrm{X}-\mathrm{Y}-\theta$ motion to the sample chuck. The $\mathrm{M}_{3}$ manipulator consists of a manual $\mathrm{Z}$ stage to adjust height, three motorized linear translational stages and one rotational stage stacked together to fulfil $\mathrm{X}-\mathrm{Y}-\mathrm{Z}-\theta$ motion for the end-effector. The rotational stage is mounted vertically on the $\mathrm{Z}$ stage, with a $3 \mathrm{D}$ printed fixture and an adapter, allow a vacuum tip end-effector installed horizontally. The dimple is picked up with the vacuum tip, moved to the desired location, and bonded to the assembly pad using UV epoxy.

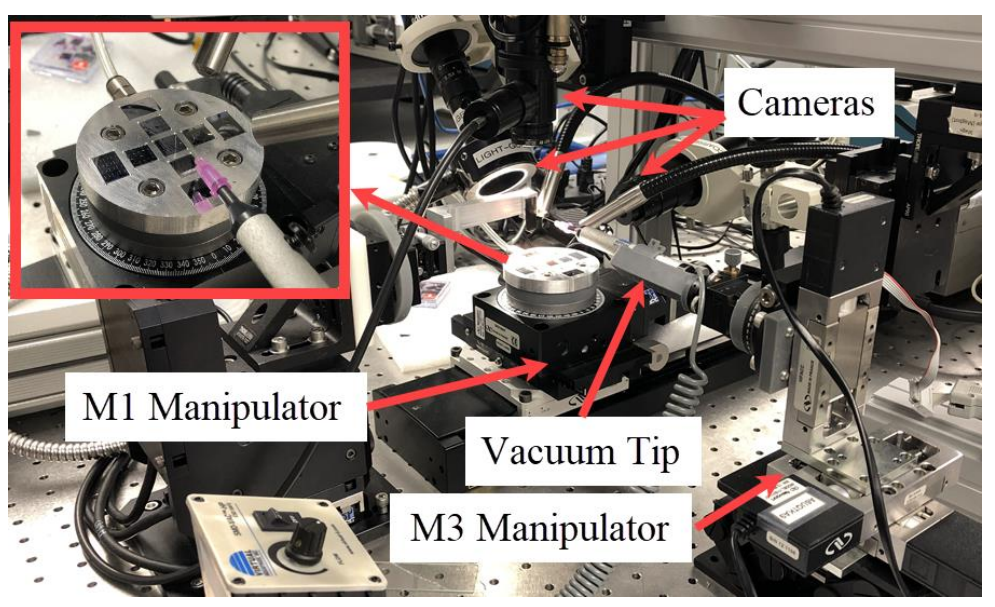

Figure 3-5 The NeXus microassembly station used to assemble ChevBots.

Assembly of the ChevBot aims to bond the dimple onto the front foot and flip the microrobot body so it forms a three-point contact with the operating surface. To simplify the assembly process, the ChevBot body and the dimple assembly were placed on the same die; before picking up the dimple, a small amount of UV adhesive (BONDIC ${ }^{8}$ L4G 3V5, 
Aurora, ON, Canada) was dissipated near the assembly site; then, with a probe tip, we broke the tethers that hold the dimple assembly, and the vacuum tip end-effector was aligned with the handle pad at the center. With the help of the handle frame, the operator slightly dipped the dimple in the pool of UV adhesive, then placed the dimple onto the assembly pad. Finally, a UV torch light was used to cure the adhesive in place.

\subsection{Experimental Setup}

In order to power and track ChevBot, we configured a custom experimental system as a combination of two sub-systems: an optical system for laser delivery and an automated visual tracking stage system to compensate microrobot's motion, so that ChevBot continuously stays under the laser beam. This system was used for two different experiments, one involving tethered microrobots to the substrate die, and another to study the motion of untethered microrobots on operating surface. In the case of the tethered ChevBot, only motion of the thermal actuator was measured. In the untethered case, the goal was to initiate and record stick and slip motion of the whole microrobot on a silicon substrate. A detailed technical discussion of the setup is provided in Appendix C.

\subsubsection{Optical System for Laser Delivery}

Laser irradiation tests with ChevBot were conducted using an experimental setup depicted in Figure 3-6. The main components of the optical testing system include:

- Explorer One Nd:YAG laser from Spectra-Physics, with 532nm wavelength, 2W Maximum power, $0.5-60 \mathrm{kHz}$ repetition rate and 10 to $40 \mathrm{~ns}$ pulse time width.

- A system of lenses, neutral density (ND) filters, beam splitters and mirrors to deliver the laser beam to the microrobot. 
- Four Newport X-Y positioning stages of 423 and 443 series, with two actuated by linear actuator TRA25CC and two controlled manually. They stack perpendicular to each other, while sample chuck was fixed on top.

- Tube lens, illuminator, beam splitter, National Instruments smart camera ISC1772C for automated tracking and Pixelink CMOS camera for visualization.

- A range sensor, LK-H008 from Keyence, to measure the displacement of the thermal actuator in tethered experiments.

During the experiments, the laser beam was passed through the neutral density filter and system of the lenses, toward the set of the adjustable mirrors, which directs laser light onto the ChevBot on the sample chuck. The last mirror is placed at angle, so that incident laser beam is at $20^{\circ}$ to $30^{\circ}$ angle to the normal of the arena's surface. As a result, the laser spot has an elliptical shape on the sample surface with a large waist diameter wdmax $=800 \mu \mathrm{m}$ and small waist diameter wdmin $=600 \mu \mathrm{m}$. The laser spot and ChevBot are aligned with help of the Pixelink CMOS camera, which is coupled with the NI smart camera by a beam splitter, so that both share the same field of view of the sample on the stage chuck. Both cameras and beam splitter are attached to the tube lens that is placed above the sample chuck. The laser module can be operated under either continuous or burst mode. Continuous mode generates a series of pulses, while under burst mode, user can specify the number of pulses within a burst and delay between each burst. For both cases, repetition rate of the fundamental pulses and diode current are adjustable. Thus, the power of the laser is affected by diode current and number of pulses within each burst. 


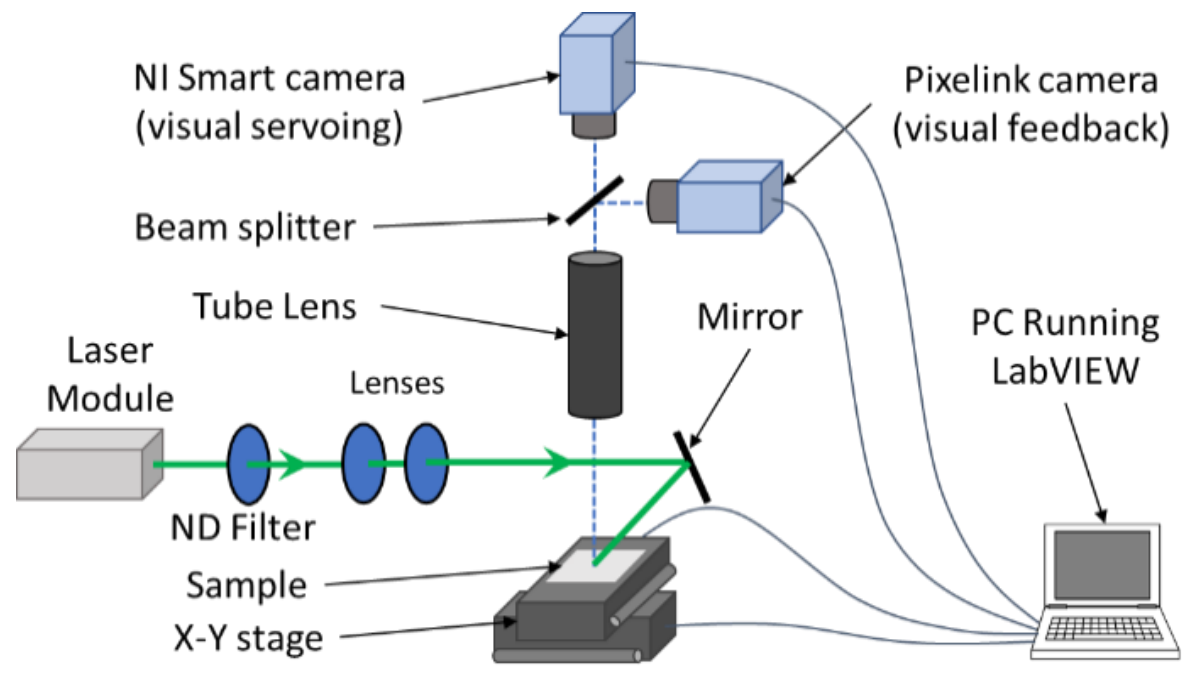

Figure 3-6 Schematic of laser delivery and vision acquisition system.

\subsubsection{Vision Acquisition and Automated Motion Tracking}

For untethered microrobot experiments, the laser beam was focused onto the ChevBot with sufficient power to initiates gating motion, which in turn causes the robot to escape from the laser beam waist. At that point the microrobot loses power, the laser beam needs to be repositioned to track the motion of the robot. Typical laser trackers will reposition the beam either through a series of movable mirrors, or through physical repositioning of the laser beam source. In a novel twist, we keep the laser beam position fixed, but we reposition the robot to the laser spot using a visual servoing scheme implemented by camera feedback and described in Figure 3-7.

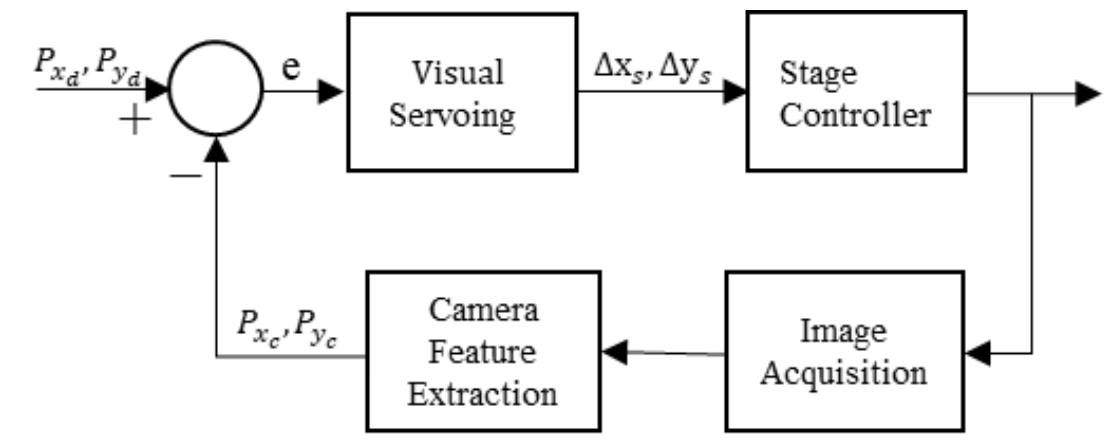

Figure 3-7 Control scheme of the combined laser-beam robot motion. 
Our vision feedback scheme is based on the NI smart camera which contains a color sensor with resolution of 640 by 480 and an Intel® processor. The smart camera processes images and extracts the pixel location of the microrobot with the pattern matching feature, then the pixel location is sent to a laptop PC that runs LabVIEW@ to drive the X-Y stages to maintain the microrobot under the laser spot.

An Image Jacobian was identified for visual servoing, which bridges the mapping between sampled pixel position to the X-Y stage's displacement. The Jacobian was estimated with seven randomly picked locations under the observation of the camera. If $\mathrm{P}_{\mathrm{x}}$ and $\mathrm{P}_{\mathrm{y}}$ are the position of the centroid of the microrobot feature observed under the camera, the image Jacobian has 4 entries that can be estimated, and connects the pixel variations to the stage position variation according to:

$$
\begin{gathered}
{\left[\begin{array}{l}
\Delta P_{X} \\
\Delta P_{Y}
\end{array}\right]=J\left[\begin{array}{l}
\Delta X \\
\Delta Y
\end{array}\right]} \\
J=\left[\begin{array}{ll}
J_{11} & J_{12} \\
J_{21} & J_{22}
\end{array}\right]
\end{gathered}
$$

in which $\mathrm{J}$ is the $2 \mathrm{x} 2$ image Jacobian, $\Delta \mathrm{P}$ is the difference of current position to previous position in pixels, and $\Delta \mathrm{X}$ and $\Delta \mathrm{Y}$ are variations in the $\mathrm{X}-\mathrm{Y}$ stage coordinates expressed in encoder counts.

In order to maintain the ChevBot at the center of the camera image, where the laser has been focused, the visual servoing feedback provides current pixel location of the ChevBot $\left(P_{x_{c}}, P_{y_{c}}\right)$, then compared with the desired pixel location $\left(P_{x_{d}}, P_{y_{d}}\right)$ to generate an error term used to drive the stages to a new location using a proportional controller: 


$$
\left[\begin{array}{c}
X_{n e w}-X_{c} \\
Y_{\text {new }}-Y_{c}
\end{array}\right]=\Delta s J^{-1}\left[\begin{array}{c}
P_{X_{d}}-P_{X_{c}} \\
P_{Y_{d}}-P_{Y_{c}}
\end{array}\right]
$$

where $\Delta \mathrm{s}$ is a parameter step-size of 0.5 and $J^{-1}=\left[\begin{array}{cc}-0.0089 & -0.0007 \\ -0.0011 & -0.0076\end{array}\right]$, as identified through calibration.

\subsection{Modeling of ChevBot}

This section presents the simulation results of the multi-physics modeling of ChevBot, which studies the opto-thermal-mechanical behavior under actuation of different levels of laser power and repetition rate. The results of this characterization are integrated in developing stick and slip model, which is also proposed and validated here. The stick-andslip model describes the 1-dimensional motion of an untethered ChevBot and estimates the velocity of ChevBot on a flat surface.

\subsubsection{Multi-Physics Modeling}

A multi-physics model was constructed and implemented using MATLAB Simulink® to simulate the opto-thermal-mechanical behavior of ChevBot. The model has three components: an optical heating model, a thermal dissipation model that describing heat distribution process in the robot's body with boundary conditions, and a mechanical expansion model that based on the resulting thermal loading [54]. The constants used in the simulation is shown in Table I. This model considered the case of the microrobots tethered to the substrate for comparison with actuator's displacement measurements. 


\section{Table I}

Values of the Constant Used in the Simulation of the Multi-Physics Analysis

\begin{tabular}{|c|l|c|}
\hline Constant & \multicolumn{1}{|c|}{ Quantity/Name } & Value \\
\hline$R$ & Reflectivity of Silicon & 0.3 \\
\hline$T_{e n v}$ & Environment Temperature & $20{ }^{\circ} \mathrm{C}$ \\
\hline$\rho_{S i}$ & Silicon Density & $2328 \mathrm{~kg} \cdot \mathrm{m}^{-3}$ \\
\hline$h_{\text {air }}$ & Air convection constant & $10 \mathrm{~W} \cdot\left(\mathrm{m}^{2} \cdot \mathrm{K}\right)^{-1}$ \\
\hline$k_{\text {air }}$ & Air thermal conductivity & $0.025 \mathrm{~W} \cdot(\mathrm{m} \cdot \mathrm{K})^{-1}$ \\
\hline$c_{v-\text { air }}$ & Air specific heat & $716 \mathrm{~J} \cdot(\mathrm{Kg} \cdot \mathrm{K})^{-1}$ \\
\hline$k_{S i}$ & Silicon thermal conductivity & $124 \mathrm{~W} \cdot(\mathrm{m} \cdot \mathrm{K})^{-1}$ \\
\hline$c_{v, S i}$ & Silicon specific heat & $702 \mathrm{~J} \cdot(\mathrm{kg} \cdot \mathrm{K})^{-1}$ \\
\hline$\alpha_{S i}$ & Silicon coefficient of thermal expansion & $2.6 \times 10^{-6}\left({ }^{\circ} \mathrm{C}\right)^{-1}$ \\
\hline$\theta$ & Theta (beam angle) & $0.05 \mathrm{rad}$ \\
\hline
\end{tabular}

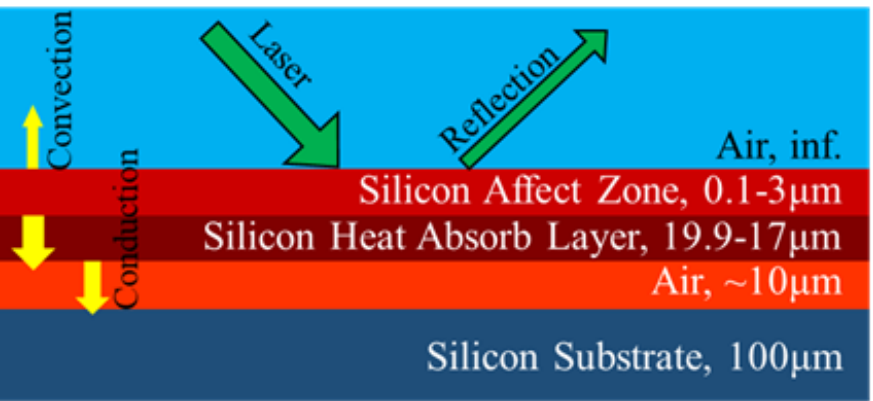

Figure 3-8 Block diagram of simulation model.

When laser is directed onto the thermal actuator, a portion of radiation energy is converted to heat actuator, while the rest is reflected and lost (Figure 3-8). The heat energy Q generated at the silicon surface can be described as [101] [102] [103]:

$$
d Q=(1-R) E_{e} \cdot A
$$

where the $\mathrm{R}$ is the surface reflectivity, $\mathrm{E}_{\mathrm{e}}$ is the irradiation in $\mathrm{J} / \mathrm{m}^{2}$ and $\mathrm{A}$ is the laser spot area in $\mathrm{m}^{2}$.

In Simulink®, this effect was simulated with the help of lumped first order model which assumes that the illuminated volume of the microrobot can be lumped into a hexahedron shaped silicon structure with the same thickness and surface area, thus the hexahedron 
becomes the equivalent of the laser heated part of the thermal actuator. The laser induces a temperature change in the silicon according to:

$$
\frac{d T}{d t}=\frac{d Q}{c \cdot m}, m=\rho V, V=\tau A
$$

where $\tau$ is the thickness of the material, $\mathrm{c}$ is the specific heat capacity and $\rho$ is the material's density. A portion of converted heat is lost due to thermal conduction, convection and radiation. The air gap between the microrobot actuator and the substrate is less than $10 \mu \mathrm{m}$, which causes heat loss by thermal conduction. The prevalence of conduction over convection has long been established in electrothermal MEMS actuation with a thin gap from the substrate [54] [101] [102]. The top surface of the microrobot has direct contact with air, resulting in heat losses due to convection; and losses due to radiation are assumed to be negligible. The thermal conduction and air convection are governed by:

$$
\text { Thermal Conduction: } \frac{d T}{d t}=-\frac{k}{\rho \tau^{2} c}\left(T-T_{\infty}\right)
$$

$$
\text { Air Convection: } \frac{d T}{d t}=-\frac{h}{\rho \tau c}\left(T-T_{\infty}\right)
$$

where the $\mathrm{k}$ is thermal conductivity, $\mathrm{T}_{\infty}$ is environment temperature, and $\mathrm{h}$ is the convection constant. After determination of the heat transfer extracted from above model, the displacement caused by thermal expansion can be evaluated. The total deformation of the shuttle can be expressed by [64]:

$$
\Delta L=\alpha \Delta T L
$$




$$
\Delta d_{T}=\left[L^{2}+2 L(\Delta L)-L \cos ^{2}(\theta)\right]^{\frac{1}{2}}-L \sin (\theta)
$$

where $\Delta \mathrm{L}$ is the length change on the beam due to temperature change, $\mathrm{L}$ is original length of the beam of the actuator, $\alpha$ is the thermal expansion coefficient, and $\theta$ is the acute angle of the beam formed with the shuttle on the thermal actuator. Finally, the expected force output of the actuator is given by [68] [104]:

$$
F_{a}=\frac{2 N A E \sin ^{2}(\theta)}{L} \Delta d_{T}
$$

where the $\mathrm{N}$ is number of beams of the actuator -6 pairs in our case. $\mathrm{A}$ is cross-section area; E is Young's modulus of Silicon.

\subsubsection{Stick and Slip Model}

The dynamical model of the ChevBot was approximated as a simple rigid body consisting of two sections attached at point $\mathrm{C}$, shown in Figure 3-9. The AC section represents the Chevron actuator with variable length $\mathrm{L}+\Delta \mathrm{d}$, and $\mathrm{BC}$ is the constant height (h) of dimple. In this diagram, the operating substrate is along the $\mathrm{x}$ axis $(\mathrm{AB})$, and the independent variables are $\mathrm{x} 1$, the dimple's $\mathrm{x}$ coordinate, and $\mathrm{x}$, the center of mass (CMS) of the ChevBot. The shuttle's leg position $\mathrm{x} 2$ is considered as a dependent variable.

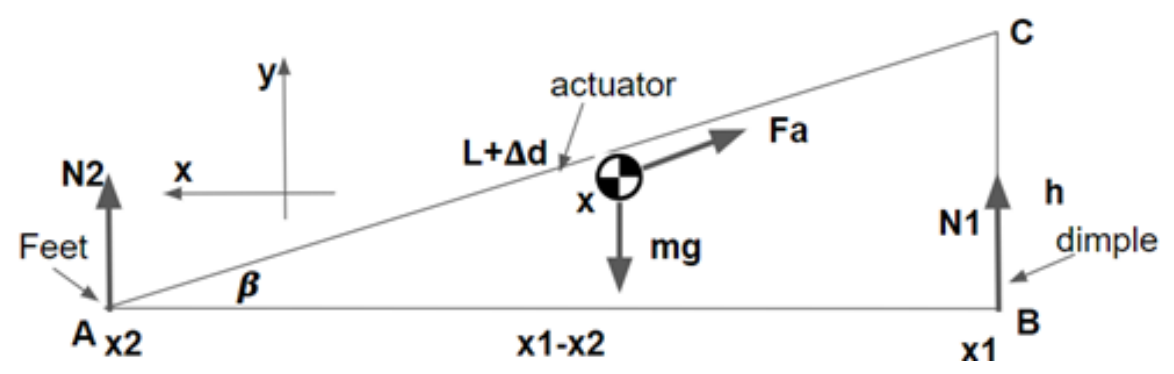

Figure 3-9 Free body diagram of ChevBot - sideview. 
The actuator deformation $\Delta \mathrm{d}$ from (6) generates instantaneous actuator force $\mathrm{F}_{\mathrm{a}}$ from (7) and can be used as input to the dynamic model. The tilt angle between microrobot and substrate $\beta$ is initially approximately $5^{\circ}$ and decreases during actuation and extension of the robot's body.

From the force and torque balance equation in the vertical y direction, the surface reaction forces $\mathrm{N}_{1}$ and $\mathrm{N}_{2}$ acting on the dimple and the leg can be determined. Assuming that the microrobot moves toward positive $\mathrm{x}$ direction, motion of the dimple $\left(\mathrm{x}_{1}\right)$ and overall body/CMS (x) can be described by:

$$
\begin{gathered}
F_{a} \cos (\beta)-\mu N_{1}-b \dot{x}_{1}=\frac{\left(x_{1}-x\right) m}{x_{1}-x_{2}} \ddot{x}_{1} \\
-F_{a} \cos (\beta)+\left(N_{1}-N_{2}\right) \mu-b \dot{x}=m \ddot{x}
\end{gathered}
$$

where $\mu=0.35$ is the coefficient of friction and $\mathrm{b}$ is the viscous damping $\mathrm{b}=6.8 \times 10^{-}$ ${ }^{5} \mathrm{~kg} / \mathrm{s} . \mu N_{1}$ and $\left(N_{1}-N_{2}\right) \mu$ represent frictional forces acting on the dimple and center of mass; $F_{a} \cos (\beta)$ is the $x$ component of the driving force, and $b \dot{x}_{1}, b \dot{x}$ are damping forces. Furthermore, the position of the microrobot's leg $x_{2}$ and angle $\beta$ (both dependent variables) can be determined from:

$$
x_{1}-x_{2}=\sqrt{(L-\Delta d)^{2}-h^{2}}, \sin (\beta)=\frac{h}{L-\Delta d}
$$

where $\mathrm{L}-\Delta \mathrm{d}$ is a function of time - the displacement of the thermal actuator due to laser irradiation.

The stick and slip motion can be simulated by solving (8) and (9) with respective constraint (10) - representing changes to the microrobot length $\left(\mathrm{x}_{1}-\mathrm{x}_{2}\right)$ and tilt angle $\beta$. 
Simulation is based on above equations of the motion with respective constraints were carried out in Simulink®. Figure 3-10 presents plots of the changes of the position of CMS, as a function of time for different values of the repetition frequency and the power density of the laser. The width of the laser pulse was kept constant (10ns in consistence with experimental parameters of our pulsed laser). Simulation results derived from our dynamic model (Figure 3-9) suggest that untethered ChevBot would move with a constant speed upon exposure to the laser light.

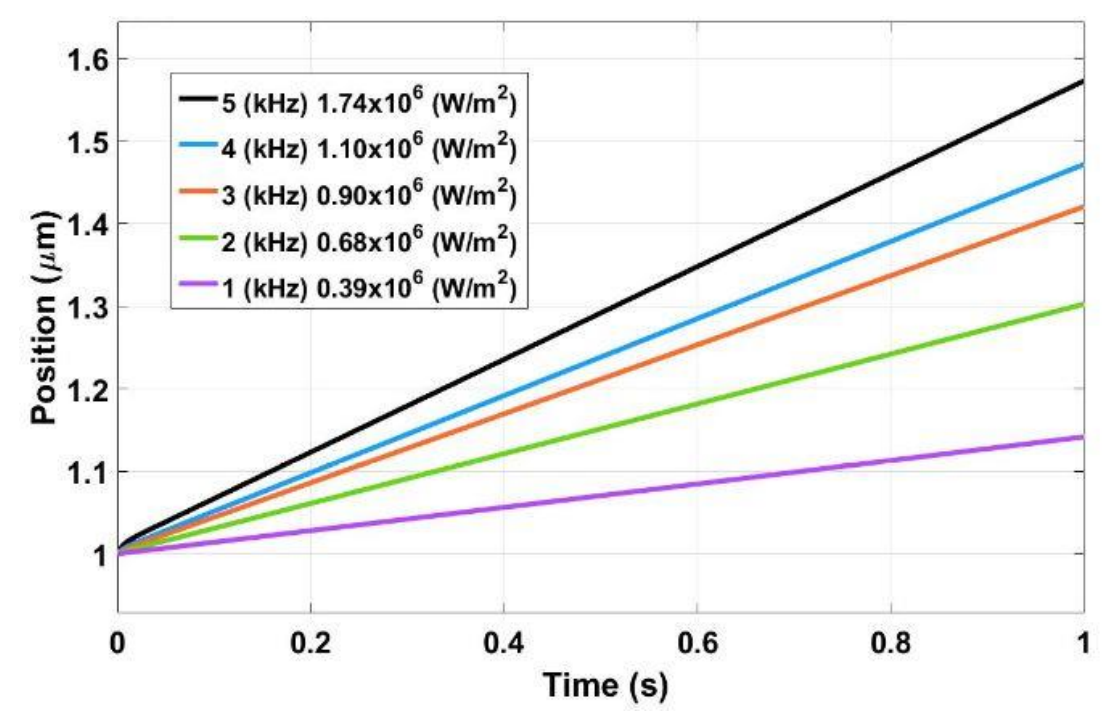

Figure 3-10 Changes of the position of center of mass ( $x$ ) as a function of time for different values of repetition frequency and irradiance.

From Figure 3-10, we notice that increasing the laser power density and repetition rate $f$ would increase the locomotion speed. Thus, indicating that by tuning the parameters of the laser (repetition rate and average power), it is possible to control dynamic properties of the ChevBot.

\subsection{Experimental Results and Discussion}

We conducted several experiments to confirm our ChevBot design choices and measure the microrobot resulting displacements and trajectories under laser power. 


\subsubsection{Experiments with tethered robots for model identification}

In this experiment, the robot actuator displacement was measured while the robot was tethered to the SOI device layer, which characterizes the actuator displacement and identifies components of a mass-spring-damper model. The repetition rate of the laser was set to $1700 \mathrm{~Hz}$ for maximum actuator displacement based on results from [69], and the laser diode current was considered as input to system identification. The system's output was the displacement of the thermal actuator, measured by the Keyence ${ }^{\circledR}$ displacement sensor.

A testing die was prepared such that many tethered ChevBots were located at the edge, and therefore the sensor laser beam can be reflected from the side wall of the thermal actuator to acquire the dynamic measurement. During this experiment, the laser module works under continuous mode and a pseudo random binary sequence (PRBS) was generated to toggle the laser diode, hence apply laser power to the microrobot, while the displacement sensor collected data at a sampling rate of $10 \mathrm{kHz}$. A LabVIEW® program was designed to collaborate the sensor and the laser module. Collected data is depicted in Figure 3-11 and reveals that thermal actuator displacements of 300nm are achievable with this level of power output, which also suggests that the minimum step size of the microrobot falls in this range. If the laser power is reduced, the achievable minimum microrobot step sizes can also decrease. 


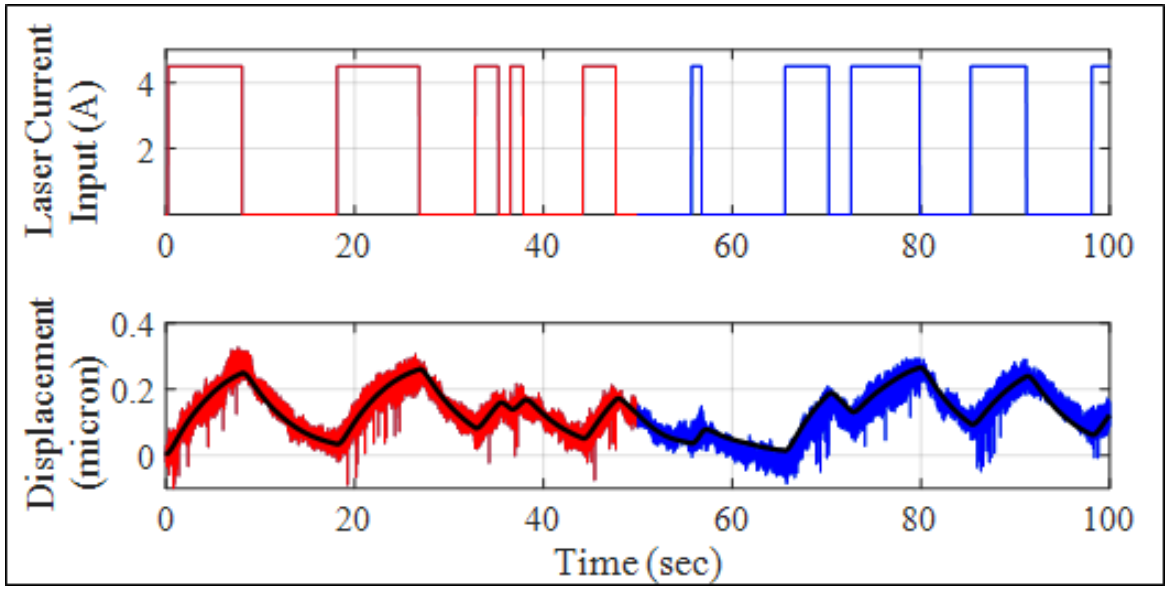

Figure 3-11 Laser power stimulus as input (top) and ChevBot thermal actuator displacement as output (bottom). The red and blue graphs represent the estimation and validation datasets, respectively. The black fitting line is the output of identified model to the PRBS input.

In the tethered configuration, the ChevBot's frame is stationary, and the actuator and dimple can be modeled as a mass-spring-damper system. Therefore, by taking the thermal aspect of the system's behavior into account, a third-order transfer function is expected to properly explain the overall dynamic behavior of the system from the laser actuation to the resulted displacement. Using MATLAB's system identification toolbox, a third-order transfer function was fitted to the estimation dataset. The fitness score of the estimation and validation datasets were 0.66 and 0.62 , respectively, and the resulting transfer function obtained was:

$$
H(s)=\frac{0.195}{(s+0.196)\left(s^{2}+5.096 s+13.95\right)}
$$

However, in order to check for model overfit, another system identification was performed fitting a first-order transfer function into the estimation dataset, resulting in a similar fit via: 


$$
H(s)=\frac{0.014}{(s+0.196)}
$$

The fitness score of the estimation and validation datasets were 0.65 and 0.60 , respectively. As a result, we concluded that our fitted first order model captures the thermal behavior of the chevron actuator, and that the mechanical modes of the system are not excited by this laser pulse frequency. Next, utilizing the stick-and-slip model introduced in [69], we simulated the untethered motion of the ChevBot by introducing a chevron actuator force obtained from laser power filtered through $\mathrm{H}(\mathrm{s})$. The microrobot mechanical system was represented by a double mass-spring-damper with values obtained from the microrobot geometry, in particular, the spring constant $\mathrm{K}=624 \mathrm{~N} / \mathrm{m}$ and masses $\mathrm{M}_{\mathrm{foot}}=1.1 \mu \mathrm{g}$, $\mathrm{M}_{\mathrm{body}}=3.3 \mu \mathrm{g}$. Although the surface condition represented by stiction and friction will vary, a dynamic model of the ChevBot using a Coulomb static and dynamic friction model with coefficients $\mu_{\mathrm{s}}=0.4$, and $\mu_{\mathrm{d}}=0.33$ predicts that locomotion velocities of $53 \mu \mathrm{m} / \mathrm{s}$ can be expected.

\subsubsection{Experiments with mobile ChevBots}

In the second series of experiments, assembled ChevBots were actuated on a silicon substrate by burst mode laser power. Each experiment took a few minutes, until the microrobot reaches the edge of the arena or its motion was blocked by dust particles. In general, experiments confirm that the ChevBot microrobot generates a straight trajectory heading towards its front direction, as shown in Figure 3-12 with all trajectories beginning from the origin. Five sets of experiments were conducted to determine the effect of different laser parameters and surface condition on speed (Table II). The trajectory plots of 
the ChevBot were recorded while the microrobot was tracked using both the $\mathrm{X}$ and $\mathrm{Y}$ encoder readings of the stages, and the servoing camera image coordinates.

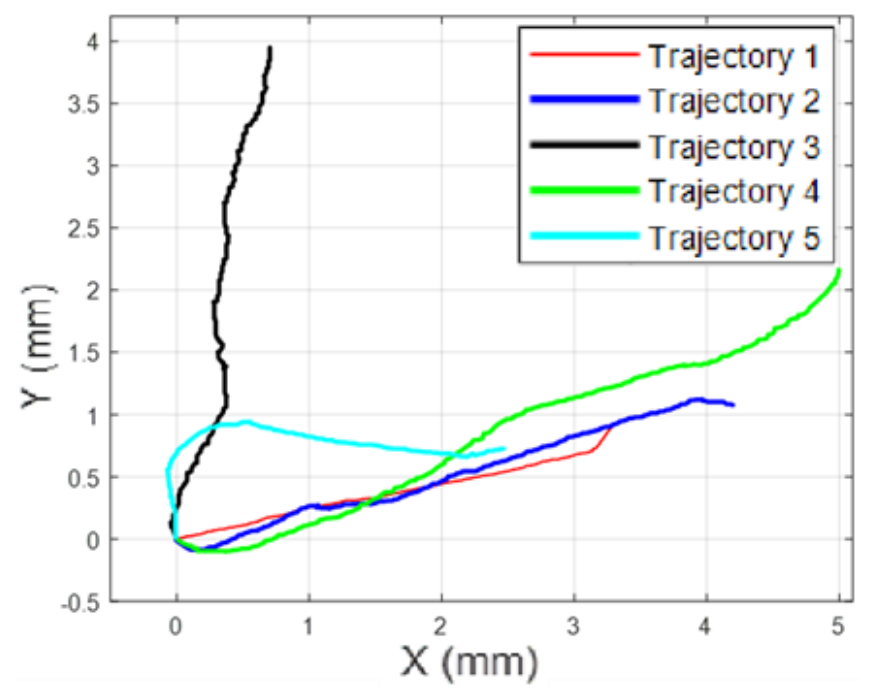

Figure 3-12 ChevBot's trajectory from 5 different experiments, mea sured within a time span of 90 seconds.

As most of the trajectories illustrate, ChevBot does not follow ideally straight path, experiencing sudden turns and stops. Such behavior can be caused by variations in surface conditions suggesting that the microrobot encounters dust specks or stiction patches on the substrate. It can also be caused by non-uniform irradiation of the Chevbot's body due to the elliptical shape of the laser beam's spot, as well as its position relative to the microrobot. Clarifying the importance of these effects needs more study in our future work.

As summarized in Table II, the robot velocity measured from the trajectory data, is reported as an average along with standard deviation $(\Sigma)$. The relatively large standard deviation suggests that measuring velocity using stage encoders is inaccurate. As a result, velocity was also measured from video recording of the experiment for a more accurate estimation, generally in the range of 20 to $110 \mu \mathrm{m} / \mathrm{s}$. The velocity of the microrobot and 
amount of energy it receives show a positive correlation, e.g. velocity increases when more power is delivered.

\section{Table II}

Untethered Locomotion Measurement Results

\begin{tabular}{|c|c|c|c|c|c|c|}
\hline Test & $\begin{array}{c}\text { Pulses } \\
\text { Per Burst }\end{array}$ & $\begin{array}{c}\text { Burst } \\
\text { Delay }\end{array}$ & $\begin{array}{c}\text { Avg. } \\
\text { Power }\end{array}$ & $\begin{array}{c}\text { Stage } \\
\text { Velocity }\end{array}$ & $\boldsymbol{\Sigma}$ & $\begin{array}{c}\text { Measured } \\
\text { Speed }\end{array}$ \\
\hline 1 & 30 & $200 \mathrm{~ms}$ & $388 \sim 454 \mathrm{~mW}$ & $39.1 \mu \mathrm{m} / \mathrm{s}$ & 26.1 & $21.8 \mu \mathrm{m} / \mathrm{s}$ \\
\hline 2 & 40 & $200 \mathrm{~ms}$ & $388 \sim 454 \mathrm{~mW}$ & $23.6 \mu \mathrm{m} / \mathrm{s}$ & 10.6 & $32.2 \mu \mathrm{m} / \mathrm{s}$ \\
\hline 3 & 40 & $100 \mathrm{~ms}$ & $410 \sim 468 \mathrm{~mW}$ & $51.2 \mu \mathrm{m} / \mathrm{s}$ & 36.7 & $90.6 \mu \mathrm{m} / \mathrm{s}$ \\
\hline 4 & 50 & $100 \mathrm{~ms}$ & $432 \sim 490 \mathrm{~mW}$ & $65.4 \mu \mathrm{m} / \mathrm{s}$ & 24.0 & $83.4 \mu \mathrm{m} / \mathrm{s}$ \\
\hline 5 & 50 & $50 \mathrm{~ms}$ & $440 \sim 504 \mathrm{~mW}$ & $90.3 \mu \mathrm{m} / \mathrm{s}$ & 40.4 & $109.0 \mu \mathrm{m} / \mathrm{s}$ \\
\hline
\end{tabular}




\section{ChAPTER 4 The SOlid ARTiculated Four AXEs Microrobot}

\subsection{Design of SAFAM Microrobot}

The microrobot was designed with two goals in mind, the functionality and assembly simplicity. sAFAM is inspired by its predecessor Articulated Four-Axes Microrobot (AFAM) and we used similar yet modified driving mechanism so that it kept the same functionality and reduced assembly complexity.

\subsubsection{Microrobot Components}

A fully assembled sAFAM microrobot has three major components: the two MEMS actuator banks, providing in-plane $\mathrm{x}-\mathrm{y}$ motion; the monolithic arm assembly, which is a single piece of silicon containing heterogenous features to convert in-plane actuator motion onto the end-effector; and a die carrier that interconnects power to the microrobot.

The arm assembly has five components: a cantilever arm, a serpentine spring, a thin beam spring, and the front and rear Zyvex stands. Figure 4-1(a) depicts the arm assembly design with typical dimensions in microns and it is fabricated on the same silicon wafer as the rest of the parts. The arm assembly has a $100 \mu \mathrm{m}$ tall, $2.954 \mathrm{~mm}$ long cantilever beam with a round tip, which allows for future mounting of tools such as an AFM probe. The fixed end of the cantilever beam is connected with two springs: the serpentine-shaped spring containing 8 turns with $20 \mu \mathrm{m}$ radius allows deflection on both lateral (X and $\mathrm{Y}$ axes) and axial ( $\mathrm{Z}$ axis) directions, and connects the arm with the front Zyvex stand; a thin-beam spring with dimensions of $10 \mu \mathrm{m}$ width and $2487 \mu \mathrm{m}$ length, provides an axial stiffness 
greater than its lateral stiffness to transmit pushing and pulling forces from the actuator banks to the end-effector.

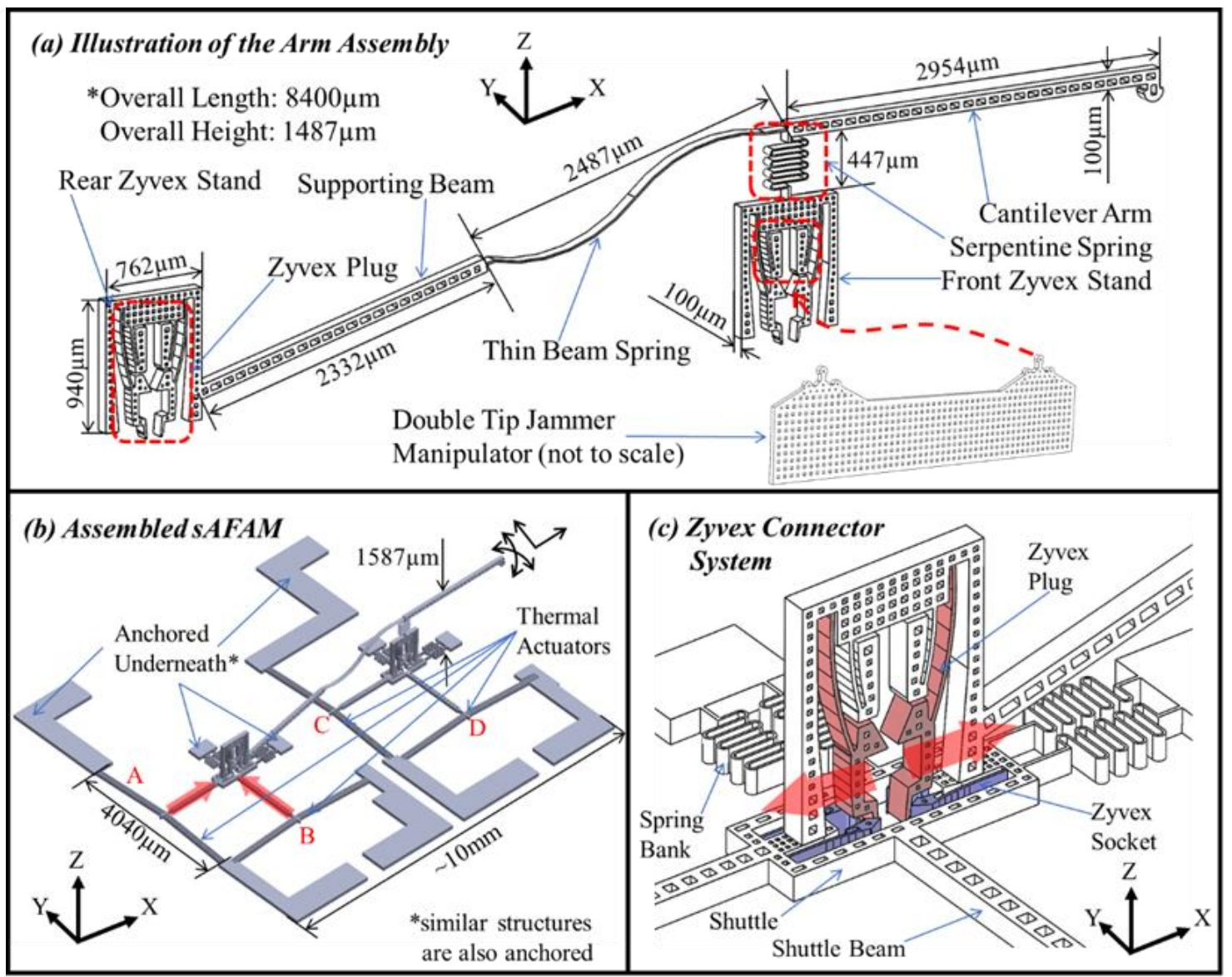

Figure 4-1 Design illustration of SAFAM. (a) Side view of the arm assembly with dimension and naming of each critical part. (b) Illustration of a fully assembled sAFAM microrobot, with name of the actuators marked red. (c) Zoom-in view of the rear Zyvex connector.

The Zyvex connector system provides a means to assemble the arm piece to the MEMS $\mathrm{X}-\mathrm{Y}$ stages and further transmit motion from the actuator to the end-effector, as shown in Figure 4-1 (b). The connector system's major two components are shown in Figure 4-1(c): the plug and the socket. During assembly, a jammer opens the claws marked by red along the direction indicated by arrow, and the blue claws of the socket opens then interlock to each other [71] [72]. The detailed design and analysis of these connectors have been studied 
extensively in the past and shown to be effective out-of-plane couplers for micro mechanisms and micromirrors [43] [41] [71] [72] [29].

To generate motion, the sAFAM uses two in-plane actuator banks consisting of coupled X-Y micro mechanisms shown in Figure 4-1 (b). The two in-plane X-Y stages are anchored on the substrate by a $2 \mu \mathrm{m}$ thick buried-oxide (BOX) layer under the electrodes. On each X-Y stage, three anchors are coated with gold contacts and two pairs of thermal actuators are fabricated between them. The width of the thin beams of the thermal actuator is $10 \mu \mathrm{m}$ and they form an $86.57^{\circ}$ angle with the anchor. A shuttle with a Zyvex socket is located at the center of the X-Y stage, which connects to the thermal actuators through a shuttle beam. On the other side of the shuttle, two pairs of anchored serpentine springs support the shuttle by lifting it and preventing it from touching the substrate. The two X-Y stages are identical in shape and orientation, and their shuttles are aligned so the arm assembly can be mounted onto them. Since the thermal actuators can only be actuated toward their bending direction, they must be arranged in the same orientation to achieve 4 DOF. The choice of these geometric parameters is discussed in more detail in [29].

Each X-Y stage has two thermal actuators connected to the center shuttle. When one actuator is engaged, the shuttle moves along the actuation direction, shown as the red arrows in Figure 4-1(b), while dragged by the other pair of the actuator. Then this motion is coupled to the arm assembly through the Zyvex stands. By engaging different actuators, a different motion pattern on the arm assembly is achieved. To clarify discussion, each actuator is named as A, B, C and D, as indicated in Figure 4-1 (b).

sAFAM has 4 DOFs, as it enables tip motion in yaw, pitch, translational $\mathrm{X}$ and $\mathrm{Y}$ directions, as described in the coordinate system (XYZ reference frame) defined in Figure 
4-1 (b). The pitch motion with an axis of rotation aligned with the Y direction generates coupled tip displacement in the $\mathrm{X}$ and $\mathrm{Z}$ direction. The yaw motion has its axis of rotation aligned with the $\mathrm{Z}$ direction generates coupled tip displacement in the $\mathrm{X}$ and $\mathrm{Y}$ direction. Pitch and yaw motion can be realized thanks to the serpentine spring supporting cantilever arm depicted in Figure 4-1 (a), which acts as a 2-dimensional rotation joint. The thin beam spring can be modeled as a cantilever that does not compress or elongate easily but is able to bend laterally by external force. The pitch motion is generated by engaging either actuator $\mathrm{A}$ or $\mathrm{C}$. When $\mathrm{A}$ is engaged, the distance between the two Zyvex stands becomes smaller, and the thin beam spring gives the cantilever arm a forward movement and the end-effector pitches towards substrate, e.g. in the $-\mathrm{Z}$ direction. When $\mathrm{C}$ is engaged, it similarly generates a pitch motion while the end-effector tilts upward in the $+Z$ direction. When actuators B or D are powered, the serpentine spring serves as a pivot, allowing the thin-beam to drive the end-effector towards $-\mathrm{Y}$ or $+\mathrm{Y}$ direction in $\mathrm{XY}$ plane as the endeffector yaws. Finally, to generate a translational forward motion in the $+\mathrm{X}$ direction, actuators A and C are engaged simultaneously. Similarly, when actuator B and D are engaged at the same time, the end-effector moves towards $+\mathrm{Y}$ direction to generate lateral translational motion. A detailed kinematic model for AFAM, confirming its 4-DOF positioning capability was studied by Z. Yang et al. in [105].

\subsubsection{Assembly Methodology}

The construction process of sAFAM undergoes two major steps: preliminary part assembly and permanent attachment using ultra-violet curable epoxy adhesive. To successfully assemble the arm assembly into the two in-plane X-Y stages on the substrate, a double tip jammer microgripper is used as depicted in Figure 4-1 (a). It includes two 
round tips of $75 \mu \mathrm{m}$ diameter and a rectangle body. The distance between the two jammer tips is $5.050 \mathrm{~mm}$, which is the same as the distance between the two Zyvex stands on the arm assembly and the distance between the two Zyvex sockets on the X-Y stage banks.

With the help of the jammer manipulator, the arm assembly is picked up, reoriented and aligned with the assembly site (the Zyvex socket) on the substrate using controlled robotic positioning. Once aligned to desired socket location, the jammer performs a precise pushdown motion and snaps the arm into the substrate: the red dashed arrow in Figure 4-1 (a) shows the right jammer tip pairs with the front Zyvex plug. During part pick-up, the tip inserts into the upper half of the Zyvex plug; after alignment, the jammer pushes down and opens the lower part of the plug and interlocks the Zyvex connector system.

\subsection{Fabrication and Assembly}

The fabrication and assembly process of sAFAM, and its associated robotic hardware is described in this Section.

\subsubsection{Fabrication}

The sAFAM was fabricated on a Silicon on Insulator (SOI) wafer by MEMS process at the Micro/Nano Technology Center at University of Louisville. The SOI wafer has the following specification: $100 \mu \mathrm{m}$ device layer, $\mathrm{N}$ type $\langle 100\rangle, 0.01-0.02 \mathrm{ohm}-\mathrm{cm}$ resistivity, $2 \mu \mathrm{m}$ BOX and $500 \mu \mathrm{m}$ handle layer. Four major fabrication steps are involved: 1 . Metal deposition; 2. Deep reactive ion etching (DRIE); 3. MEMS part releasing with hydrofluoric (HF) acid followed by critical point drying (CPD); and 4. Preliminary probing before assembly.

Metal deposition process deposits a thick layer of gold (above 300nm) by PVD-75 thin film deposition system (Kurt J. Lesker Company, PA, USA) for 4 minutes under 300W 
DC power, which provides electrodes for final wire bonding to a package. To boost $\mathrm{Au}-$ Si bounding, an intermediate layer of chrome was also deposited for 2 minutes before depositing gold. Metal patterning was done by subtractive process: a light-field mask imposes the desired pattern onto positive photoresist, then baked and etched by gold and chromium etch under designated chemical benches. Another layer of photoresist SPR2203.0 (MicroChem Corp., MA, USA) was deposited and patterned with a dark-field mask featuring DRIE patterns and fully covers the metal that been deposited. The photoresist used in this step must have enough thickness to withstand DRIE process to ensure the integrity of fine micromachined silicon features. And an extended baking procedure was preferred to drive the solvent out of photoresist, hence strengthening it.

DRIE (SPTS Technologies Ltd., Newport, UK) was performed in a timed and inspected manner: the etching rate was characterized by measuring 15 minutes etching depth, and the finial etching time was based on that number. Inspection on fine features such as releasing holes was needed to guarantee a successful micromachining.

Releasing with $49 \%$ hydrofluoric acid was performed after dicing the wafer by immersing each die into the acid. The releasing time for each die depends on their maximum undercut sizes, which ranges from 20 to 30 minutes. After release, samples are preserved in deionized (DI) water and immediately transferred to a critical point dryer (CPD) to remove liquid to prevent adhesion. In an improved recipe, vapor HF etching tool was used.

Detailed cleanroom fabrication recipe is attached in the appendix. 


\subsubsection{Assembly}

Assembly of the sAFAM was performed with NEXUS [106] microassembly station, shown in Figure 4-2. This system consists of two cameras with high, adjustable $(2 \mathrm{x}-10 \mathrm{x})$ power optics, one mounted at the top (EO-1312C, Edmund Optics Inc., Barrington NJ, USA) provides a top-down view; the other was mounted at side (EO-6412C) perpendicular to the top, providing a horizontal view. The cameras can track the jammer end-effector motion during rotations, de-tethering and assembly operations. The sample chuck was mounted on the base manipulator M1, and it can hold five dies at the same time and has vacuum channels to securely fix each die. The two computer-controlled micromanipulators, M1 and M2, provide a total of 9 degrees of freedom. M1 (consists of: ILS250CC, 443 Series and URS75BPP, all from Newport Corporation Irvine, CA, USA) provides motorized X-Y translation and rotation to the sample chuck; M2 (VP-25XL-XYZR and PR50CC, both from Newport Corporation Irvine, CA, USA) consists of a motorized X-Y$\mathrm{Z}$ translation stage and a rotation stage, and a mini manual $\mathrm{X}-\mathrm{Y}$ stage mounted on the rotation stage to adapt the end-effector, totally providing 6 degrees of freedom. An exchangeable end-effector was connected to the manual stage by magnetic connection. Illumination was provided with two fiber lamps with adjustable angle and brightness. 


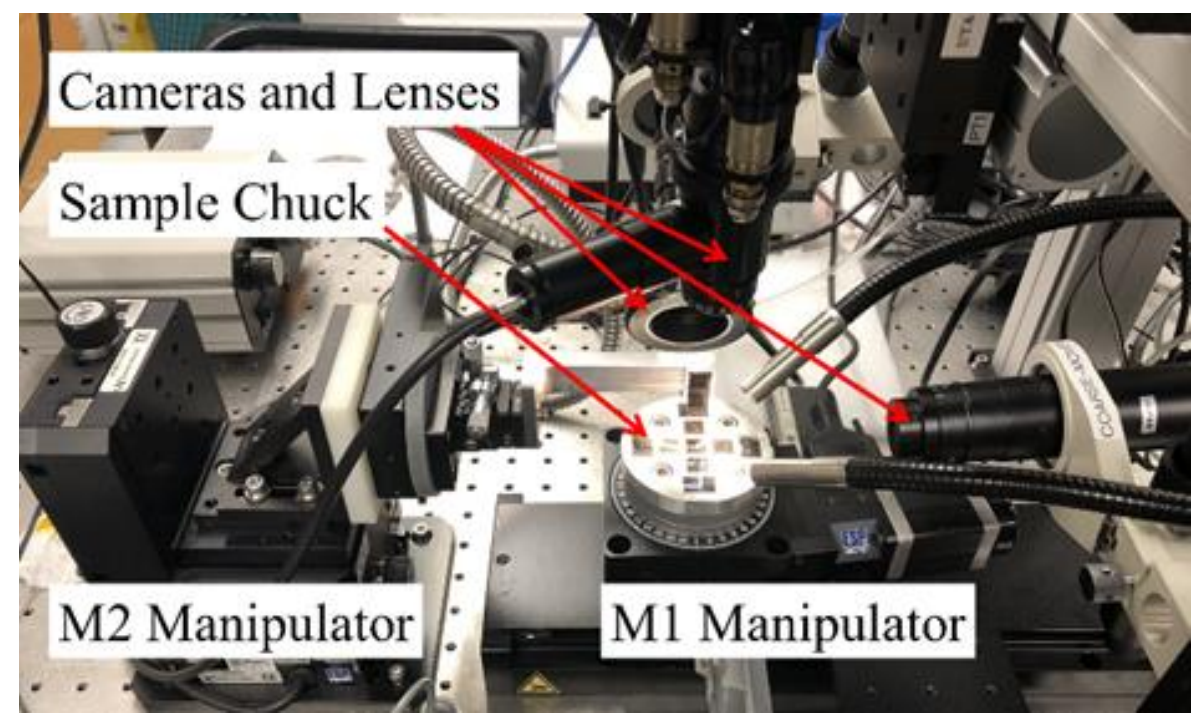

Figure 4-2 NEXUS microassembly station: $M 1$ and M2 manipulators; top camera: EO-1312C, side camera: EO-6412C.

The assembly process starts by fixing the double tip jammer onto the M2's end-effector of the microassembly station using Super 77 adhesive (3M, MN, USA). Next, we orient the jammer at a 90-degree angle to the substrate, allowing the tips to face downward. With the help of top camera, the operator roughly translates the jammer to the arm assembly, then fine tunes the orientation of the jammer to align with the tethers that hold the arm assembly in place. After breaking the MEMS tethers, the arm assembly was free to be picked up by the jammer through the snap-fastener, which is compliant and allows for alignment error mitigation in excess of $5 \mu \mathrm{m}$ [71] [72]. After the arm pick-up, the endeffector rotates 90 degrees again and align the arm assembly with the Zyvex socket, then insert the arm assembly into the Zyvex sockets on the MEMS X-Y stages.

Although the snap-fasteners are good temporary fixtures, they will not be able to withstand significant forces and torques without disassembly. As a result, the arm assembly was fixed onto the actuator banks using UV curable epoxy (Bondic $®$, Aurora, ON L4G 3V5, CA). A small amount of UV adhesive was brushed with a single optical fiber at 
critical locations of the snap fasteners after pre-assembly. A UV flashlight was then used to cure the epoxy immediately after dispensing. Figure 4-3 depicts a fully assembled and packaged SAFAM arm, which is now ready for the actuation cycling.

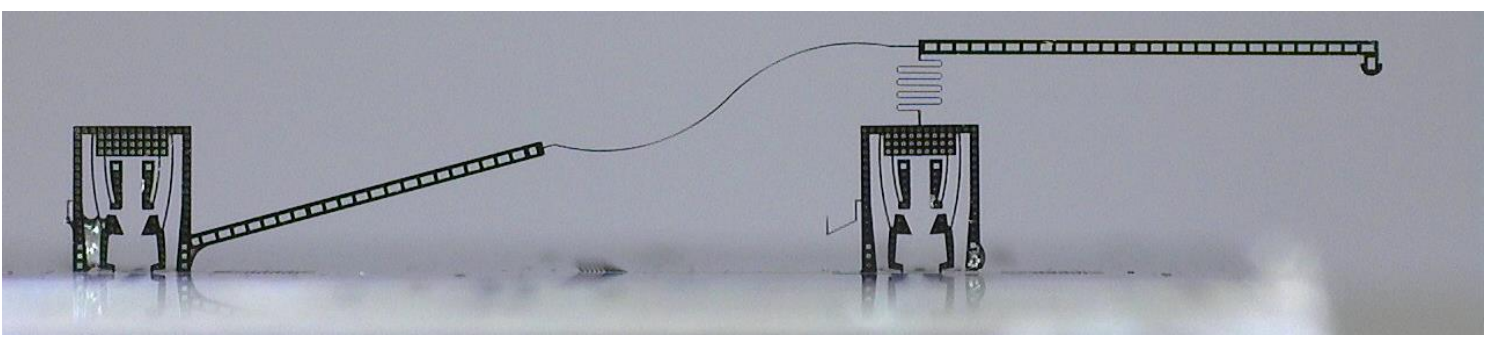

Figure 4-3 Side view of the assembled SAFAM arm.

\subsection{Finite Element Analysis of Microrobot}

In order to analyze and verify the functionality of sAFAM, a finite element analysis (FEA) was carried out using ANSYS®. The sAFAM photolithography mask was designed in Tanner L-EDIT®, then exported into a .dxf file. 3D models of microrobot components were then extruded in SolidWorks® by introducing corresponding .dxf files. Finally, a 3D solid model in .sldasm format was imported into ANSYS® for FEA simulation.

\subsubsection{The Experiment Assisted Simulation Method}

The performance of the MEMS X-Y stage was previously studied in detail in numerous articles [106] [104] [107] [108], which describe both its displacement and force output. The geometry of the thermal-actuator banks of sAFAM is shown in Figure 4-1 (b). The five Chevron beams have a length of $2004 \mu \mathrm{m}$, width of $12 \mu \mathrm{m}$, and thickness of $100 \mu \mathrm{m}$. They are spaced $18 \mu \mathrm{m}$ apart. The length of the shuttle beam is $1790 \mu \mathrm{m}$. As reported in [29], this actuator bank is capable of generating $50 \mathrm{mN}$ of force and $48 \mu \mathrm{m}$ of displacement if not coupled with in-plane serpentine springs. Furthermore, the $\mathrm{X}$ and $\mathrm{Y}$ axes are stiffened 
by adding in-plane spring banks with a torsional stiffness of $0.12 \mathrm{Nm} / \mathrm{rad}$ and $0.26 \mathrm{Nm} / \mathrm{rad}$ for in-plane and out-of-plane motions, respectively.

In general, electrothermal actuator simulation requires accurate information about the boundary conditions, such as substrate temperature, environmental air temperature, actuation voltage etc. In addition, a coupled thermal and electrical analysis needs to be undertaken. In this work, we conducted experimental testing to obtain ranges for X-Y stage actuation displacement to avoid complex coupled simulations. Specifically, after fabrication and packaging, a range of pulse width modulated voltages from a $25 \mathrm{~V}$ power supply was applied onto the electrodes of the thermal actuators, while displacement measurements of the Zyvex socket were recorded with a camera and microscopic lens set. Resulting thermal actuator displacement in both $\mathrm{X}$ and $\mathrm{Y}$ directions varied from 0 to $20 \mu \mathrm{m}$. However, for some actuators when the input voltage passes $15 \mathrm{~V}$, the actuator beams start to suffer buckling effects and the displacement of the center shuttle drops, which ANSYS simulation cannot cover. Therefore, the displacement inputs used in the simulation for each actuator was below the buckling threshold. A comparison of the resulting simulation and experimental results obtained are discussed in detail in Chapter 4.3.4.

\subsubsection{Stiffness Analysis of the Thin Beam Spring and the Serpentine Spring}

As stated in Chapter 4.1.1, the two springs translate the in-plane motion onto the pitch and yaw motion of the cantilever arm. This phenomenon is confirmed with the help of ANSYS simulation. To observe the pitch motion, actuator A was activated with $10 \mu \mathrm{m}$ of displacement as simulation input, while the yaw motion was observed with $10 \mu \mathrm{m}$ displacement input on actuator B. The simulation result is shown in Figure 4-4, which 
depicts the deformation on the joint of the two springs: the wireframe indicates the undeformed shape and the solid colored indicates the deformed shape.

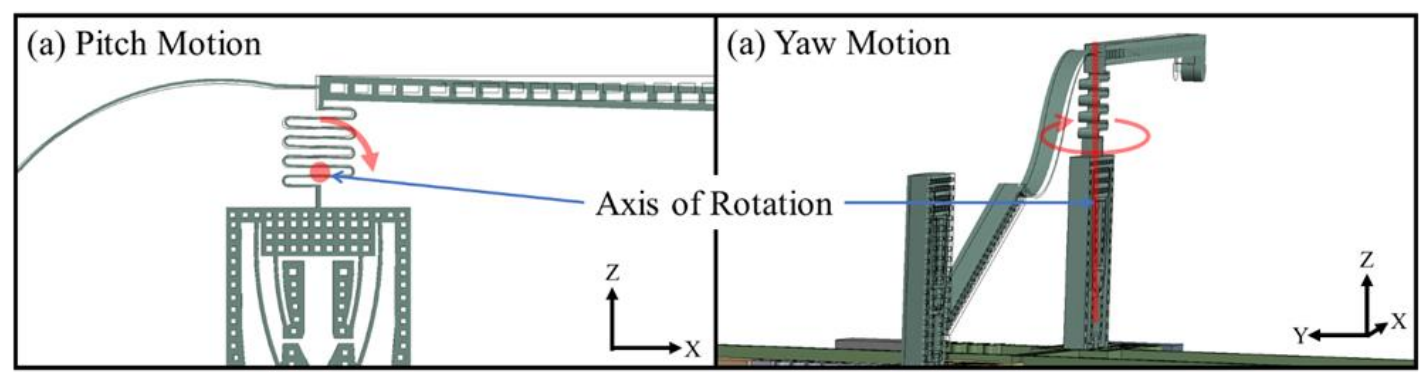

Figure 4-4 Original position (wireframe) vs. actuated position (solid colored). (a) When actuator $A$ is engaged with $10 \mu \mathrm{m}$ displacement, the axis of rotation is along $Y$ axis. The cantilever arm pitches down. (b) When actuator $B$ is engaged with $10 \mu \mathrm{m}$ displacement, the axis of rotation is along $Z$ axis. The cantilever arm yaws. The scale factor is set to 2 for demonstration purpose.

The magnitude of pitch and yaw depends on the design parameters of the two springs. Design parameters include the shape of the springs represented by the length of the springs, the radii of turns, and the separation distance between Zyvex connectors. To understand the effect of various geometric parameters of these springs, we carried out FEA simulations by assigning the "Fixed Support" boundary conditions and applying external forces to indicated regions shown in Figure 4-5. With this simulation data, we calculated stiffness coefficients along $\mathrm{X}, \mathrm{Y}$ and $\mathrm{Z}$ directions, and selected geometric parameters such that maximize the pitch and yaw range of motion. 


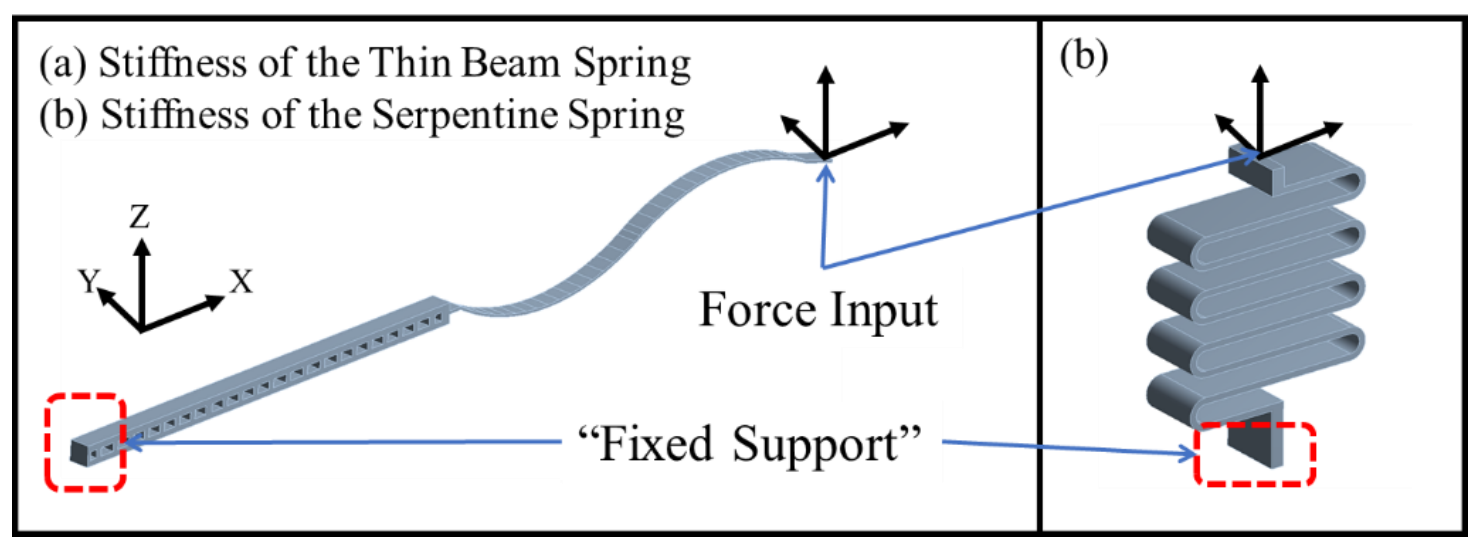

Figure 4-5 ANSYS simulation setup to study the stiffness of the thin beam spring and the serpentine spring.

The results shown in Table III correspond to the geometric parameters of the design shown in Figure 4-1. The axial stiffness of the thin beam in the $X$ direction was $76.68 \mathrm{~N} / \mathrm{m}$, which was significantly larger than the stiffness of the serpentine spring in the $\mathrm{X}$ direction $(10.04 \mathrm{~N} / \mathrm{m})$. This value was comparable to the stiffness of the serpentine spring in the $\mathrm{Z}$ direction $(66.34 \mathrm{~N} / \mathrm{m})$, creating a large pitch motion for the cantilever arm shown in Figure 4-4 (a). On the other hand, the yaw motion of the cantilever is due to the Y direction stiffness of the serpentine spring $(26.03 \mathrm{~N} / \mathrm{m})$ which is larger than $\mathrm{Y}$ direction stiffness of the thin beam spring $(5.56 \mathrm{~N} / \mathrm{m})$, so that when actuator B engages, the serpentine spring pivots rather than bent towards $\mathrm{Y}$ direction. When designing this arm mechanism, there will be trade-offs between the range of motion and the force output of the end-effector. In our design we emphasized maximizing the range of motion, rather than the amount of endeffector force. Although the in-plane thermal actuators can generate $\mathrm{mN}$ level forces, due to the low stiffness of the thin beam spring, sAFAM's end-effector transmits $\mu \mathrm{N}$ level forces to its end-effector. The force output of this design at maximum actuator deflections was $55 \mu \mathrm{N}$ in the pitch direction, $120 \mu \mathrm{N}$ in the yaw direction and $1 \mathrm{mN}$ in $\mathrm{X}$ translational direction. 


\section{Table III}

Simulation result of stiffness for the thin beam spring and the serpentine spring

\begin{tabular}{|c|c|c|c|c|c|c|}
\hline & \multicolumn{3}{|c|}{ Thin Beam Spring } & \multicolumn{3}{c|}{ Serpentine Spring } \\
\hline Deformation \& Force Direction & $\mathrm{X}$ & $\mathrm{Y}$ & $\mathrm{Z}$ & $\mathrm{X}$ & $\mathrm{Y}$ & $\mathrm{Z}$ \\
\hline Force Input $(\mu \mathrm{N})$ & 10 & 10 & 10 & 50 & 50 & 50 \\
\hline Deformation & 0.1304 & 1.7972 & 21.2110 & 4.9792 & 1.9208 & 0.7537 \\
\hline Stiffness $(\mathrm{N} / \mathrm{m})$ & 76.68 & 5.56 & 0.47 & 10.04 & 26.03 & 66.34 \\
\hline
\end{tabular}

\subsubsection{Stress Analysis of the Arm Assembly}

Stress distribution is an essential factor in the reliability of the sAFAM. Silicon can withstand 7GPa of stress measured by Von Mises Method [109]. With maximum actuator displacement as boundary condition added to the structure, the maximum stress point of the whole structure was found at the supporting springs on the in-plane X-Y stage of $139 \mathrm{MPa}$, while the highest stress reading of 49MPa was found on the arm assembly of the thin beam spring when actuator B was engaged. Both readings are much smaller than 7GPa. These results suggest that the considered geometry of the sAFAM's structure might be able to withstand a significant number of actuation cycles, while the exact number of actuations needs to be confirmed experimentally.

\subsubsection{Simulation of Microrobot Tip Displacement}

The goal of this simulation is to determine the relationship between actuator displacement and sAFAM's tip motion. Red arrows in Figure 4-1 (b) denote the direction of displacement input on the thermal actuators in the simulation. In this model, as in the case of the assembled structure (Figure 4-1 (b)), the displacement inputs on each actuator are denoted as A, B, C and D.

In order to optimize the structural simulation, the multi-zone meshing method was used to generate an even mesh across the whole robot (76388 elements, above $91.5 \%$ elements 
have skewness below 0.5) with refined element size of $20 \mu \mathrm{m}$ at critical locations such as the thermal actuator beams, the serpentine spring and the thin beam spring. Although the silicon lattice orientation affects Young's modulus [110], an average value of $160 \mathrm{~Pa}$ was chosen in this simulation [111].

To maximize accuracy, the simulation was assisted with the results form displacement experiments with an assembled SAFAM structure. Two actuation voltages that under buckling threshold were selected to power each thermal actuator, with corresponding shuttle displacement been recorded by the top camera. These displacement data were then inserted into ANSYS model to evaluate the end-effector's displacement. For example, the pitch motion simulation was obtained by providing $\mathrm{X}$ direction input of $10 \mathrm{~V}$ on either actuator $\mathrm{A}$ or $\mathrm{C}$, then measuring displacement along the $\mathrm{Z}$ direction as $-43.6 \mu \mathrm{m}$ and $44.4 \mu \mathrm{m}$ respectively. The smaller displacement along $\mathrm{X}$ and $\mathrm{Y}$ directions is due to the motion coupling effect of the structure. Furthermore, yaw motion is generated by either engaging actuator $\mathrm{B}$ or $\mathrm{D}$ along the $\mathrm{Y}$ direction. Forward motion $(+\mathrm{X})$ is generated by engaging $\mathrm{A}$ and $\mathrm{C}$ together and lateral (-Y) motion is generated by engaging B and D simultaneously.

Table IV rows 1 through 4 show the resulting simulated end-effector displacement along $\mathrm{X}, \mathrm{Y}$ and $\mathrm{Z}$ corresponding to those combinations of actuator inputs that create displacement primarily along those directions. The simulation shows that the end-effector is expected to translate approximately $13 \mu \mathrm{m}$ on $\mathrm{X}$-axis, yaws $47 \mu \mathrm{m}$ on $\mathrm{Y}$-axis and pitches $115 \mu \mathrm{m}$ on $\mathrm{Z}$ axis. 


\section{Table IV}

ANSYS simulation result depicting sAFAM tip displacements in $\mathrm{X}, \mathrm{Y}$ and $\mathrm{Z}$ direction as a function of actuator input voltages (under $\operatorname{row} \mathrm{X}, \mathrm{Y}$ and $\mathrm{Z}$ ), and corresponding experimental measurement results using optical microscopy $(0.535 \mu \mathrm{m} /$ pixel for $\mathrm{X}$ and $\mathrm{Y}$ direction, $0.503 \mu \mathrm{m} /$ pixel on $\mathrm{Z}$ direction. Under row $\mathrm{X}^{\prime}, \mathrm{Y}^{\prime}$ and $\mathrm{Z}^{\prime}$ )

\begin{tabular}{|c|c|c|c|c|c|c|c|c|c|c|c|c|}
\hline Axis & $\begin{array}{c}\mathbf{A} \\
(\mathbf{1 0 V})\end{array}$ & $\begin{array}{c}\mathbf{A} \\
(\mathbf{1 5 V})\end{array}$ & $\begin{array}{c}\mathbf{B} \\
(\mathbf{1 0 V})\end{array}$ & $\begin{array}{c}\mathbf{B} \\
(\mathbf{1 5 V})\end{array}$ & $\begin{array}{c}\mathbf{C} \\
(\mathbf{1 0 V})\end{array}$ & $\begin{array}{c}\mathbf{C} \\
(\mathbf{1 5 V})\end{array}$ & $\begin{array}{c}\mathbf{D} \\
(\mathbf{1 0 V})\end{array}$ & $\begin{array}{c}\mathbf{D} \\
(\mathbf{1 5 V})\end{array}$ & $\begin{array}{c}\mathbf{A C} \\
(\mathbf{1 0 V})\end{array}$ & $\begin{array}{c}\mathbf{A C} \\
(\mathbf{1 5 V})\end{array}$ & $\begin{array}{c}\text { BD } \\
(\mathbf{1 0 V})\end{array}$ & $\begin{array}{c}\text { BD } \\
(\mathbf{1 5 V})\end{array}$ \\
\hline $\begin{array}{c}\text { Input } \\
\text { Displ. } \\
(\mu \mathrm{m})\end{array}$ & 9.7 & 13.4 & 9.7 & 11.8 & 9.6 & 11.8 & 9 & 9 & $9.7,9.6$ & 13.4, & $9.7,9$ & $11.8,9$ \\
\hline $\begin{array}{c}\mathrm{X} \\
(\mu \mathrm{m})\end{array}$ & 7.0 & 9.7 & 0.5 & 0.6 & 2.9 & 3.5 & -0.3 & -0.3 & 9.6 & 12.9 & -0.3 & -0.2 \\
\hline $\begin{array}{c}\mathrm{Y} \\
(\mu \mathrm{m})\end{array}$ & 3.0 & 4.0 & -21.6 & -26.3 & -5.3 & -6.5 & 20.7 & 20.7 & -2.3 & -2.3 & -1.1 & -5.8 \\
\hline $\begin{array}{c}\mathrm{Z} \\
(\mu \mathrm{m})\end{array}$ & -43.6 & -60.2 & 0.3 & 0.3 & 44.4 & 54.7 & -8.2 & -8.2 & 0.1 & -7.2 & -8.6 & -8.6 \\
\hline $\begin{array}{c}\mathrm{X} \\
(\mu \mathrm{m})\end{array}$ & 7 & 12 & 0 & 0 & 2 & 2 & 0 & 0 & 9 & 14.5 & 0 & 0 \\
\hline $\begin{array}{c}\mathrm{Y}^{\prime} \\
(\mu \mathrm{m})\end{array}$ & 0 & 0 & -6 & -6 & -6 & -6 & 14 & 14 & 0 & 0 & 10 & 11 \\
\hline $\begin{array}{c}\mathrm{Z} \\
(\mu \mathrm{m})\end{array}$ & -12 & -26 & 0 & 0 & 13 & 23 & 0 & 0 & 6 & -6 & 0 & 0 \\
\hline
\end{tabular}

\subsection{Experimental Results: Microrobot Workspace}

\subsubsection{FEA Model Validation using Optical Microscopy}

The FEA simulation was conducted with thermal actuator displacement input determined from the experiments with a fully assembled sAFAM. To carry out these experiments, a custom made electronic PWM MEMS driver with LabVIEW® interface was developed to apply controlled voltages on the four actuator banks of the microrobot. A motor controller L298N (STMicroelectronics, Geneva, Switzerland) module was used to drive the thermal actuators by PWM signals generated from the interface. The power module was a voltage boost converter features XL6009 (XLSEMI Inc., Shanghai, China) chip, that can boost 12 VDC to 25VDC. A camera (EO-1312C) with zoomable lens was placed on top of the sAFAM to observe both the thermal actuator and the end-effector displacement on $\mathrm{X}$ and $\mathrm{Y}$ direction; the side camera (EO-6412C) was placed perpendicular 
to the top camera, which focused on the end-effector to observe the pitch motion or $\mathrm{Z}$ direction displacement.

Each thermal actuator's static actuation displacement was individually recorded by the top zoom camera microscope under two actuation conditions of $10 \mathrm{~V}$ and $15 \mathrm{~V}$, corresponding to $44 \%$ and $61 \%$ of PWM duty cycle, while the rest of the actuators were not powered. Resting and actuated positions of both center shuttle and the end-effector were recorded with blue lines in the captured pictures, as shown in Figure 4-6. The pixel to length ratio was then calibrated and displacement was acquired by measuring the pixel distance from captured frames. Figure 4-6 shows an example of displacement measurement for thermal actuator from top (a) and end-effector from side (b) and top (c).

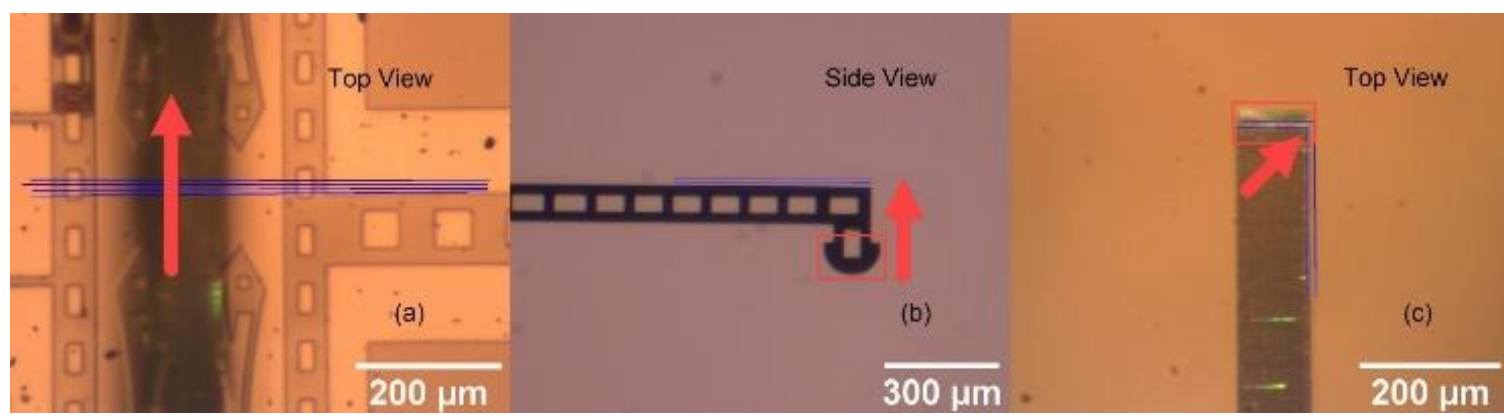

Figure 4-6 End-effector displacement experiment result. (a) Front actuator displacement when actuator $C$ was powered. (b) Side view of the end-effector displacement when $C$ was powered. (c) Top view of the end-effector displacement when $C$ was powered. The red box indicates the vision matching template in $(b)$ and $(c)$. Blue lines mark displacement in $(a)-(c)$.

The displacement data was imported into the FEA model to obtain simulated tip displacements for validation that are shown in Table IV rows 1 to 4 . Table IV rows 5 to 7 summarizes the measured tip displacements. The experimental results and the simulations agree for the sAFAM tip displacement in $\mathrm{X}$ and $\mathrm{Y}$ directions, but a much larger discrepancy for $\mathrm{Z}$ direction, even though the trends are the same. The disagreement could be because of etching errors in the two spring geometries. Another cause could have been due to the 
non-rigid Zyvex connector assembly, which can reduce the transmission effect of forces from the actuators to the end-effector. As a result, the coupling of the sAFAM's arm with actuators is less responsive than indicated in simulation. Nevertheless, the experimental results show that significant displacement in the $\mathrm{Z}$ direction can be achieved if actuators $\mathrm{A}$ and $\mathrm{C}$ are energized to $15 \mathrm{~V}$, as shown in Table IV.

\subsubsection{Workspace Measurement of sAFAM}

A feature recognition LabVIEW® VI was implemented to automate the end-effector workspace characterization using the top and side-view microscopes. Two recognizable features from the end-effector were selected to serve as the top and side visual matching templates as shown in Figure 4-6 (b), (c): the half circle feature on the end-effector for the side, and a bright edge of the end-effector that has clear visibility as seen from the top. The LabVIEW® PWM electronics interface was then combined with feature recognition VI to form an automated workspace measurement VI to determining the workspace of sAFAM. The VI cycles through four actuation voltages with PWM duty cycles of $0 \%, 31 \%, 63 \%$ and $98 \%$ applied to actuators A, B, C, D of the sAFAM to generate a total of $4^{4}=256$ sets of X-Y-Z coordinate measurements of the end-effector position. These 256 data points were recorded from both top and side view cameras as pixel count, and micron-pixel relation was estimated from actual measurements of similar features against a known etalon length to $0.546 \mu \mathrm{m} /$ pixel for top view camera and $0.500 \mu \mathrm{m} /$ pixel for side view camera. 


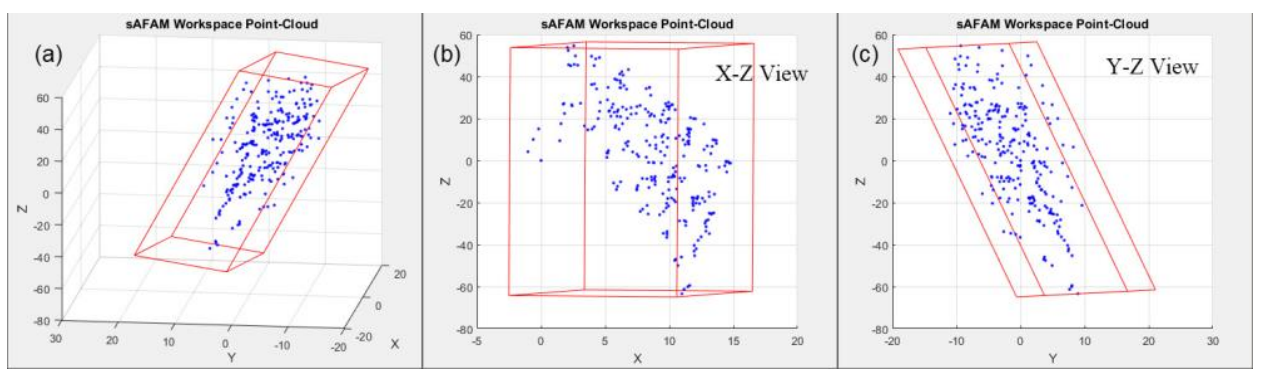

Figure 4-7 Measured SAFAM workspace (a) iso view, (b) X-Z plane projection (c) X-Y plane projection.

Then, a MATLAB ${ }^{\circledR}$ script was written to process and display the end-effector's displacement, which is calculated by subtracting the resting position from actuated positions and converting the pixel count to $\mu \mathrm{m}$. Eventually, a point-cloud representation of the sAFAM's workspace was visualized in Figure 4-7. The dimension of the workspace is bound by a 3D parallelepiped with approximate dimensions of $16 \mu \mathrm{m} \times 20 \mu \mathrm{m} \times 118 \mu \mathrm{m}$. The bounding box is an overestimate of the actual workspace of the end-effector, since most of the point clouds concentrates at the center and upper half of the boundary.

\subsection{Experimental Results: Microrobot Resolution and Repeatability}

Important specification of the tools for micro/nano manipulation applications are precision metrics, such as resolution, and repeatability.

To evaluate resolution and repeatability of the SAFAM, a series of measurements were conducted using the experimental setup shown in Figure 4-8 (a), (b). Precise measurements of the sAFAM's tip were conducted with the help of the laser displacement sensor - LKH008 (Keyence Corporation of America, NJ, USA) with $10 \mathrm{~nm}$ measurement resolution. A packaged sAFAM was mounted on the X-Y stage (manual/motorized) and a microscope with camera (PL-B742F, Pixelink, ON, CA) with the top or side view, allowing precise 
alignment of the LK-H008 and sAFAM's arm. While the LK-H008 was attached to a separate manual $\mathrm{X}-\mathrm{Y}-\mathrm{Z}$ stage independent from the sample stage.
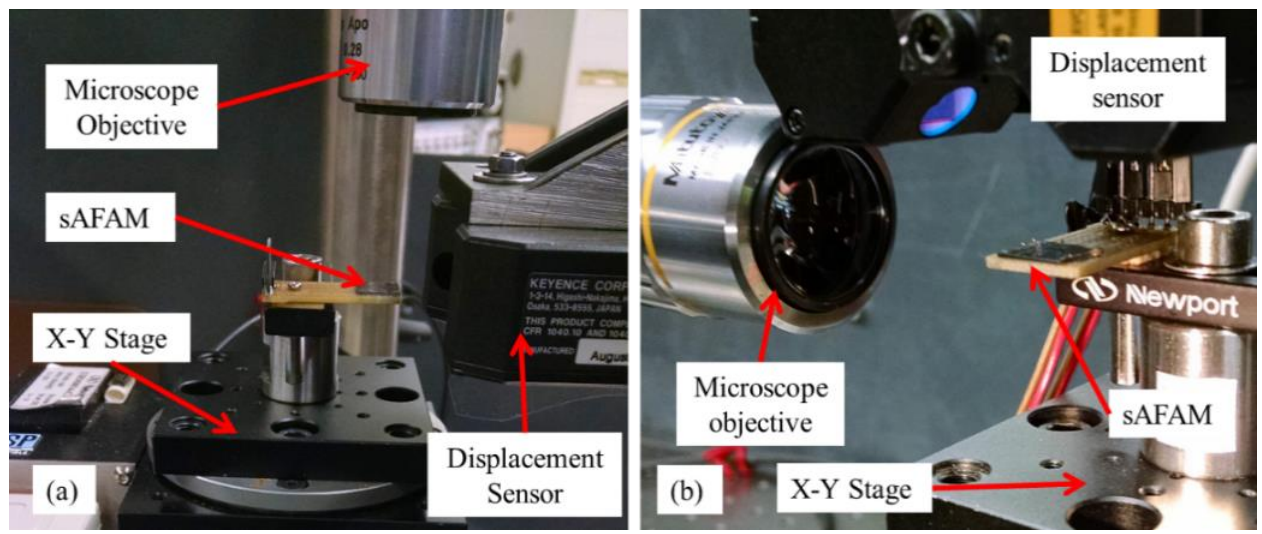

(c)
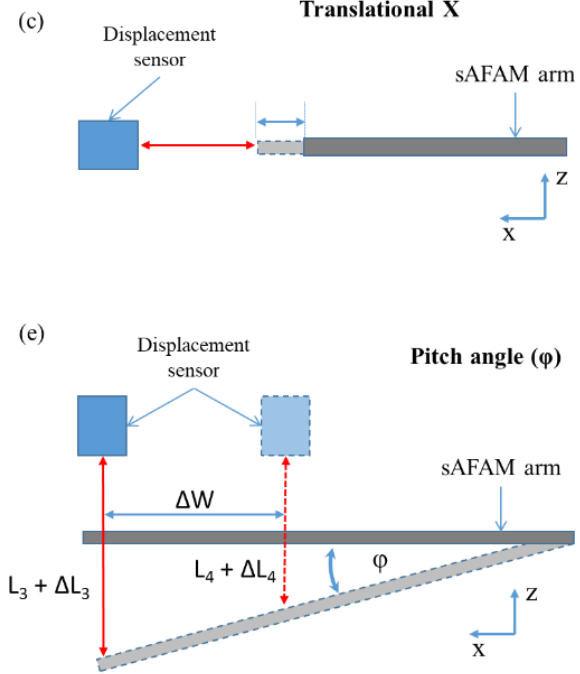

(d)
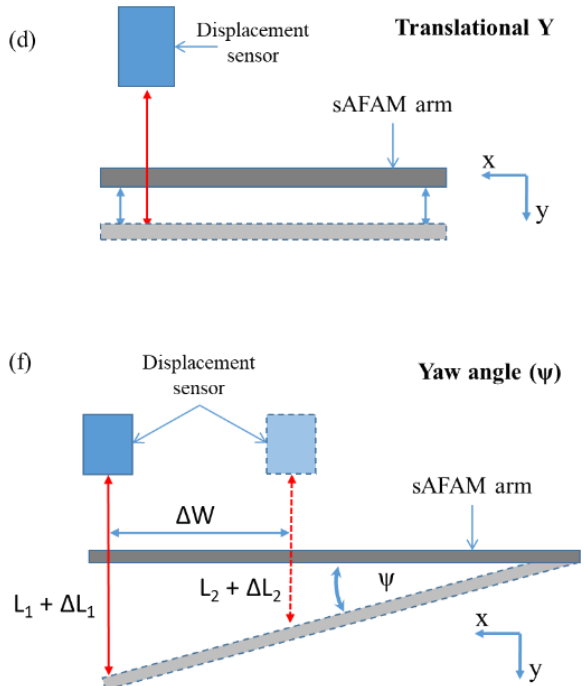

Figure 4-8 Images of two different arrangements of the experimental setup for the measurements of: a) translational motion in $X$ direction, the displacement sensor faces the end-effector tip and the camera observes from atop, and b) pitch angle $(\varphi)$ measurement, the displacement sensor mounts atop, and the camera observes from side. Schematics of 4 different arrangements for the measurements of: c) translational displacement in X direction, d) transl. displ. in Y direction, e) pitch angle $(\varphi), f)$ yaw angle $(\psi)$.

All measurements were taken with one sensor using different arrangements of the experimental set up depending on the considered direction of the sAFAM's arm displacement (Figure 4-8). Detection of the translational displacement along the $\mathrm{X}$ and $\mathrm{Y}$ axes (prismatic DOF (X, Y), Figure 4-8 (c), (d)), required that the measurements (with the 
help of displacement sensor) be taken along the corresponding directions of the arm's motion (X or $\mathrm{Y}$ ). Two series of the displacement measurements at two different positions relative to the SAFAMS's arm (Figure 4-8 (e), (f)) were conducted to measure revolute DOF (pitch angle $(\varphi)-X Z$ plane, yaw angle $(\psi)-X Y$ plane). In the case of yaw angle, the first series of measurements was taken at one position (L1), and after that, the sensor was moved along sAFAM's arm axis to a different position (L2) in the same plane of revolution (XY). The second series of measurements was taken, for the respective value of the actuating voltage (Figure 4-8 (f)). Using the collected data, yaw and pitch angles could be calculated from the following formula [41]:

$$
\psi \operatorname{or} \varphi=\arctan \left(\frac{\Delta L_{1}-\Delta L_{2}}{\Delta W}\right)
$$

Where $\Delta \mathrm{W}=\mathrm{W}_{1}-\mathrm{W}_{2}$, is the separation distance between two different positions of the sensor, whereas $\Delta \mathrm{L} 1$ and $\Delta \mathrm{L} 2$ represent incremental displacement detected by the sensor for the corresponding positions, L1 and L2. Chapter 4.4 describes the displacement measurements for the detection of the translational motion in $\mathrm{X}$ direction. To achieve this motion the pair of $\mathrm{A}+\mathrm{C}$ actuators were used for the $\mathrm{X}$ direction, and $\mathrm{B}$ and $\mathrm{D}$ were used for the $\mathrm{Y}$ direction. Actuator A was used in case of the pitch angle $\varphi$, and actuator B for yaw angle $\psi$. All the experiments were conducted under similar conditions, and actuation was realized using constant voltage or modulated at $4 \mathrm{~Hz}(50 \%$ duty cycle). The experiment used a DC power supply (6622A, Hewlett Packard, Palo Alto, CA), a function generator (33120, Agilent/Hewlett Packard, Palo Alto, CA) and a power amplifier (2348, TEGAM Inc., OH, USA). Displacement measurements were done at $200 \mu$ s sampling rate with LK-H008 displacement sensor, which allows collection of at least 1000 data points during actuation with input signal at $4 \mathrm{~Hz}$. 


\subsubsection{Resolution measurements}

Resolution is defined as the smallest increment of movement that sAFAM's arm can make. To determine such minimal measurable deflection, a series of experiments were conducted with the help of the displacement sensor, where the voltage input to the actuators was incremented with the step size between $5 \mathrm{mV}$ and $200 \mathrm{mV}$ for the given displacement of the end-effector, for each of the 4 degrees of freedom. Based on the sAFAM's arm response, it was determined that the values of the minimum voltage increment varied depending on the actuator, but the overall input needed induce detectable deflection of the robot's arm lay in the range of $50 \mathrm{mv}-200 \mathrm{mV}$. Such minimum increment value varied depending on the actuator, and the amount of actuation voltage needed to induce specific displacement of the arm.

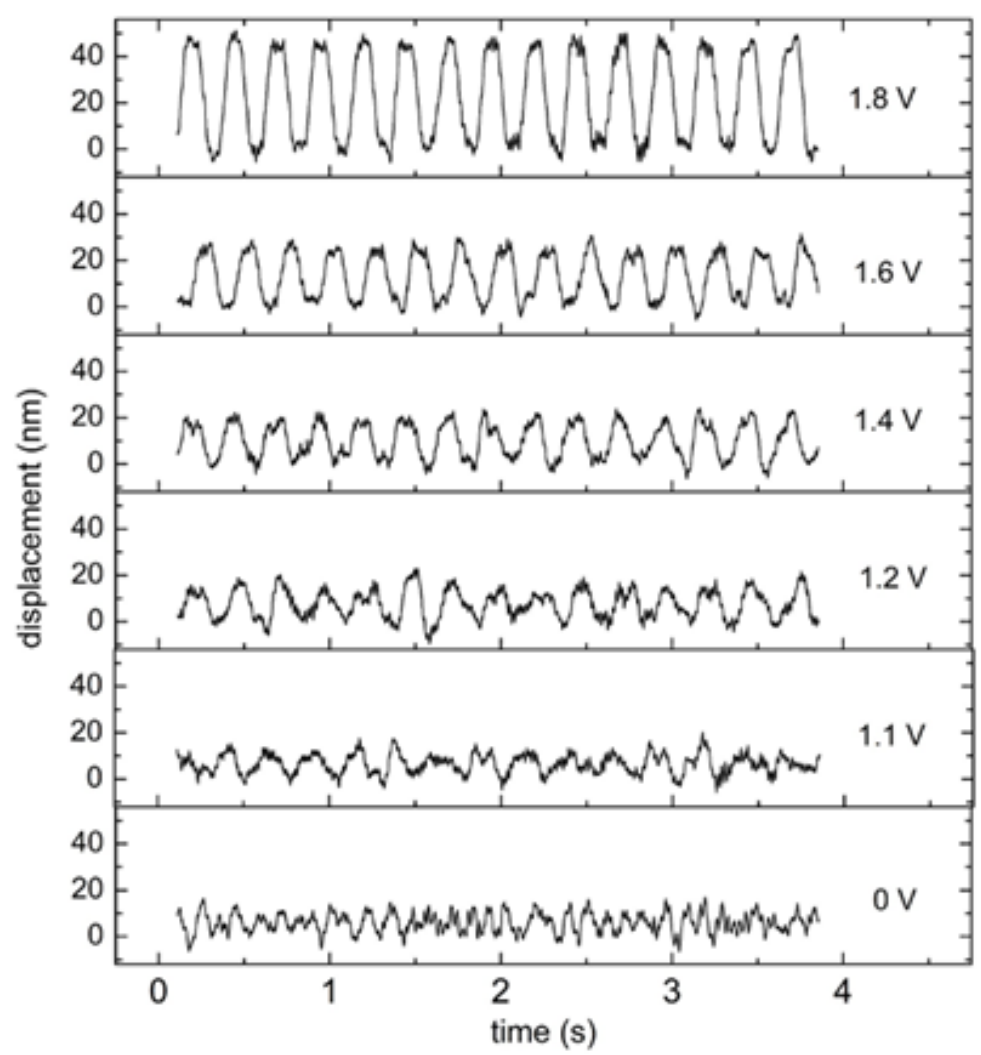

Figure 4-9 Displacement of the sAFAM arm in the $X$ direction (translation) as a function of time and actuation voltage. 
Figure 4-9 presents results of Keyence sensor measurements of the sAFAM's arm for cyclical displacement in direction $\mathrm{X}$. These plots are a qualitative illustration of the resolution determination, where it is evident that with the gradual increase of the voltage, deflection of the SAFAM arm is increasing. Here, the sAFAM arm was actuated with a modulated voltage signal (square wave at $4 \mathrm{~Hz}$ ) with amplitude value changing in small increments $(\sim 100-200 \mathrm{mV})$. Note that for actuation voltage of $1.8 \mathrm{~V}$, the deflection of the arm is about $50 \mathrm{~nm}$, and for $1.6 \mathrm{~V}$, around $25 \mathrm{~nm}$.
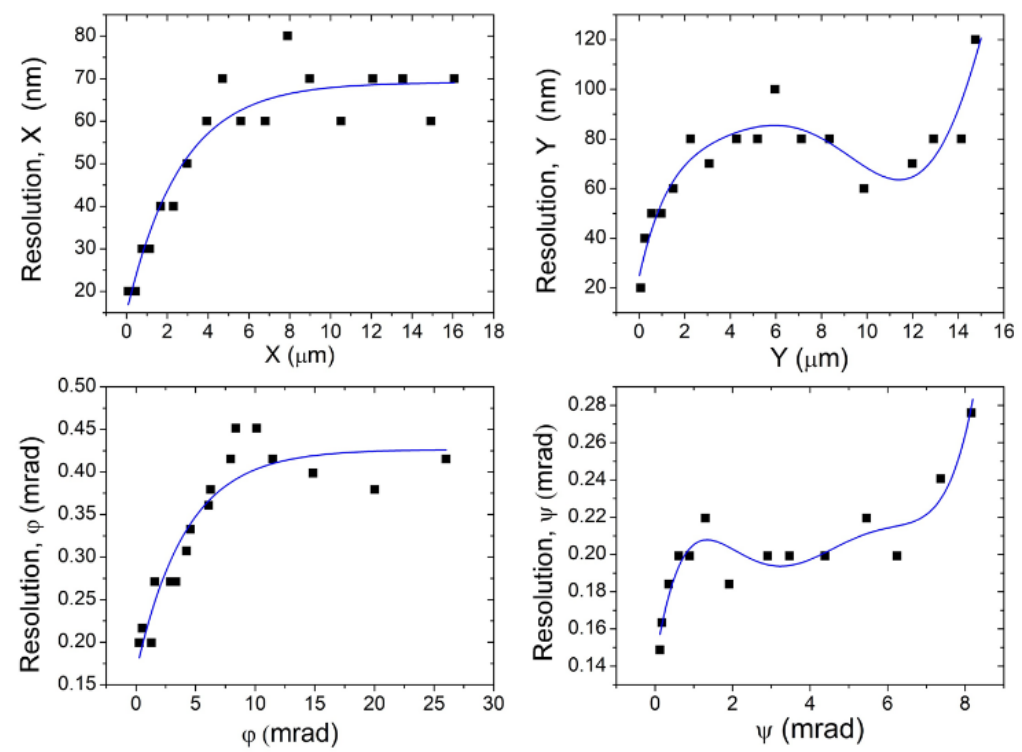

Figure 4-10 Resolution variation along four DOFs.

Figure 4-10 demonstrates variations of the resolution depending on the magnitude of sAFAM arm deflection. Resolution along the $\mathrm{X}$ and $\mathrm{Y}$ axes range between $20-120 \mathrm{~nm}$, and between $0.15-0.45 \mathrm{mrad}$ for the yaw and pitch angles. Resolution values for $\varphi$ and $\psi$ were calculated with the help of Equation 1. As can be seen from Figure 4-10, the resolution change for $\mathrm{X}$ and $\mathrm{Y}$ with increase of the displacement, follows a similar trend: A higher resolution and larger relative variation is achieved for smaller values of actuator inputs $(\mathrm{X}, \mathrm{Y}<6 \mu \mathrm{m})$. For displacements above $6 \mu \mathrm{m}$, there is a noticeable plateau where 
resolution remains relatively steady in a range between $60-80 \mathrm{~nm}$. As reported in [29], such behavior could be attributed to the nonlinear displacement and stiffness characteristics of the electrothermal MEMS actuator. Total variations in resolution for X and Y are $60 \mathrm{~nm}$ and $100 \mathrm{~nm}$, respectively. It should be noted that while in previous studies of the original AFAM's [29], resolution was lower for smaller deflections and improved with larger actuator inputs, we observed an opposite trend in this case. This discrepancy is likely due to the differences in the design of both structures, not a disagreement. It must be noted that the total variation in resolution measurements is $100 \mathrm{~nm}(\mathrm{Y})$ over $15 \mu \mathrm{m}$, which is $0.7 \%$ of maximum displacement (Figure 4-10).

\subsubsection{Repeatability Measurement Experiment}

Repeatability is the variation in the end-effector position over attempts to produce the same outcome under the similar experimental conditions, in our case displacing sAFAM's arm to the same position under the same actuator voltages (bias). Repeatability was determined by running number of actuation cycles at $4 \mathrm{~Hz}$ with a constant actuation voltage for a specific actuator, and for different magnitude of the deflection. This allows to determine changes in the initial and final positions of the robotic arm after each cycle. Repeatability is derived from error variance between the initial-final points for specific number of the cycles. Applying this method, it was determined that repeatability ranges between $20-150 \mathrm{~nm}$ for $\mathrm{X}$ and $\mathrm{Y}$ translation, and between $0.15-0.26 \operatorname{mrad}$ for $\varphi$ and $\psi$. 

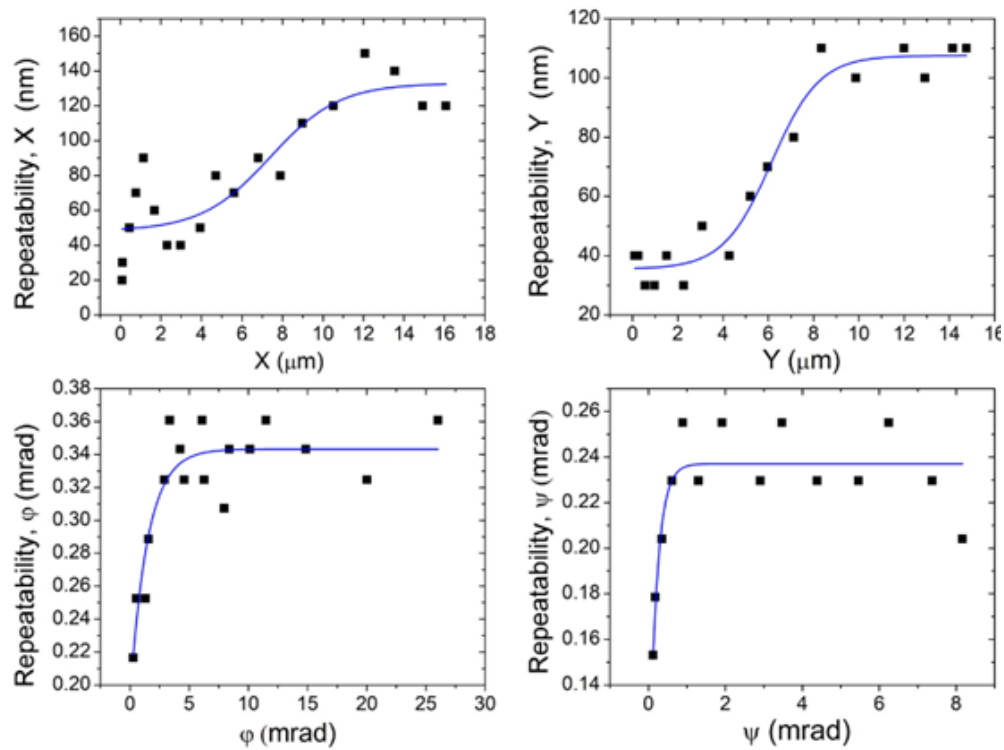

Figure 4-11 Repeatability variation along four DOFs.

Figure 4-11 illustrates variation of the repeatability depending on the deflection magnitude of the sAFAM arm in $\mathrm{X}, \mathrm{Y}$, pitch, and yaw. As in case of the resolution measurements, best for repeatability of the sAFAM structure is at lower magnitudes of deflection (actuation voltage). For the $\mathrm{X}$ and $\mathrm{Y}$ directions, there is a distinguishable plateau for the displacements above $8 \mu \mathrm{m}$, where repeatability ranges between $100-110 \mathrm{~nm}$ in $\mathrm{Y}$ direction and $110-150 \mathrm{~nm}$ in $\mathrm{X}$. That is the case for the pitch and yaw angle as well, where plateau is above $5 \mathrm{mrad}$ for pitch angle $(\varphi)$ and $1 \mathrm{mrad}$ for yaw angle $(\psi)$. 


\section{CHAPTER 5 THE SOLARPEDE MicROROBOT}

\subsection{Design of the SolarPede}

The SolarPede [43] [41] microrobot has two major components: an assembled MEMS die and an electronics backpack, with overall footprint of $26 \times 20 \times 6 \mathrm{~mm}$. The SolarPede is envisioned as a legged micro-crawler consisting of two subsystems, Electronic and MEMS, depicted in Figure 5-1. The first subsystem is a MEMS device which serves as the "chassis" and holds the actuated legs of the micro-crawler. The second subsystem is the electronic "backpack" implemented as a pair of printed circuit boards, solar panels and all electronic components necessary for the robot's powered operation.

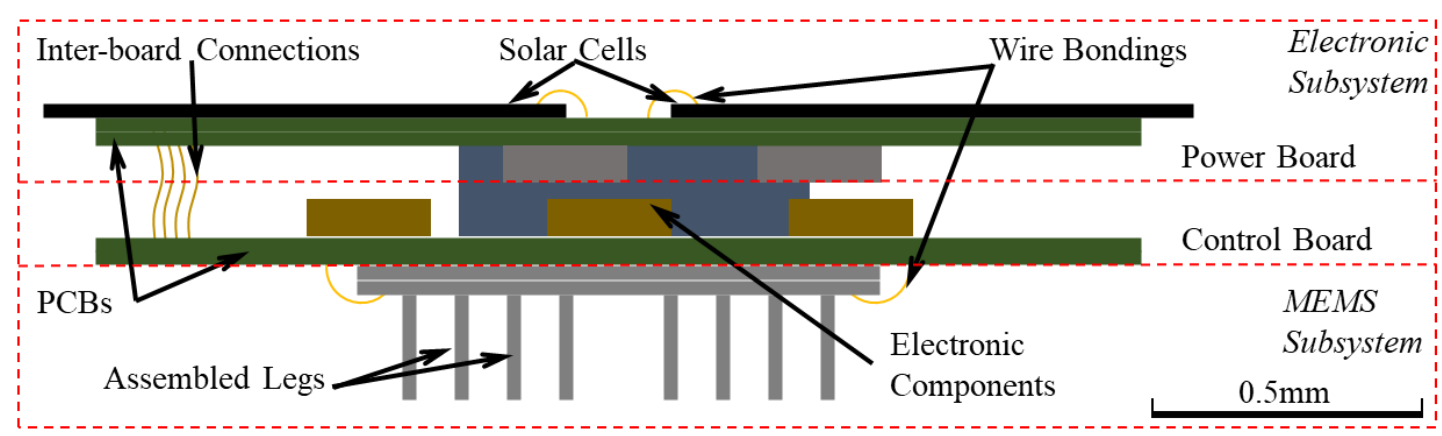

Figure 5-1 Side-view illustration of SolarPede's construction.

Figure 5-1 shows the major parts of SolarPede and demonstrates its construction, and a system level functionality abstraction is shown Figure 5-2. 


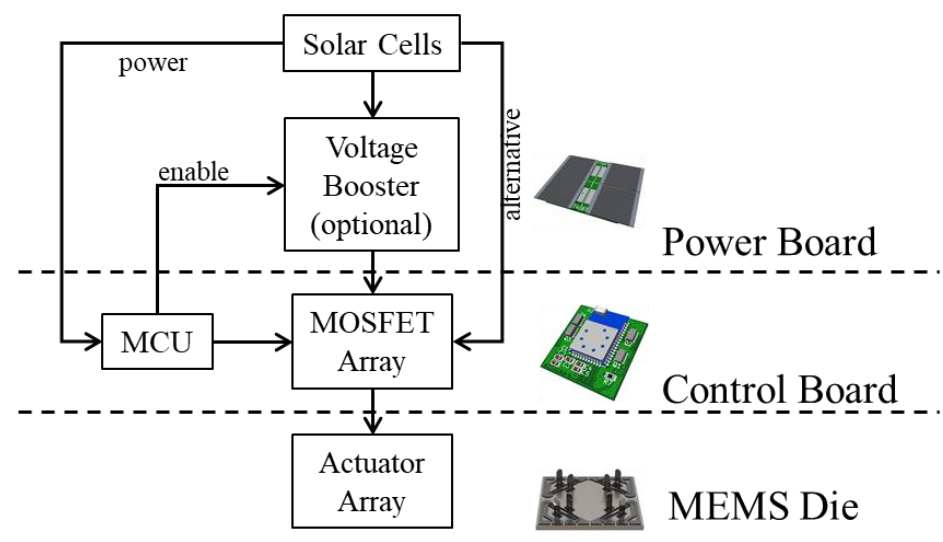

Figure 5-2 System level functionality abstraction of SolarPede.

\subsubsection{The MEMS Die and the Legs}

The "chassis" of SolarPede is a $1 \mathrm{~cm} 2$ MEMS die with 8 thermal actuators [112]. At the end of the thermal actuators' shaft, we included a so-called "Zyvex snap-fastener" [104] [107], which allows a vertical, compliant MEMS leg to assemble perpendicularly into the body of the robot. As indicated in Figure 5-3 (b), the Zyvex snap-fastener includes two components: a socket attached to the end of the shaft as a receptor and a plug on the leg. A special end-effector was used to pick up the leg and assemble it into the socket. The electrical interconnects for these actuators are positioned on two edges of the die so that wire bonding can be easily accomplished. The actuator-leg arrangement is shown in Figure 5-3 (a), with dimensions chosen to accommodate the Zyvex socket dimensions, and to guarantee significant leg displacements of up to $50 \mu \mathrm{m}$. The tip of the leg was rounded in order to mitigate errors in the event of a poor assembly resulting in a tilted leg. If the leg shape was rectangular, the corner of a tilted leg would create undesired off-axis actuation forces and prevent other legs from contacting the operating surface. 


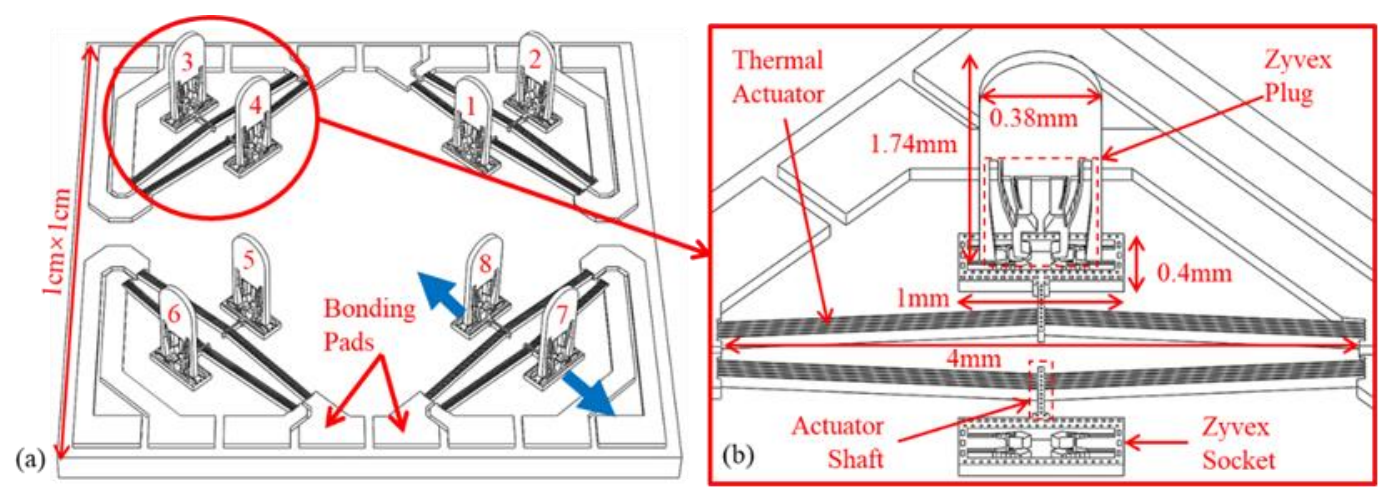

Figure 5-3 SolarPede's MEMS design. (a) CAD Model of assembled SolarPede die. (b) Dimension of the leg and thermal actuator, each actuator beam is $10 \mu \mathrm{m}$ wide and all assembled parts are $100 \mu \mathrm{m}$ thick.

Inspired by the mecanum wheels or differential drive arrangements for large mobile robots, we placed the 8 legs in a way that enables omnidirectional motion, as shown in Figure 5-3 (a). The die has been split into four identical quadrants; each contains two actuators with opposite diagonal direction of motions. Due to its symmetrical nature, omnidirectional motion can be achieved.

\subsubsection{Principle of Operation}

The SolarPede micro-crawler achieves locomotion using stick-and-slip effects between the legs and the operating surface, in a manner thoroughly discussed in [42]. By timing the displacement of the legs, a forward gait motion can be obtained for which the velocity is proportional to the amplitude-frequency product of the leg. Unlike the 8-legged nonholonomic design of the ARRIPede [85], the SolarPede contains actuator arrays which can be individually controlled to generate more dexterous maneuvers on the operating surface.

Actuating the eight legs in certain sequences, a combined force can be generated with controlled direction, hence creating directional motions. We use Figure 5-4 to explain three sequencing methods that create directional and steering motions, in which (a) to (d) 
illustrate the simple directional motions aligned with the $\mathrm{x}$ and $\mathrm{y}$ axes, (e) and (f) illustrate combined forces causing steering motions.

(1) To generate a combined force on the $+y$ direction as indicated by the red arrow in Figure 5-4 (a), actuators 1, 4, 6, 7 are engaged by applying an actuation voltage simultaneously. Then release them by removing the voltage, at the same time, engage actuator 2, 3, 5, 8. Black arrows are used to indicate the combined force for each quadrant. At the moment of the transition, all eight legs are in motion and causing a net force in the $+\mathrm{y}$ direction while the lateral directional $( \pm \mathrm{x})$ forces cancel each other.

(2) The second sequencing method is similar to (1) but relies on engaging half of the actuators at one time. For example, to generate forces in $+y$ direction, engage actuators 1 and 4, release, and engage 2 and 3. Then engage 6 and 7, release, and engage 5 and 8 . We will use this method as the preferred leg actuation sequence for our SolarPede, since our on-board power is limited.

(3) The third method engages each quadrant at one time: engage actuator 1, release, and engage 2; engage 4, release, then engage 3 ; engage 6 , release, then engage 5 ; engage 7 , release, then engage 8 , which generates force on $+y$ direction. This method was employed during our early prototype stage [113].

In addition to these methods, engaging one quadrant more often than others could generate steering motion, as indicated in Figure 5-4 (e) and (f).

Theoretically, all three methods can be used to create omnidirectional motions. The first method has the highest power requirement since it engages 4 actuators simultaneously, the second method engages two actuators simultaneously and the third engages one at each 
time. Simulations were carried out in Chapter 5.3 for method (2), to verify that directions of motion indicated by the arrows.
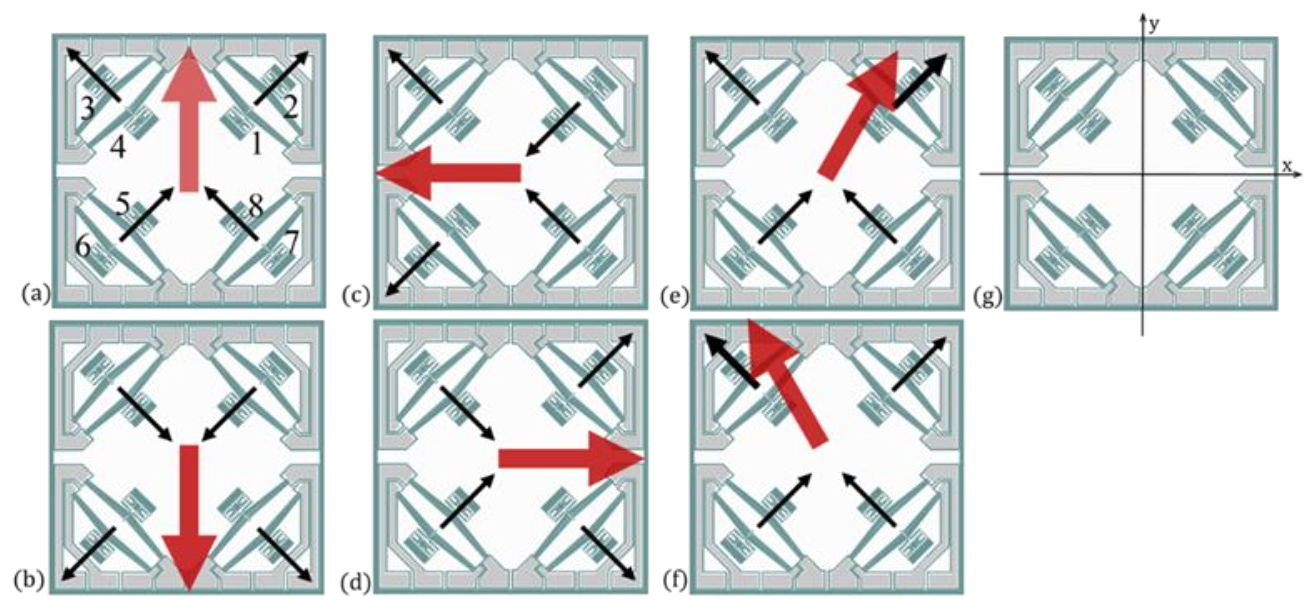

Figure 5-4 Theoretical vectors of holonomic motion methodology for SolarPede omnidirectional leg arrangement. Thicker vectors indicate motion performed at higher frequency than other vectors. (a) to (f) corresponding to up, down, left, right, up-right, and up-left forces. $(g)$ shows the definition of the $x$ and $y$ axes.

\subsubsection{Electronics and Solar Cells}

Powering and controlling of the SolarPede is accomplished through an electronic backpack mounted onto the Silicon die. The backpack contains a power board and a control board, as depicted in Figure 5-1. On top of SolarPede is the power board which accepts artificial light irradiation and converts energy for the microrobot. The power board has two major components: four solar cells in series configuration and a voltage boost converter circuit. The rated voltage of the solar cell is $2.5 \mathrm{~V}$ (Spectrolab®, CDO-100), four of them in series generate roughly $10 \mathrm{~V}$ under 5 suns irradiance, it is high enough to drive one thermal actuator to create observable displacement. Either used directly or boosted, this voltage is referred to as the "high voltage" in the remainder of this document. In addition, 
$2.5 \mathrm{~V}$ from the bottom of the four-in-series solar cells is wired out to provide power to the MCU.

Through wired connection, the high voltage, $2.5 \mathrm{~V}$, and ground are routed onto the control board, which contains a Bluetooth microcontroller unit (MCU) module, BL652 from Laird®, and a MOSFET (ON Semiconductor, NTHD4508N) array. The MCU works in conjunction with the MOSFET array to create the gaiting patterns required for steerable motion of the microrobot. And lastly, the MEMS die is attached to the back of the control board and wire bonded.

\subsection{MEMS Fabrication and Assembly}

\subsubsection{Fabrication}

The body and legs of SolarPede are fabricated on a silicon-on-insulator (SOI) wafer with the following parameters: the device layer is $\mathrm{N}$-type with 0.01 to $0.02 \Omega$-cm resistance, $100 \mu \mathrm{m}$ thick device layer and $<100>$ orientation; the buried oxide layer is $2 \mu \mathrm{m}$ thick and the handle layer is P-type with larger than $1000 \Omega-\mathrm{cm}$ resistance and $500 \mu \mathrm{m}$ thickness.

The fabrication follows the standard SOI process. An RCA clean was performed as the first step, to remove any organic, oxide and ionic contaminations; a thin layer of Chromium was sputtered with a PVD-75 sputter under DC mode with 300W, to boost Silicon-Gold adhesion. A thick layer of Gold for bonding points to the die carrier was sputtered under the same settings with longer sputtering time. Later, the metal was patterned with first photolithography and then etched by Gold etch and Chromium etch consecutively.

Deep reactive ion etching (DRIE) was performed with a strong photoresist mask (SPR220-3.0) on the wafer to define the body of the SolarPede. Etching time is derived from experience with the tool and checked under microscope to confirm finishing of 
etching. Before dicing the wafer, a protective layer of photoresist was spun to prevent debris from damaging or jamming the moving features. After dicing, selected dies were submerged in $49 \%$ hydro-fluoric acid to release the moving parts from the wafer surface. Eventually, all released dies were dried by critical point dryer (CPD) to conclude all fabrication processes.

\subsubsection{Assembly}

To assemble the legs on the SolarPede base, a custom microassembly system, called NeXus [106], was employed. The NeXus includes two manipulators (M2 holding a microgripper and M1 holding the MEMS die substrate) and three imaging systems as shown in Figure 5-5 (a). M1 consists of two motorized linear stages and one motorized rotation stage to realize X-Y-Ө three degrees of freedom (DOFs). M2 consists of motorized X-Y-Z-Ө stages and an extra manual X-Y stage to present 6 DOFs [73].

An end-effector mounted with a micro-jammer is connected to the manual X-Y stage by kinematic base on M2. Figure 5-5 (a) also shows the three imaging systems that provide views for the assembly processing from vertical, horizonal and side directions. The $45^{\circ}$ arrangement of the legs adds a twist in the assembly process; hence the assembly sequence should be carefully planned to avoid conflictions and damages to the structure.

The legs were first picked up by the micro-jammer (M2), and then the M1 manipulator rotates the sample chuck by $45^{\circ}$ so that the leg is aligned with the assembly site. As shown in Figure 5-5 (b), the leg approaches the assembly site by the red arrow at the bottom, the first leg attaches to the inner site marked as 1 , then the second leg was picked up and attached to the outer site marked as 2. After the two legs were attached, UV sensitive epoxy 
was applied and cured at the joint to increase the mechanical strength. Third, by rotating the sample chuck $90^{\circ}$, this process can be repeated to assemble the next group of legs.

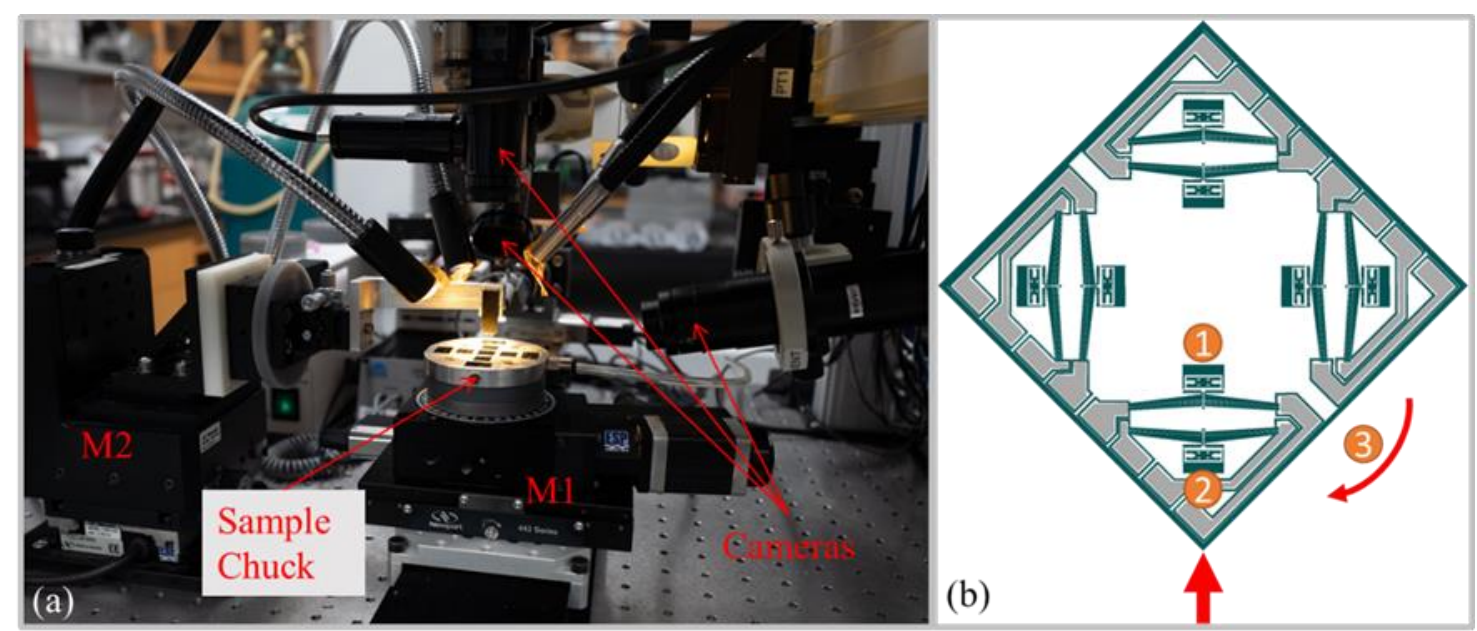

Figure 5-5 SolarPede's assembly. (a) NeXus microassembly station. (b) Leg assembly sequence: 1) Assemble the inner leg. 2) Assemble the outer leg. 3) Rotate the sample $90^{\circ}$ to assemble another pair of legs.

\subsection{Modeling and Analysis of the Microrobot}

In this section, a mathematical model of the microrobot is proposed, in order to analyze and further simulate the motion of the SolarPede prior to its design and fabrication. Simulations help to predict the expected velocity of the microrobot under various design parameters and constraints, including actuator sizes, friction coefficients and robot payloads. Later in this section, by using the model, we also discuss how unbalanced friction forces on the legs will likely affect the motion trajectory of SolarPede, and how the leg gating sequences will result in its motion along specific planar directions.

\subsubsection{Stick and Slip Model and Legs Displacement}

The SolarPede's motion is caused by breaking the static friction under its feet as well as at the joints in the heating cycle, followed by the whole robot body movement in the cooling cycle. Figure 5-6 depicts a lumped mass-spring-damper (MSD) model of the 
robot's leg. In this model, $\mathrm{M}_{1}$ and $\mathrm{M}_{\mathrm{b}}$ are the leg's mass and the robot body mass, respectively. $\mathrm{K}$ represents the stiffness and $\mathrm{B}$ is the damping coefficient of the thermal actuator.

In this model, two friction forces combined to resist motion inputs from the thermal actuator: one between the foot and operating surface, $\mathrm{f}_{1}$, and another at the joint between the assembled leg and the microrobot body, $\mathrm{f}_{2}$. Following Newton's second law of motion, the dynamic equations of such a system can be written as:

$$
\begin{gathered}
M_{l} \ddot{X}_{l}=-F_{a}+f_{1}+f_{2}+K\left(X_{b}-X_{l}\right)+B\left(\dot{X}_{b}-\dot{X}_{l}\right) \\
M_{b} \ddot{X}_{b}=F_{a}-f_{2}-K\left(X_{b}-X_{l}\right)-B\left(\dot{X}_{b}-\dot{X}_{l}\right) \\
f_{1}=\mu\left(M_{b}+M_{l}\right) g \\
f_{2}=\mu M_{b} g
\end{gathered}
$$

Where $\mu$ is the coulomb friction coefficient switching between static $\mu_{\mathrm{s}}$ and dynamic $\mu_{\mathrm{d}}$ in the stick-and-slip phases of leg motion, respectively, Fa is the actuation force generated by the thermal actuator under a certain input voltage of $\mathrm{V} . \mathrm{X}_{1}$ and $\mathrm{X}_{\mathrm{b}}$ are the corresponding displacements of $\mathrm{M}_{1}$ and $\mathrm{M}_{\mathrm{b}}$.

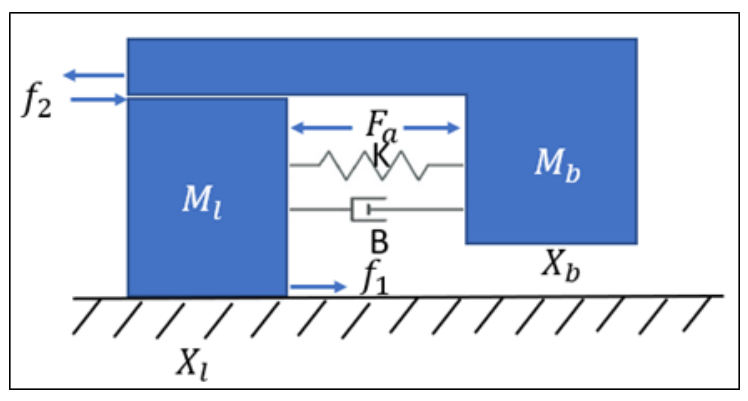

Figure 5-6 Mass-spring-damper model of the SolarPede's leg and body.

The value of $F_{a}$ during actuation cycles can be empirically estimated from steady-state displacement of the thermal actuator according to a first order model:

$$
F_{a}(V, f)=\frac{2 N A E \sin ^{2}(\theta)}{L} \cdot \frac{\lambda V^{2}}{1+s \frac{f}{f_{B W}}}
$$


In which $\mathrm{N}=6$ is the number of actuator beams within one thermal actuator, $\mathrm{E}$ is the Young's modulus of Silicon, $\theta$ is the actuator's beam bending angle, in our case $3.4^{\circ}$, A is the cross-section area of the actuator, $\mathrm{V}$ is the input voltage, $\mathrm{f}$ is the actuator's resonant frequency, $\mathrm{f}_{\mathrm{BW}}$ is the actuator's bandwidth, and $\lambda$ is an empirical constant [114].

Values used to simulate the leg motion were selected from the dimension parameters of the actuator and leg, in particular, $\mathrm{K}=185 \mathrm{~N} / \mathrm{m}, \mathrm{M}_{\mathrm{l}}=0.3 \mathrm{mg}, \mathrm{M}_{\mathrm{b}}=0.25 \mathrm{~g}$, while $\lambda$ and $\mathrm{f}_{\mathrm{BW}}$ were estimated from experiments as 0.03 and 50Hz. Furthermore, assuming the friction coefficients for Silicon-Silicon contact were $\mu_{\mathrm{s}}=0.4$, and $\mu_{\mathrm{d}}=0.33$. Our estimated forward velocity of the leg-body model is shown in Figure 5-7.
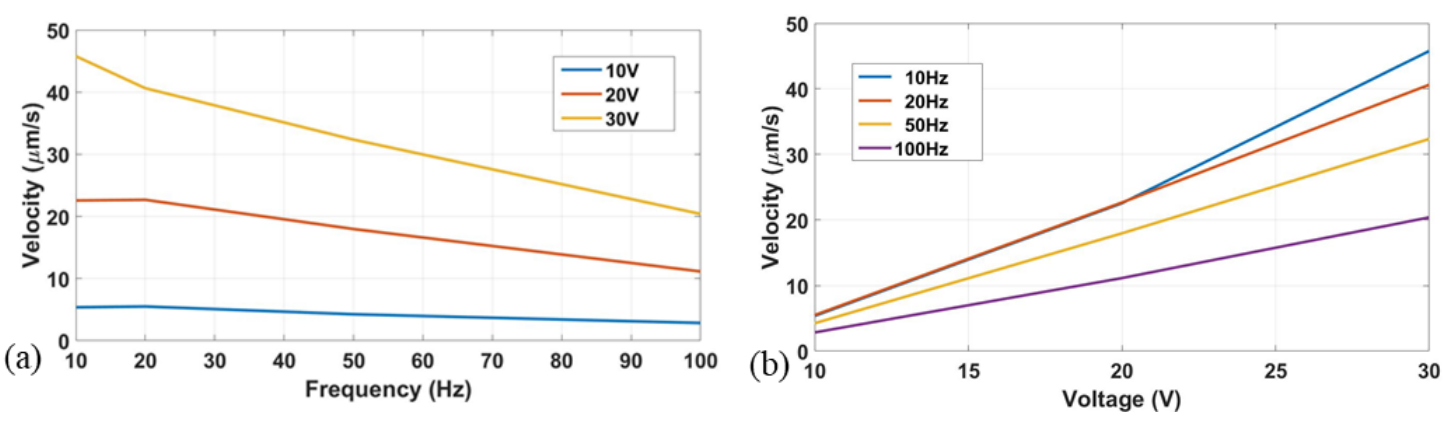

Figure 5-7 Plots of microrobot body velocity $\dot{X}_{b}$ vs. (a) leg actuation frequency, and (b) voltage.

In particular, simulation results indicate that we should expect forward velocities greater than $10 \mu \mathrm{m} / \mathrm{s}$ for a wide range of frequencies if the actuation voltage is $20 \mathrm{~V}$.

\subsubsection{Body Motion Analysis}

According to the 3 proposed gaiting patterns in Chapter 5.1.2, the SolarPede's holonomic motion relies on the combined directional force being applied to the body of the microrobot.

The SolarPede has eight legs in total. Each leg has one DOF, so that at least eight coupled differential equations are needed to fully describe its motion. To simplify this, we consider each quadrant as a single unit that generates a force Fx along the thermal actuator 
centerline, which in the ideal case passes through the SolarPede center of mass, as shown in Figure 5-8. Each force originates from the center of the two actuators, and they are described by the one-dimensional model we have derived. Notice that each of these Fx is a combined force. For example, $\mathrm{F}_{1}$ shown in Figure 5-8 is the force generated at the moment of actuator 1 retracking while actuator 2 is engaging. If this sequence reverses, the direction of $F_{1}$ also reverses.

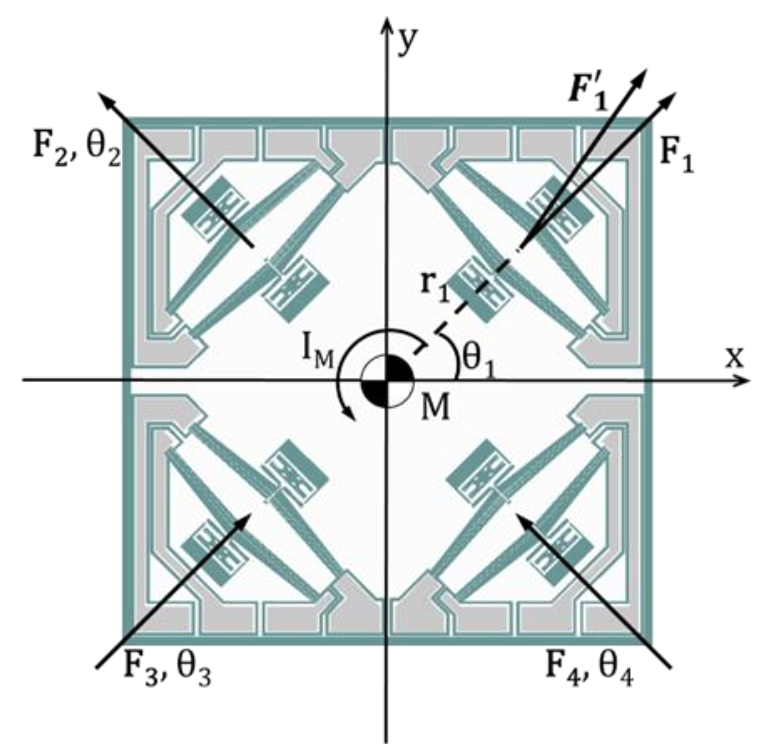

Figure 5-8 SolarPede body motion analysis.

The microrobot's body experiences a total force of:

$$
\sum \vec{F}=\overrightarrow{F_{1}}+\overrightarrow{F_{2}}+\overrightarrow{F_{3}}+\overrightarrow{F_{4}}
$$

From (6), if the magnitude of the four vectors are different, then directional motion can be imparted on the microrobot. Many factors contribute to this imbalance, including controllable effects (magnitude, frequency) and uncontrollable effects, for instance, different leg-surface contact condition, misalignment during leg assembly process, or tilted legs. 
Since the SolarPede actuator placement is symmetric, the actuation force can be seen directly applied at the geometric center in this model and hence can be combined into two, such as F1 and F2. By assigning different friction constants relating to F1 and F2, we can simulate the motion pattern when the legs experience different friction, as results shown in Figure 5-9 (a). In the plot, $\mu_{\mathrm{s}}$ and $\mu_{\mathrm{d}}$ represent static and dynamic friction constants respectively, and the 1 and $\mathrm{r}$ subscript represent force on the left or right.

As the simulation indicates, if the friction constant on the left and right are the same, a straight trajectory can be expected, otherwise sideway motion or drifting can be expected. The proposed gaiting method 2 described in Chapter 5.1.2 were simulated with our model and the resulting directions of motion are shown in Figure 5-9 (b). In this simulation, we combined the forces generated by actuators located in adjacent quarters to generate resultant forces $\mathrm{R}_{\mathrm{i}}, \mathrm{i}=1 \sim 4$. The simulation was run for 1 minutes, under $10 \mathrm{~Hz}$ and $30 \mathrm{~V}$. The maximum displacements were $2 \mathrm{~mm}$, indicating a velocity of $33.3 \mu \mathrm{m} / \mathrm{s}$. As expected, these resultant motions align with desired motion directions of the SolarPede, such as $\pm \mathrm{x}$ and $\pm \mathrm{y}$ and correspond to the direction of the arrows in Figure 5-4.
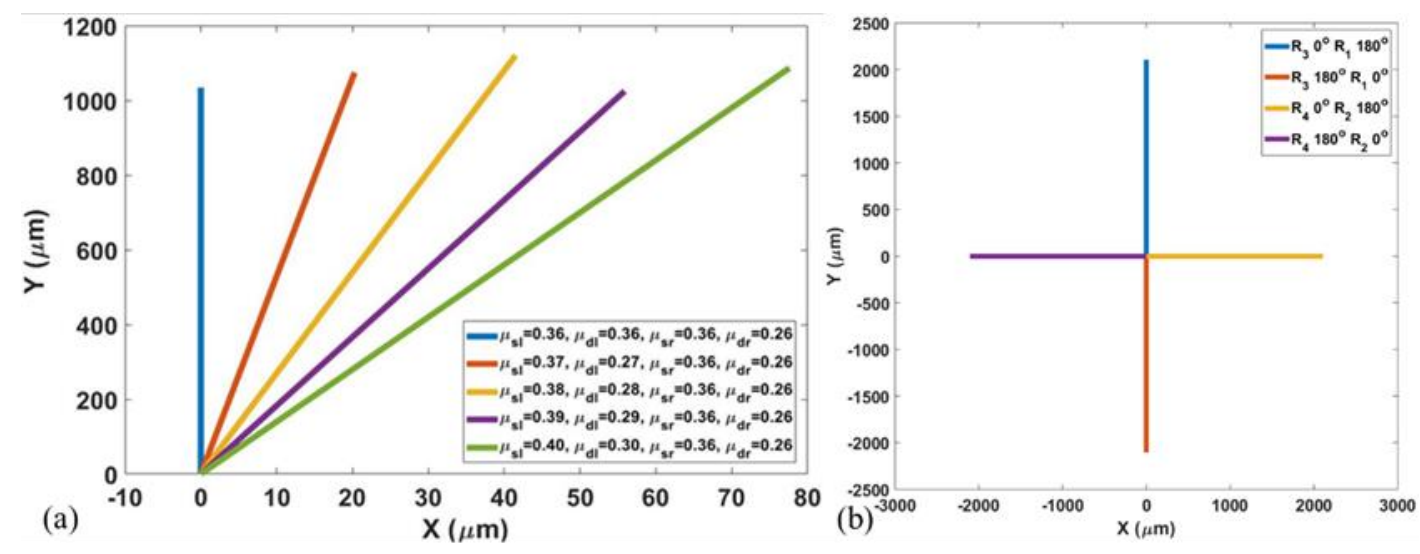

Figure 5-9 SolarPede's simulation result. (a) Result of SolarPede's motion trajectory simulation, with different friction constants on each leg. (b) Simulated trajectory of SolarPede's $2^{\text {nd }}$ gaiting method. $180^{\circ}$ phase shift between each combined actuation force. 
If we break the assumption of "all four forces align with the center of mass," the microrobot would spin. In this case, the extension of the force does not pass through the center of mass, such as $F_{1}^{\prime}$ in Figure 5-8. The distance from the center of the actuator to the center of mass denotes as rx. Then the total moment of the body can be written as:

$$
\sum M=I_{M} \ddot{\varphi}=\overrightarrow{r_{1}} \times \overrightarrow{F_{1}}+\overrightarrow{r_{2}} \times \overrightarrow{F_{2}}+\overrightarrow{r_{3}} \times \overrightarrow{F_{3}}+\overrightarrow{r_{4}} \times \overrightarrow{F_{4}}
$$

A moment IM would cause spinning motion of the microrobot. If the fabrication and assembly were precisely done, the microrobot should not experience any torque. But due to fabrication and assembly imperfections, spinning motion may be generated. This effect can be caused by different contact conditions between the leg and the operating surface, or by damaged actuator beams, causing the leg to move along different directions than designed. Spinning motion of the SolarPede is desired if the microrobot will be used as a positioner. This effect was observed experimentally, as discussed in Chapter 5.4.

\subsection{Experiment Result}

\subsubsection{Experiment Setup}

Three aspects of the proposed design have been evaluated in the following sections: power efficiency, control methodology, and wirelessly powered payload motion validation. Three stages of experiments were conducted to evaluate the design. First, we prototyped the design with bread boards to validate the control method and evaluate efficiency of the boost converter; second, we integrated all electronic components and the MEMS die into one large scale circuit board, $7 \mathrm{~cm}$ in size, in order to evaluate the overall design. This PCB is shown in Figure 5-10 (a). After this board was tested, we developed our first much smaller $2 \mathrm{~cm}$ size electronic backpack and wire bonded it to the MEMS die as shown in Figure 5-10 (c). 

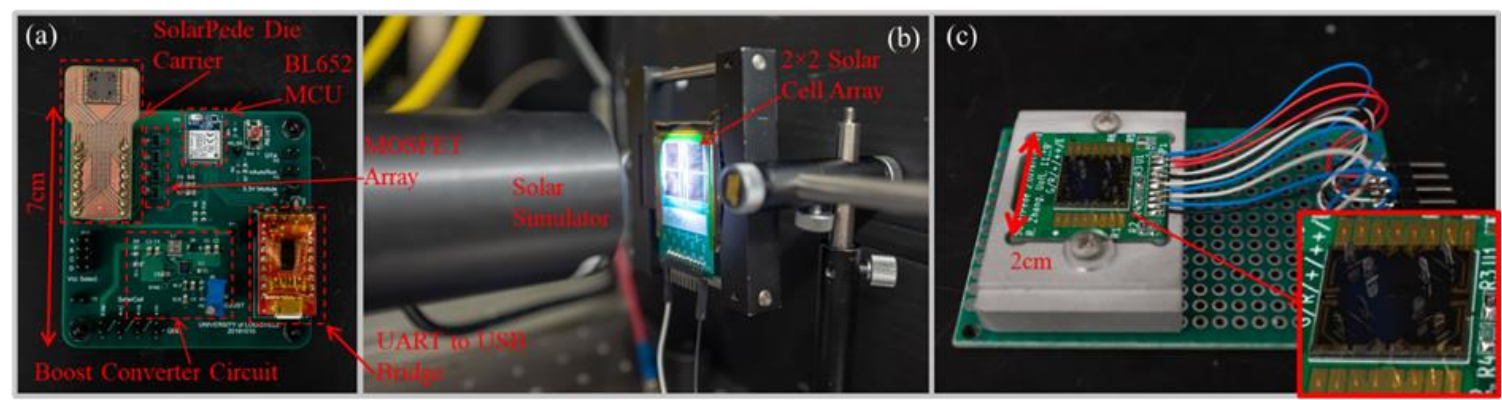

Figure 5-10 SolarPede experiment setup. (a) The $7 \mathrm{~cm}$ evaluation circuit. (b) Solar cell testing setup. (c) Millimeter-size prototype in a testing fixture.

The millimeter-size prototype validates the assembly of the SolarPede and evaluates the wireless powering capability. Components were selected from evaluation board experimental results. In this attempt to down-size the SolarPede, we mounted the solar cells on the power board shown in Figure 5-1. The voltage booster circuit was omitted due to efficiency consideration. The Bluetooth module, the MOSFET array, and the actuator die were all installed on the control board. Insulated thin copper wires from the transformer can be used to connect the power and control boards and they can be adhered to each other.

To assist the wire bonding process of the MEMS device, a customized aluminum fixture with a machined cavity was made to hold the control board, so that the wire bonder has a flat and stable working surface. This setup also allows all ports on the control board, including UART and power, to be interfaced to test its functionality separately, as shown in Figure 5-10 (c).

\subsubsection{Power Efficiency Evaluation}

Power is the most critical factor in SolarPede's electronics design, hence firstly evaluated. The power consumption of a single thermal actuator used in SolarPede was experimentally evaluated with stationary DC power supply. By swiping the voltage applied upon a single thermal actuator and measuring the current on the line, a power consumption 
curve was acquired, as shown in Table V. As indicated from the experiment, with $10 \mathrm{~V}$ input, the thermal actuator consumes roughly $260 \mathrm{~mW}$.

\section{Table V}

Power consumption of a single thermal actuator from 5 to $20 \mathrm{~V}$

\begin{tabular}{|l|c|c|c|c|c|c|c|c|c|c|c|c|c|c|c|c|}
\hline $\begin{array}{l}\text { Voltage } \\
(\text { V) }\end{array}$ & 5 & 6 & 7 & 8 & 9 & 10 & 11 & 12 & 13 & 14 & 15 & 16 & 17 & 18 & 19 & 20 \\
\hline $\begin{array}{l}\text { Current } \\
\text { (mA) }\end{array}$ & $\begin{array}{c}12 . \\
4\end{array}$ & $\begin{array}{c}14 . \\
5\end{array}$ & $\begin{array}{c}17 . \\
5\end{array}$ & $\begin{array}{c}20 . \\
7\end{array}$ & $\begin{array}{c}23 . \\
7\end{array}$ & $\begin{array}{c}26 . \\
0\end{array}$ & $\begin{array}{c}28 . \\
1\end{array}$ & $\begin{array}{c}30 . \\
2\end{array}$ & $\begin{array}{c}31 . \\
5\end{array}$ & $\begin{array}{c}33 . \\
2\end{array}$ & $\begin{array}{c}34 . \\
4\end{array}$ & $\begin{array}{c}35 . \\
7\end{array}$ & $\begin{array}{c}36 . \\
5\end{array}$ & $\begin{array}{c}37 . \\
1\end{array}$ & $\begin{array}{c}37 . \\
8\end{array}$ & $\begin{array}{c}38 . \\
4\end{array}$ \\
\hline $\begin{array}{l}\text { Power } \\
\text { (mW) }\end{array}$ & 62 & 87 & 123 & 166 & 213 & 260 & 309 & 362 & 410 & 465 & 516 & 571 & 621 & 668 & 718 & 768 \\
\hline
\end{tabular}

The solar cells used in our design must be small, highly efficient, and customizable,

which lead us to the solar concentrator products from SpectroLab. The model we sampled was $\mathrm{CDO}-100$ with $1 \mathrm{~cm}$ by $1 \mathrm{~cm}$ footprint. Four solar cells were assembled to a testing PCB, as shown in Figure 5-10 (b). Another advantage of using this type of solar cell is that they can utilize above 1000 suns of concentrated irradiance [115] with proper cooling scheme, which allows us to increase power output from solar cells by increasing solar simulator power output. In other words, power output does not saturate easily like other alternatives.

The efficiency of the boost converter was measured from a group of experiments. Preliminary experiments suggest that a single cell could provide $2.5 \mathrm{~V}, 75 \mathrm{~mW}$ power under 5 suns of irradiance. As a result, we chose to use four cells in series as the power source to the SolarPede, because total of 10V, 300mW can power one actuator, and their weight can be balanced when mounted on top of the microrobot.

A circuit shown in Figure 5-11 is used to determine the boost converter (TPS55340 from Texas Instruments $\left.{ }^{\circledR}\right)$ circuit efficiency. $\mathrm{V}$ and $\mathrm{A}$ are the output voltage and current measured directly from the solar cells, $\mathrm{R}$ is the load - a single thermal actuator, and A' and $V^{\prime}$ are current passed through the load and the voltage across the load. 


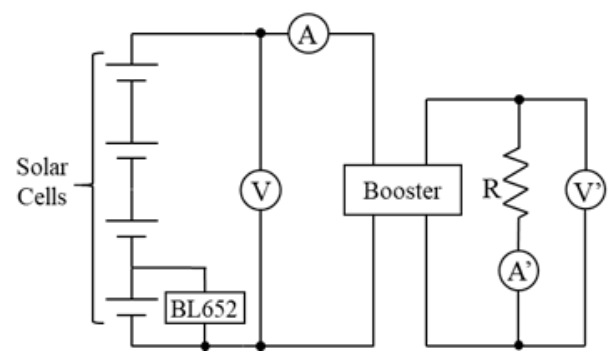

Figure 5-11 Solar cell and booster efficiency evaluation experiment circuit schematic.

The efficiency of the booster is determined by the following equation:

$$
\text { Boosting Efficiency }=\frac{\text { Load Power }}{\text { Solar Cell Power }}=\frac{V^{\prime} \cdot A^{\prime}}{V \cdot A}
$$

Two constraints apply to such an experiment: 1) The input voltage to the TPS55340 should not be below 2.9V. 2) If the output current A' cannot keep up with V', V' will drop below targeting voltage. For example, if we adjust the $\mathrm{V}$ ' to $16 \mathrm{~V}$, the thermal actuator requires $35.7 \mathrm{~mA}$ of current to maintain $16 \mathrm{~V}$ on the load. However, due to input power limitation, the boost converter cannot delivery $571 \mathrm{~mW}$ of power upon the load. At this moment, V' will drop below $10 \mathrm{~V}$, which indicates the boosting fails. According to experiments, a reliable boosting voltage was determined at around $11.5 \mathrm{~V}$. Under such condition, equation (8) can be evaluated by the following measurements:

$$
\text { Booster Efficiency }=\frac{V^{\prime} \cdot A^{\prime}}{V \cdot A}=\frac{11.2 \mathrm{~V} \times 30.6 \mathrm{~mA}}{10.0 \mathrm{~V} \times 45.1 \mathrm{~mA}} \approx \frac{342.7 \mathrm{~mW}}{451.0 \mathrm{~mW}} \approx 75.99 \%
$$

The efficiency of $75.99 \%$ matches datasheet specification. By using this configuration, $11.5 \mathrm{~V}$ can be reliably applied onto one actuator.

In order to use the limited power efficiently, we decided to remove the boost converter and apply $10 \mathrm{~V}$ directly on the actuators for the wirelessly powered operation, since $1.5 \mathrm{~V}$ voltage boost is not worth $24 \%$ power efficiency lost. This modification also reduces the weight of the backpack. 
The Bluetooth MCU BL652 module consumes maximum 24mW according to [113] [116], one tenth of a thermal actuator consumes, which can be easily covered by one solar cell and was confirmed by experiment.

\subsubsection{Control Method Validation}

The dynamic response of SolarPede's legs were investigated in [113], in which using a laser displacement sensor to validate the dynamic model in Chapter 5.3. Here, we conduct experiments to validate the functionality of our control methodology and circuit. The assembled SolarPede die was wire-bonded to a semiconductor carrier (Figure 5-12) and connected to the rest of the circuit through wires. A stationary DC power supply was used to provide $30 \mathrm{~V}$ to power the actuators. In this test, the SolarPede was in a "belly-up" configuration: all eight legs were facing upward, and a payload cut from a silicon wafer was placed directly onto the legs of SolarPede. This manner simulates the stick-and-slip motion of SolarPede without assembling the electronic backpack. To protect the integrity of the prototype, light payloads were used initially. The motion of the payload was recorded using a camera with $0.5,0.6$, and 1.2 grams of weight. A scratch was made on the surface of the silicon payload to assist displacement evaluation. The payloads were tested in succession, having their motion recorded before switching to the next payload, which were increasingly heavy. 


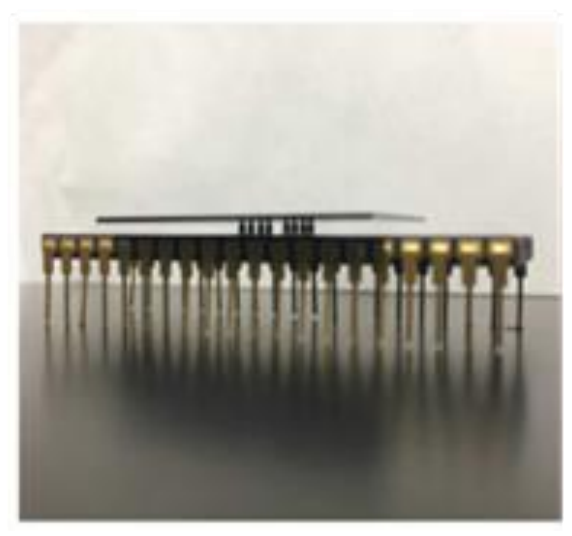

Figure 5-12 Assembled and packaged SolarPede die carrying a payload.

During experimentation, we decreased the duration of actuation pulse from $1 \mathrm{~s}$, which allowed the human eyes to easily perceive the leg's motion and helped to troubleshoot errors in the gaiting patterns. By decreasing pulse duration to $100 \mathrm{~ms}$, we observed that when each leg was actuated individually, the payload was not moving. If, however, the actuators were paired and moved in a synchronized manner, as described in method 3, the payload experiences a larger actuation force, and breaking static friction. In this manner, omnidirectional motion of the payload was achieved. The experimental results are presented in Table VI.

\section{Table VI}

Omnidirectional motion testing result

\begin{tabular}{|c|c|c|}
\hline Direction of motion & Observed Displacement (over 10 seconds) & Observed Velocity \\
\hline Up $(+\mathrm{y})$ & $155 \mu \mathrm{m}$ & $15.5 \mu \mathrm{m} / \mathrm{s}$ \\
\hline Down $(-\mathrm{y})$ & $121 \mu \mathrm{m}$ & $12.1 \mu \mathrm{m} / \mathrm{s}$ \\
\hline Left $(-\mathrm{x})$ & $133 \mu \mathrm{m}$ & $13.3 \mu \mathrm{m} / \mathrm{s}$ \\
\hline Right $(+\mathrm{x})$ & $113 \mu \mathrm{m}$ & $11.3 \mu \mathrm{m} / \mathrm{s}$ \\
\hline CW Rotation & - & $3 \mathrm{mrad} / \mathrm{s}$ \\
\hline
\end{tabular}

\subsection{4 “Belly-up” Payload Motion Testing Powered by Solar Simulator}

We tested our first millimeter-size prototype with the solar simulator (Newport 69907, 67005 and UXL-150S-O, Xe) to confirm that solar cell output is sufficient to drive the 
thermal actuators and hence move the payload under the "belly-up" configuration. In order to use the limited power from the solar cells efficiently, we can split the power to more than one actuator by $500 \mathrm{~Hz}$ pulse width modulation (PWM). On top of the PWM, an amplitude modulation (AM) defines individual actuation movement on each actuator.

However, this method only distributes power instead of generates more. According to our experiment, each thermal actuator consumes $260 \mathrm{~mW}$ of power under 10V DC. In order to use method 1 mentioned in Chapter 5.1.2, four actuators powered simultaneously, $4 \times 260 \mathrm{~mW}$ is required without PWM. If we use PWM with $50 \%$ duty cycle to power the four actuators, the power consumption can be reduced to $2 \times 260 \mathrm{~mW}$, at the cost of reduced displacement. Applying the same powering scheme, method 2 can be driven by $260 \mathrm{~mW}$ with PWM signal and reduced motion. While method 3 powers one actuator at one time so it consumes $260 \mathrm{~mW}$ without the need of PWM. On the other hand, if the solar cells cannot keep up with the power consumption of the thermal actuators, the voltage output from the cells will drop below the typical value. In this case, the solar cells are not able to drive the thermal actuators anymore and motion ceases.

In order to balance power consumption and drive more legs, we use the 2nd actuation method introduced in Chapter 5.1.2 to conduct the experiment, which requires engaging two actuators simultaneously, such as actuators number 1 and 4 . The two actuators can be powered by two PWM signals with 50\% duty cycle each and half-period phase shifting, demonstrated in Figure 5-13 (a). The next step of leg 2 and 3 are actuated by the same method. To generate force towards +y direction, the signal sequence shown in Figure 5-13 (b) was implemented. 


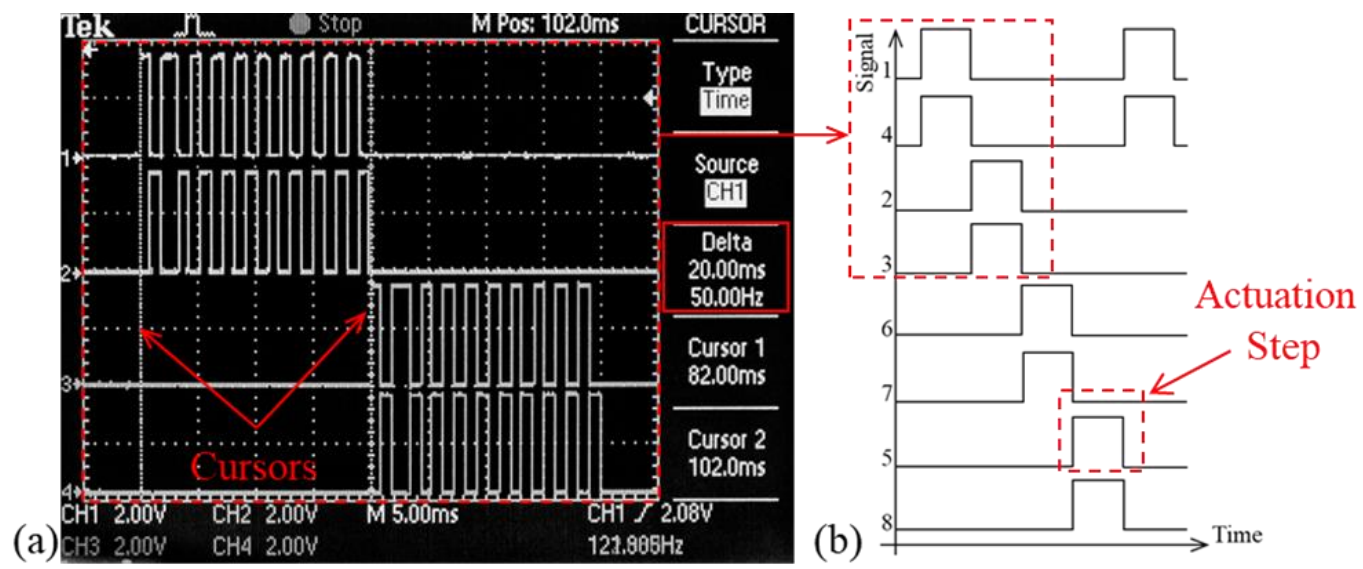

Figure 5-13 Leg actuation signal. (a) Signals of second actuation method on actuator \#1, \#4, \#2, and \#3: amplitude modulation (AM) on top of half-period, $180^{\circ}$ phase-shifted pulse-width modulation. Time between the two cursors is $20 \mathrm{~ms}$, the PWM signal is $50 \%$ duty cycle, $500 \mathrm{~Hz}$.

(b) Illustration of overall signaling for method 2.

In this way, two actuators can be engaged at the same time (equivalent of driving one actuator) at the expense of reduced displacement. According to [41], the product of actuation frequency (f) and amplitude (A) positively correlates to the velocity of the motion. In our experiment, the actuation voltage amplitude was determined by the solar cell's voltage and the PWM duty cycle, but we have control over the length of the "on" time on each actuator, which is referred as "actuation step" in the text, as indicated in Figure 5-13 (b).

In this experiment, the solar cells were exposed under 8 suns $(790 \mathrm{~mW} / \mathrm{cm} 2)$ of irradiance, and about $400 \mathrm{~mW}$ were measured from the solar cells. A piece of single-sidepolished Silicon die weigh $0.1 \mathrm{~g}$ was used as the payload, by applying sequence from method 2, displacement of the payload over 30s was recorded by a camera. Results are collected in Table VII. However, if we increase the payload to $0.4 \mathrm{~g}$ the motion ceases, possibly due to limited power. Therefore, in future refinements of the SolarPede design, 
the microrobot weight must decrease from $4 \mathrm{~g}$ to a much smaller number, the locomotion mechanism must be improved, or the light power must be boosted.

Table VII

Recorded payload displacement within 30s

\begin{tabular}{|c|c|c|c|c|c|c|}
\hline Actuation Frequency $(\mathbf{H z})$ & 100.0 & 71.4 & 50.0 & 25.0 & 16.7 & 12.5 \\
\hline Actuation Step Size (ms) & 5 & 7 & 10 & 20 & 30 & 40 \\
\hline Total Displacement $(\boldsymbol{\mu m})$ & 1160 & 1178 & 1307 & 698 & 484 & 440 \\
\hline Velocity $(\boldsymbol{\mu m} / \mathbf{s})$ & 38.67 & 39.27 & 43.57 & 23.27 & 16.13 & 14.67 \\
\hline
\end{tabular}

Figure 5-7 indicates that robot velocity is expected to increase if actuator voltage increases, and decreases, respectively, if the actuation frequency increases. With SolarPede, we are limited to around $10 \mathrm{~V}$ supply from the solar cells. As a result, the model predicts that velocity will be mostly constant between 5 and $10 \mu \mathrm{m} / \mathrm{s}$ as we sweep leg actuation frequencies from $10 \mathrm{~Hz}$ to $100 \mathrm{~Hz}$. Experiments suggest that while this trend may be true at low frequencies, with recorded velocities around $15 \mu \mathrm{m} / \mathrm{s}$, we also observed that payload displacement was maximized at frequencies closer to $50 \mathrm{~Hz}$. Indeed, velocities closer to $40 \mu \mathrm{m} / \mathrm{s}$ were observed for frequencies below $100 \mathrm{~Hz}$, suggesting that higher velocities can be obtained at higher leg actuation frequencies. This effect is not captured by the dynamic model shown in Figure 5-7, possibly due to ignoring additional effects such as vertical leg and joint flexibility that may be resonating at higher frequencies. In our past work with ARRIPede [42], the robot velocity was correlated with the square of the amplitudefrequency product, and our current observations are consistent with that conclusion.

Figure 5-14 shows the payload's trajectory within 30s, in which the desired displacement direction was the $+\mathrm{Y}$ axis. However, an undesired lateral displacement along $\mathrm{X}$ axis occurred, which can be explained by unbalanced friction forces on the lateral 
directions. Furthermore, small tilts on the legs during assembly could cause uneven friction force distribution, further leading to the lateral motion of the microrobot.

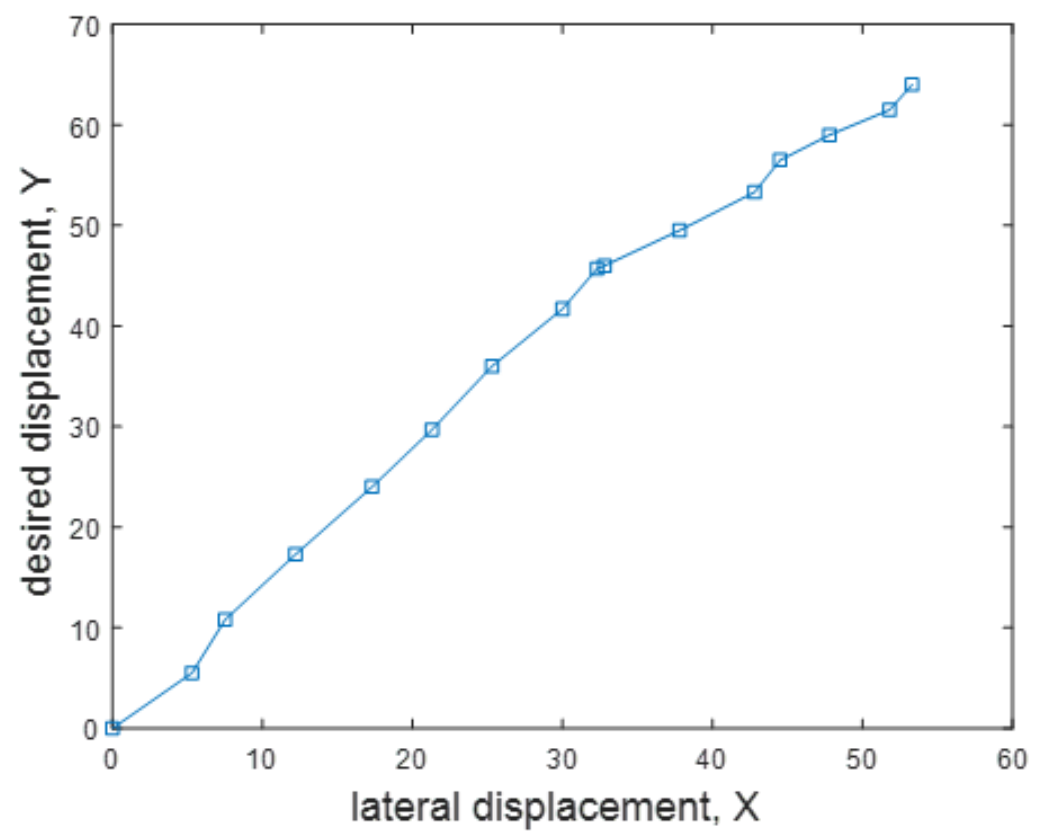

Figure 5-14 Trajectory of the payload displacement over 30s, with 7ms actuation step. 


\section{Chapter 6 CONCLUSION AND FUTURE WORK}

\subsection{Conclusion}

In this thesis, the microfactory concept was advanced through the introduction of 3 new types of microrobots described in detail in this document.

The ChevBot is the world's first micro-crawler robot powered by a laser beam. The microrobot concepts were experimentally validated under laser actuation can achieve fairly straight, uncontrolled velocities on a dry silicon environment in excess of 100 microns/s. Multi-physics analysis of ChevBot was conducted to study the opto-thermal-mechanical conversion of laser energy, and an experiment assist system identification of the thermal actuator under laser actuation was also conducted. Both models were validated against the experimental results and used to predict the locomotion velocity of ChevBot, in order to serve as a design tool. The stick-and-slip model was proposed to describe 1 dimensional motion of the ChevBot, which predicts ChevBot's velocity, given laser average power as input.

Using a custom-configured optical delivery and tracking experimental system, we collected experimental data of operation for both tethered and untethered microrobots. Results obtained in tethered operation can be utilized to fine tune the parameters of an opto-thermo-mechanical model for ChevBots, and thus use the models to improve future microrobot performance. By using a visual servoing tracking scheme, untethered microrobots were continuously powered to characterize their operation on a silicon 
substrate. The experiment confirms the pulsed laser signal is responsible for the gaiting motion of the ChevBot. Furthermore, the results suggest that velocities in excess of $100 \mu \mathrm{m} / \mathrm{s}$ can be obtained, and that the motion characteristics are influenced by substrate condition. The experimental results suggest that ChevBot can be used as a micro or nano scale positioner in microfactory applications.

The stationary microrobotics arm sAFAM is a novel 3D-assembled microrobot with four degrees of freedom and will be capable of pick-and-place delivery of nanocomponents. The microrobot was first simulated using Finite Element (FEA) analysis to dimensionally size the spring coupler design in the arm assembly. Simulation results indicated a workspace of $13 \mu \mathrm{m} \times 47 \mu \mathrm{m} \times 115 \mu \mathrm{m}$. The microrobot components were then fabricated on an SOI wafer with $100 \mu \mathrm{m}$ thick device layer, then released and assembled using a custom passive microgripper mounted on a robotic assembly station. The overall dimensions of the assembled SAFAM microrobot are $10 \mathrm{~mm}$ by $10 \mathrm{~mm}$ by $1.5 \mathrm{~mm}$, while its measured workspace was $16 \mu \mathrm{m} \times 20 \mu \mathrm{m} \times 118 \mu \mathrm{m}$. Thus, simulation results using Finite Elements are consistent with the experimental results obtained using stereo images acquired from a high-resolution microscope imaging system.

Precision evaluation of the proposed SAFAM structure with a laser displacement sensor further indicates that microrobot's best resolution is $20 \mathrm{~nm}$. Resolution coupled with the best result for the repeatability of 20nm, makes the sAFAM suitable for applications at submicron and nanoscale. Due to the fact that the sensor we employed to measure resolution had a precision of $10 \mathrm{~nm}$, we expect that resolution and repeatability of the SAFAM may be below this value. Furthermore, the measured decrease of resolution and repeatability at 
specific locations in the workspace could be due to nonlinear buckling effects in the thermal actuators, and amplification of fabrication errors on the two springs.

SolarPede is a novel light-powered micro-crawler, that can act as a mobile microrobot in "belly down" configuration, or as a substrate conveyor in its "belly up" configuration. The SolarPede's untethered operation was analyzed and experimentally validated in the thesis. The microrobot body was fabricated using DRIE process from SOI wafers with 1 $\mathrm{cm} 2$ footprint. 8 Silicon legs were assembled into compliant socket connectors using a custom microassembly station in our lab. The leg layout on the microrobot body ensured that omni-directional 2D motions can be achieved using stick-and-slip of the leg to the operating surface. An electronic backpack consisting of a Bluetooth enabled microcontroller, four solar cells, and power electronics was designed and connected to the packaged body of the microrobot. A stick-and-slip model was implemented to analyze leg displacement as well as expected force output. Further, the model was used to predict the microrobot's motion velocity and trajectory. The locomotion of the microrobot was studied in the "belly-up" or conveyor configuration by placing a payload on top of the inverted legs. Experiments were conducted to verify that the robot can achieve translational motions upon up-down and left-right directions; under solar cells powered trials, a maximum speed of $40 \mu \mathrm{m} / \mathrm{s}$ can be achieved with 8 suns of artificial light irradiation.

\subsection{Future Work}

In future work, we will conduct a more comprehensive study of the ChevBot's motion under various laser inputs and surface characteristic conditions. The minimum and maximum step size of ChevBot will also be examined under high speed camera. We will work on implementing closed loop controllers to steer (rotate, translate) the robot along 
controlled trajectories. Control over the robot trajectory can be accomplished by designing differential thermal responses in its feet and dimple, by changing the frequency of the laser, and by controlling the relative location of the laser beam and microrobot. Based on above improvements, we would include an AFM tip to the body of the ChevBot so that it can be demonstrated to interact with micro and nano objects. Further, we would like to demonstrate using multiple ChevBots to perform manipulation tasks such as nano assembly.

For sAFAM, the configuration of the in-plane stages needs to be revised on two directions. First, the serpentine springs that holding the shuttle need to be rearranged to levitate the $x-y$ motion coupling effect, so that the end-effector has cleaner motion. Second, the buckling effect on the thermal actuators needs to be addressed by improving fabrication precision, in order to provide higher output force on the end-effector when actuating voltage is high. Structural fatigue of an assembled SAFAM will be studied with repeated actuation tests to evaluate assembly and structural reliability. An increased Zyvex connector stiffness will be studied in conjunction with new bonding materials, and automation of the assembly processes is being pursued. The motion of the end-effector will be modeled so that a controller can be implemented. We will also conduct in-situ SEM characterization of the SAFAM, together with micro/nanoprobing and handling tasks. Figure 6-1 demonstrates an on-going effort of using sAFAM to perform micro/nano manipulation within SEM chamber. A sAFAM is wire bonded onto a supporting PCB that allows electrical connection to access each actuator. A customized 3D printed structure is used to support the assembled sample so that the end-effector can reach an operating 
surface. Furthermore, we will demonstrate multiple sAFAMs cooperate to perform manipulation tasks.

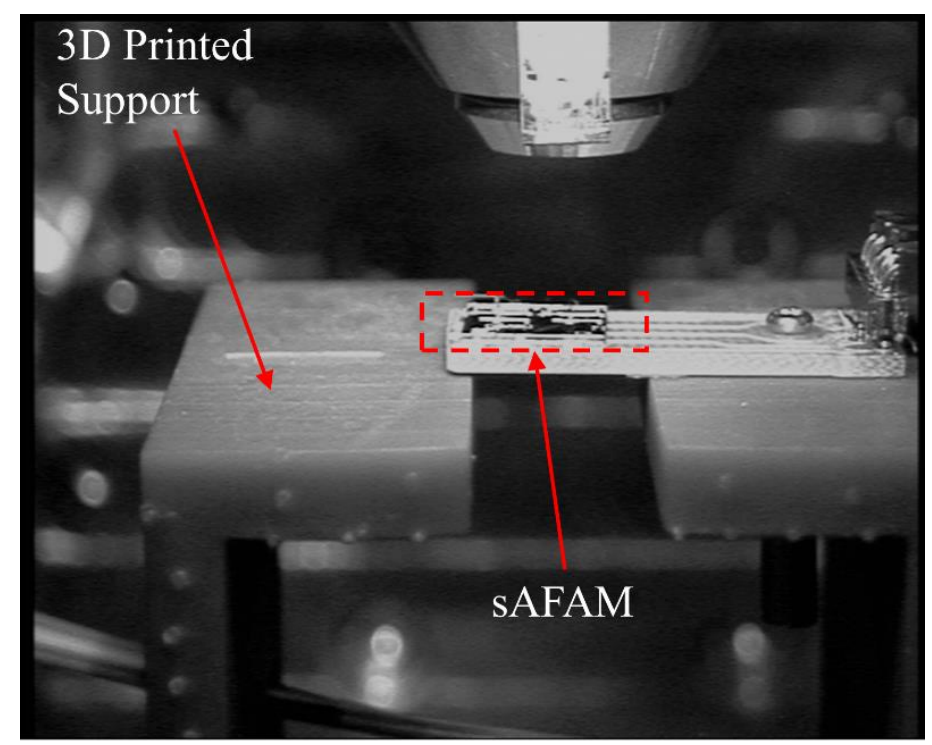

Figure 6-1 SAFAM with support in SEM chamber.

To finish the development of SolarPede microrobot, dynamic simulations will be conducted to optimize additional design aspects of the SolarPede. New leg designs, placement, and numbers will be investigated for handling of assembly errors, heavier payload, and traversing different surface materials and flatness conditions. The size and weight of the electronics backpack will be further decreased and SolarPede microrobot can be fully tested. Furthermore, closed-loop control of the planar position of the microrobot will be achieved using visual microscope feedback to affect the gait cycles in appropriate quadrants and correct for undesired drift motions. 


\section{REFERENCES}

[1] Wikipedia, "Robot," Wikimedia Foundation, Inc., 3 April 2020. [Online]. Available: https://en.wikipedia.org/wiki/Robot. [Accessed 9 April 2020].

[2] S. Zhang, "Fabrication of novel biomaterials through molecular self-assembly," Nature Biotechnologyvolume, no. 21, pp. 1171-1178, 2003.

[3] H. Shimoda, S. J. Oh, H. Z. Geng, R. J. Walker, X. B. Zhang, L. E. McNeil and O. Zhou, "Self-Assembly of Carbon Nanotubes," Advanced Materials, vol. 14, no. 12, pp. 899-901, 2002.

[4] H. T. Maune, S.-p. Han, R. D. Barish, M. Bockrath, W. A. Goddard III, P. W. K. Rothemund and E. Winfree, "Self-assembly of carbon nanotubes into twodimensional geometries using DNA origami templates," Nature Nanotechnology, no. 5, pp. 61-66, 2009.

[5] S. M. Felton, M. T. Tolley, C. D. Onal, D. Rus and R. J. Wood, "Robot SelfAssembly by Folding: A Printed Inchworm Robot," in IEEE International Conference on Robotics and Automation (ICRA), Karlsruhe, Germany, 2013.

[6] M. T. Tolley, S. M. Felton, S. Miyashita, D. Aukes, D. Rus and R. J. Wood, "Selffolding origami: shape memory composites activated by uniform heating," Smart Materials and Structures, vol. 23, 2014.

[7] V. Derenskyi, W. Gomulya, W. Talsma, J. M. Salazar-Rios, M. Fritsch, P. Nirmalraj, H. Riel, S. Allard, U. Scherf and M. A. Loi, "On-Chip Chemical SelfAssembly of Semiconducting Single-Walled Carbon Nanotubes (SWNTs): Toward Robust and Scale Invariant SWNTs Transistors," Advanced Materials, vol. 29, no. 23, 2017.

[8] J. Fang, K. F. Bohringer and H. Achour, "Self-Assembly," in Comprehensive Microsystems, Elsevier, 2008, pp. 403-429.

[9] Y. Qin, A. Brockett, Y. Ma, A. Razali and J. Zhao, "Micro-manufacturing: research, technology outcomes and development issues," The International Journal of Advanced Manufacturing Technology, no. 47, pp. 821-837, 2009.

[10] S. Mekid and S. Bashmal, "Engineering Manipulation at Nanoscale: Further functional specifications," Journal of Engineering Design and Technology, vol. 17, no. 3, pp. 572-590, 2019. 
[11] kleindiek nanotechnik, "Micromanipulator for Electron Microscopy," Kleindiek Nanotechnik GmbH, $25 \quad 32020 . \quad$ [Online]. Available: https://www.nanotechnik.com/mm3a-em.html. [Accessed 253 2020].

[12] Imina Technologies SA., "NANO - Robotics Solutions for Electron Microscopes," Imina Technologies SA., $25 \quad 32020 . \quad$ [Online]. Available: https://imina.ch/products/NANO-Nanoprobing-SEM-Solutions. [Accessed 253 2020].

[13] T. Tanikawa and T. Arai, "Development of a Micro-Manipulation System Having a Two-Fingered Micro-Hand," IEEE Transactions on Robotics and Automation, vol. 15, no. 1, pp. 152-162, 1999.

[14] Y. Higuchi, T. Kusakabe, T. Tanemura, K. Sugano, T. Tsuchiya and O. Tabata, "Manipulation system for nano/micro components integration via transportation and self-assembly," in IEEE 21st International Conference on Micro Electro Mechanical Systems, Wuhan, China, 2008.

[15] T. Fukuda, M. Nakajima, M. R. Ahmad, Y. Shen, K. Nogawa and M. Kojima, "Robotic manipulation and control for Micro and Nano mechatronics," in 10th IEEE International Conference on Nanotechnology, Seoul, South Korea, 2010.

[16] H. Rosen and T. Abribat, "The rise and rise of drug delivery," Nature Reviews Drug Discovery, vol. 4, p. 381-385, 2005.

[17] A. V. Singh and M. Sitti, "Targeted Drug Delivery and Imaging Using Mobile Milli/Microrobots: A Promising Future Towards Theranostic Pharmaceutical Design," Current Pharmaceutical Design, vol. 22, no. 11, pp. 1418 - 1428, 2016.

[18] D. Irimia, Encyclopedia of Microfluidics and Nanofluidics, Boston, MA: Springer, 2008.

[19] E. B. Steager, M. S. Sakar, C. Magee, M. Kennedy, A. Cowley and V. Kumar, "Automated biomanipulation of single cells using magnetic microrobots," The International Journal of Robotics Research, vol. 32, no. 3, pp. 346-359, 2013.

[20] A. Ichikawa, S. Sakuma, F. Arai and S. Akagi, "Untethered micro-robot with gripping mechanism for on-chip cell surgery utilizing outer magnetic force," in IEEE International Conference on Robotics and Automation (ICRA), Hong Kong, China, 2014.

[21] S. Kuindersma, R. Deits, M. Fallon, A. Valenzuela, H. Dai, F. Permenter, T. Koolen, P. Marion and R. Tedrake, "Optimization-based locomotion planning, 
estimation, and control design for the atlas humanoid robot," Autonomous Robots, no. 40, p. 429-455, 2015.

[22] "ATLAS®," Boston Dynamics, 9 April 2020. [Online]. Available: https://www.bostondynamics.com/atlas. [Accessed 9 April 2020].

[23] "German robotics and automation industry set to break records and grow faster than previously expected," Robotics and Automation News, 8 October 2017. [Online]. Available: https://roboticsandautomationnews.com/2017/10/08/german-roboticsand-automation-industry-set-to-break-records-and-grow-faster-than-previouslyexpected/14406/. [Accessed 9 April 2020].

[24] M. Hebert, "UofL's Popa is research co-leader on \$24 million partnership to advance next-gen manufacturing tech," UL News, 17 December 2019. [Online]. Available: https://www.uoflnews.com/section/science-and-tech/uofls-popa-isresearch-co-leader-on-24-million-partnership-to-advance-next-gen-manufacturingtech/. [Accessed 9 April 2020].

[25] "WALL-E GALLERY," The Walt Disney Company, [Online]. Available: https://movies.disney.com/wall-e-gallery?. [Accessed 9 April 2020].

[26] J. James, V. Iyer, Y. Chukewad, S. Gollakota and S. B. Fuller, "Liftoff of a $190 \mathrm{mg}$ Laser-Powered Aerial Vehicle: The Lightest Wireless Robot to Fly," in 2018 IEEE International Conference on Robotics and Automation (ICRA), Brisbane, 2018.

[27] W. Hu, K. S. Ishii and A. T. Ohta, "Micro-assembly using optically controlled bubble microrobots in saline solution," in 2012 IEEE International Conference on Robotics and Automation, Saint Paul, 2012.

[28] A. Hsu, C. Cowan, W. Chu, B. McCoy, A. Wong-Foy, R. Pelrine, C. Velez, D. Arnold, J. Lake, J. Ballard and J. Randall, "Automated 2D micro-assembly using diamagnetically levitated milli-robots," in International Conference on Manipulation, Automation and Robotics at Small Scales (MARSS), Montreal, QC, Canada, 2017.

[29] R. Murthy, H. E. Stephanou and D. O. Popa, "AFAM: An Articulated Four Axes Microrobot for Nanoscale Applications," IEEE Transactions on Automation Science and Engineering, vol. 10, no. 2, pp. 276-284, 2013.

[30] S. Yang and N. Lu, "Gauge Factor and Stretchability of Silicon-on-Polymer Strain Gauges," Sensors, vol. 13, no. 7, p. 8577-8594, 2013. 
[31] S. Salmanipour and E. Diller, "Eight-Degrees-of-Freedom Remote Actuation of Small Magnetic Mechanisms," in IEEE International Conference on Robotics and Automation (ICRA), Brisbane, Australia, 2018.

[32] J. Zhang, M. Salehizadeh and E. Diller, "Parallel Pick and Place Using Two Independent Untethered Mobile Magnetic Microgrippers," in IEEE International Conference on Robotics and Automation (ICRA), Brisbane, Australia, 2018.

[33] J. N. Israelachvili, Intermolecular and Surface Forces, Waltham, MA: Elsevier, 2011.

[34] K. Y. Ma, P. Chirarattananon, S. B. Fuller and R. J. Wood, "Controlled Flight of a Biologically Inspired, Insect-Scale Robot," Science, vol. 340, no. 6132, pp. 603607, 2013.

[35] M. A. Graule, P. Chirarattananon, S. B. Fuller, N. T. Jafferis, K. Y. Ma, M. Spenko, R. Kornbluh and R. J. Wood, "Perching and takeoff of a robotic insect on overhangs using switchable electrostatic adhesion," Science, vol. 352, no. 6288, pp. 978-982, 2016.

[36] Y. Chen, H. Wang, E. F. Helbling, N. T. Jafferis, R. Zufferey, A. Ong, K. Ma, N. Gravish, P. Chirarattananon, M. Kovac and R. J. Wood, "A biologically inspired, flapping-wing, hybrid aerial-aquatic microrobot," Science Robotics, vol. 2, no. 11, 2017.

[37] N. T. Jafferis, E. F. Helbling, M. Karpelson and R. J. Wood, "Untethered flight of an insect-sized flapping-wing microscale aerial vehicle," Nature, vol. 570, pp. 491495, 2019.

[38] S. B. Fuller, Z. E. Teoh, P. Chirarattananon, N. O. Pérez-Arancibia, J. Greenberg and R. J. Wood, "Stabilizing air dampers for hovering aerial robotics: design, insectscale flight tests, and scaling," Autonomous Robots, vol. 41, no. 8, pp. 1555-1573, 2017.

[39] Y. M. Chukewad, A. T. Singh, J. M. James and S. B. Fuller, "A New Robot Fly Design That is Easier to Fabricate and Capable of Flight and Ground Locomotion," in 2018 IEEE/RSJ International Conference on Intelligent Robots and Systems (IROS), Madrid, 2018.

[40] S. B. Fuller, "Four Wings: An Insect-Sized Aerial Robot With Steering Ability and Payload Capacity for Autonomy," IEEE Robotics and Automation Letters, vol. 4, no. 2, pp. 570-577, 2019. 
[41] R. Murthy, A. Das and D. O. Popa, "ARRIpede: A stick-slip micro crawler/conveyor robot constructed via $21 / 2 \mathrm{D}$ MEMS assembly," in IEEE/RSJ International Conference on Intelligent Robots and Systems, Nice, France, 2008.

[42] R. Murthy, A. N. Das, D. O. Popa and H. E. Stephanou, "ARRIpede: An Assembled Die-Scale Microcrawler," Advanced Robotics, vol. 25, no. 8, pp. 965-990, 2011.

[43] R. Murthy, A. N. Das and D. O. Popa, "ARRIpede: An Assembled Micro Crawler," in 8th IEEE Conference on Nanotechnology, Arlington, TX, USA, 2008.

[44] O. Youssefi and E. Diller, "Contactless Robotic Micromanipulation in Air Using a Magneto-Acoustic System," IEEE Robotics and Automation Letters, vol. 4, no. 2, pp. 1580-1586, 2019.

[45] J. Zhang, M. Salehizadeh and E. Diller, "Parallel Pick and Place Using Two Independent Untethered Mobile Magnetic Microgrippers," in IEEE International Conference on Robotics and Automation (ICRA), Brisbane, QLD, Australia, 2018.

[46] P. Ryan and E. Diller, "Magnetic Actuation for Full Dexterity Microrobotic Control Using Rotating Permanent Magnets," IEEE Transactions on Robotics, vol. 33, no. 6, pp. 1398-1409, 2017.

[47] A. Hsu, A. Wong-Foy and R. Pelrine, "Ferrofluid Levitated Micro/Milli-Robots," in International Conference on Manipulation, Automation and Robotics at Small Scales (MARSS), Nagoya, Japan, 2018.

[48] S. Floyd, E. Diller and C. Pawashe, "Control methodologies for a heterogeneous group of untethered magnetic micro-robots," the International Journal of Robotics Research, vol. 30, no. 13, pp. 1553-1565, 2011.

[49] J. Li, X. Li, T. Luo, R. Wang, C. Liu, S. Chen, D. Li, J. Yue, S.-h. Cheng and D. Sun, "Development of a magnetic microrobot for carrying and delivering targeted cells," Science Robotics, vol. 3, no. 19, 2018.

[50] H. Xie, M. Sun, X. Fan, Z. Liu, W. Chen, L. Wang, L. Dong and Q. He, "Reconfigurable magnetic microrobot swarm: Multimode transformation, locomotion, and manipulation," Science Robotics, vol. 4, no. 28, 2019.

[51] P. Ryan and E. Diller, "Magnetic Actuation for Full Dexterity Microrobotic Control Using Rotating Permanent Magnets," IEEE Transactions on Robotics, vol. 33, no. 6, pp. 1398-1409, 2017. 
[52] W. Hu, K. S. Ishii and A. Ohta, "Micro-assembly using optically controlled bubbles," in 16th International Conference on Optical MEMS and Nanophotonics, Istanbul, Turkey, 2011.

[53] K. S. Ishii, W. Hu and A. T. Ohta, "Cooperative micromanipulation using optically controlled bubble microrobots," in IEEE International Conference on Robotics and Automation, Saint Paul, MN, USA, 2012.

[54] M. R. Pac and D. O. Popa, "3-DOF Untethered Microrobot Powered by a Single Laser Beam Based on Differential Thermal Dynamics," in 2011 IEEE International Conference on Robotics and Automation, Shanghai, China, 9-13 May 2011.

[55] A. T. Baisch and R. J. Wood, "Design and Fabrication of the Harvard Ambulatory Micro-Robot," Springer Tracts in Advanced Robotics, vol. 70, pp. 715-730, 2011.

[56] R. Sahai, S. Avadhanula, R. Groff, E. Steltz, R. Wood and R. S. Fearing, "Towards a $3 \mathrm{~g}$ crawling robot through the integration of microrobot technologies," in Proceedings 2006 IEEE International Conference on Robotics and Automation, Orlando, FL, USA, 2006.

[57] A. G. Dharmawan, H. H. Hariri, S. Foong, G. S. Soh and K. L. Wood, "Steerable Miniature Legged Robot Driven by a Single Piezoelectric Steerable Miniature Legged Robot Driven by a Single Piezoelectric," in 2017 IEEE International Conference on Robotics and Automation (ICRA), Singapore, May 29 - June 3, 2017.

[58] S. A. Rios, A. J. Fleming and Y. K. Yong, "Design and Characterization of a Miniature Monolithic Piezoelectric Hexapod Robot," in 2016 IEEE International Conference on Advanced Intelligent Mechatronics (AIM), Banff, Alberta, Canada, July $12-15,2016$.

[59] E. Y. Erdem, Y.-M. Chen, M. Mohebbi, J. W. Suh, G. T. A. Kovacs, R. B. Darling and K. F. Böhringer, "Thermally Actuated Omnidirectional Walking Microrobot," Journal of Microelectromechanical Systems, vol. 19, no. 3, pp. 433-442, June 2010.

[60] B. R. Donald, C. G. Levey, C. D. McGray, I. Paprotny and D. Rus, "An Untethered, Electrostatic, Globally Controllable MEMS Micro-Robot," Journal of Microelectromechanical Systems, vol. 15, no. 1, pp. 1 - 15, February 2006.

[61] P. Ryan and E. Diller, "Five-Degree-of-Freedom Magnetic Control of MicroRobots Using Rotating Permanent Magnets," in 2016 IEEE International Conference on Robotics and Automation (ICRA), Stockholm, Sweden, May 16-21, 2016. 
[62] S. Ward, V. Foroutan, R. Majumdar, O. Mahdavipour, S. A. Hussain and I. Paprotny, "Towards Microscale Flight: Fabrication, Stability Analysis, and Initial Flight Experiments for $300 \mu \mathrm{m} \times 300 \mu \mathrm{m} \times 1.5 \mu \mathrm{m}$ Sized Untethered MEMS Microfliers," IEEE Transactions on Nanobioscience, vol. 14, no. 3, pp. 323-331, April, 2015.

[63] F. R. Szabo and P. E. Kladitis, "Design, Modeling and Testing of Polysilicon Optothermal Actuators for Power Scavenging Wireless Microrobots," in 2004 International Conference on MEMS, NANO and Smart Systems (ICMENS'04), Banff, AB, Canada, Canada, 25-27 Aug. 2004.

[64] M. Tecpoyotl-Torres, J. Varona, A. A. Hamoui, J. Escobedo-Alatorre and J. Sanchez-Mondragón, "Polysilicon thermal micro-actuators for heat scavenging and power conversion," in Proceedings Volume 7043, High and Low Concentration for Solar Electric Applications III, San Diego, California, United States, 2008.

[65] C. Pawashe, S. Floyd and M. Sitti, "Modeling and Experimental Characterization of an Untethered Magnetic Micro-Robot," The International Journal of Robotics Research, vol. 28, no. 8, pp. 1077-1094, 2008.

[66] D. R. Frutiger, K. Vollmers, B. E. Kratochvil and B. J. Nelson, "Small, Fast, and Under Control: Wireless Resonant Magnetic Micro-agents," The International Journal of Robotics Research, vol. 29, no. 5, pp. 613-636, 2010.

[67] W. Hu, G. Z. Lum, M. Mastrangeli and M. Sitti, "Small-scale soft-bodied robot with multimodal locomotion," Nature, vol. 554, pp. 81-85, 2018.

[68] M. R. Pac and D. O. Popa, "Laser-Powered Sub-mm Untethered Microrobots," in Proceedings of the ASME 2010 Design and Engineering Technical Conferences \& Computers and Information in Engineering Conference, Montreal, Quebec, Canada, August 15-18, 2010.

[69] R. Zhang, A. Sherehiy, Z. Yang, D. Wei, C. K. Harnett and D. O. Popa, "ChevBot - An Untethered Microrobot Powered by Laser for Microfactory Applications," in 2019 International Conference on Robotics and Automation, Montreal, Canada, 2019.

[70] R. Zhang, A. Sherehiy, D. Wei, Z. Yang, M. N. Saadatzi and D. O. Popa, "Tracking Experiments with ChevBot: A Laser-Actuated Stick-Slip Microrobot," in International Conference on Manipulation Automation and Robotics at Small Scales(MARSS), Helsinki, Finland, 2019. 
[71] K. Tsui and A. Geisberger, "Sockets for microassembly". United States Patent US7025619B2, 1322004.

[72] A. Geisberger, G. Skidmore and K. Tsui, "Microconnectors and non-powered microassembly therewith". United States Patent EP1564183A2, 1322004.

[73] R. Zhang, D. Wei and D. O. Popa, "Design, Analysis and Fabrication of sAFAM, a 4 DoF Assembled Microrobot," in International Conference on Manipulation, Automation and Robotics at Small Scales (MARSS), Nagoya, Japan, 2018.

[74] R. Zhang, A. Sherehiy, D. Wei and D. O. Popa, "Design and characterization of solid articulated four axes microrobot for microfactory applications," Journal of Micro-Bio Robotics, pp. 1-13, 2019.

[75] P. H. Tsang, G. Li, Y. V. Brun, L. B. Freund and J. X. Tang, "Adhesion of single bacterial cells in the micronewton range," PNAS, vol. 103, no. 15, pp. 5764-5768, 2006.

[76] S. Achanta, D. Drees and J.-P. Celis, "Investigation of friction on hard homogeneous coatings during reciprocating tests at micro-Newton normal forces," Wear, vol. 263, no. 7-12, pp. 1390-1396, 2007.

[77] J. D. Whittaker, M. D. Ethan, D. M. Tanenbaum, P. L. McEuen and R. C. Davis, "Measurement of the Adhesion Force between Carbon Nanotubes and a Silicon Dioxide Substrate," Nano Letters, vol. 6, no. 5, pp. 953-957, 2006.

[78] M. Tanaka, "Development of desktop machining microfactory," RIKEN Review: Focused on Advances on Micro-mechanical Fabrication Techniques, no. 34, pp. 4649, 2001.

[79] Z. Zhakypov, T. Uzunovic, A. O. Nergiz, E. A. Baran, E. Golubovic and A. Sabanovic, "Desktop microfactory for high precision assembly and machining," in IEEE 23rd International Symposium on Industrial Electronics (ISIE), Istanbul, Turkey, 2014.

[80] C. Diederichs, M. Mikczinski and T. Tiemerding, "A Flexible and Compact High Precision Micro-Factory for Low Volume Production and Lab-Automation," in 41st International Symposium on Robotics, Munich, Germany, 2014.

[81] Z. Zhakypov, T. Uzunovic, A. O. Nergiz, E. A. Baran, E. Golubovic and A. Sabanovic, "Modular and reconfigurable desktop microfactory for high precision manufacturing," The International Journal of Advanced Manufacturing Technology, vol. 90, no. 9-12, pp. 3749-3759, 2017. 
[82] R. Murthy and D. O. Popa, "Millimeter-scale microrobots for wafer-level factories," in IEEE International Conference on Robotics and Automation, Anchorage, AK, USA, 2010.

[83] R. Pelrine, A. Wong-Foy, A. Hsu and B. McCoy, "Self-assembly of milli-scale robotic manipulators: A path to highly adaptive, robust automation systems," in International Conference on Manipulation, Automation and Robotics at Small Scales (MARSS), Paris, France, 2016.

[84] J. Zhang, O. Onaizah, K. Middleton, L. You and E. Diller, "Mobile Magnetic Microgripper for Autonomous Pick-and-Place," IEEE Robotics and Automation Letters, vol. 2, no. 2, pp. 835-840, 2017.

[85] R. Murthy, A. N. Das and D. O. Popa, "Nonholonomic Control for an Assembled Microcrawler," IFAC Proceedings Volumes, vol. 42, no. 16, pp. 627-632, 2009.

[86] R. Pelrine, A. Hsu, C. Cowan and A. Wong-Foy, "Multi-Agent Systems Using Diamagnetic Micro Manipulation - From Floating Swarms to Mobile Sensors," in International Conference on Manipulation, Automation and Robotics at Small Scales (MARSS), Montreal, QC, Canada, 2017.

[87] H. Woern, M. Szymanski and J. Seyfried, "The I-SWARM project," in The 15th IEEE International Symposium on Robot and Human Interactive Communication, Hatfield, UK, 2006.

[88] S. S. Shroff and M. P. de Boer, "Constant Velocity High Force Microactuator for Stick-Slip Testing of Micromachined Interfaces," Journal of Microelectromechanical Systems, vol. 24, no. 6, pp. 1868-1877, 2015.

[89] S. Fahlbusch, S. Fatikow, J. Seyfried and A. Buerkle, "Flexible microrobotic system MINIMAN: design, actuation principle and control," in IEEE/ASME International Conference on Advanced Intelligent Mechatronics, Atlanta, GA, USA, 1999.

[90] Y. Okazaki, N. Mishima and K. Ashida, "Microfactory-Concept, History, and Developments," Journal of Manufacturing Science and Engineering, vol. 126, no. 4, pp. 837-844, 2005.

[91] C. Clevy, A. Hubert, J. Agnus and N. Chaillet, "A micromanipulation cell including a tool changer," Journal of micromechanics and microengineering, no. 15, pp. 292$301,2005$.

[92] R. H. Heikkila, I. T. Karjalainen, J. J. Uusitalo, A. S. Vuola and R. O. Tuokko, "Possibilities of a Microfactory in the Assembly of Small Parts and Products - First 
Results of the M4-project," in IEEE International Symposium on Assembly and Manufacturing, Ann Arbor, MI, USA, 2007.

[93] I. Verettas, R. Clavel and A. Codourey, "Microfactory :Desktop Cleanrooms for the Production of Microsystems," in IEEE International Symposium on Assembly and Task Planning, Besancon, France, 2003.

[94] I. Verettas, R. Clavel and A. Codourey, "PocketFactory: a modular and miniature assembly chain including a clean environment," in International Workshop on MicroFactories (IWMF), Besançon, France, 2006.

[95] S.-H. Jang, Y.-M. Jung, H.-Y. Hwang, Y.-H. Choi and J.-K. Park, "Development of a Reconfigurable Micro Machine Tool for Microfactory," in International Conference on Smart Manufacturing Application, Gyeonggi-do, South Korea, 2008.

[96] N. Siltala, T. Prusi, A. Vuola, R. Heikkilä and R. Tuokko, "Modular microfactory system for gas sensor assembly," in IEEE International Symposium on Assembly and Manufacturing (ISAM), Tampere, Finland, 2011.

[97] N. Mishima, S. Kondo and K. Masui, "A Study on Efficiency Analysis of Micro Manufacturing Systems," in IEEE International Conference on Mechatronics and Automation, Harbin, China, 2007.

[98] N. Kawahara, T. Hirano, Y. Ishikawa, T. Kitahara, N. Ooyama and T. Ataka, "Microfactories; new applications of micromachine technology to the manufacture of small products," Microsystem Technologies, vol. 3, no. 2, pp. 37-41, 1997.

[99] E. Kussul, T. Baidyk, L. Ruiz-Huerta, A. Caballero-Ruiz, G. Velasco and L. Kasatkina, "Development of micromachine tool prototypes for microfactories," Journal of Micromechanics and Microengineering, vol. 12, pp. 795-812, 2002.

[100] Z. Zhakypov, E. Golubovic and A. Sabanovic, "Galvanometric optical laser beam steering system for microfactory application," in IECON 2013 - 39th Annual Conference of the IEEE Industrial Electronics Society, Vienna, Austria, 2013.

[101] L. Lévesque, "Law of cooling, heat conduction and Stefan-Boltzmann radiation laws fitted to experimental data for bones irradiated by $\mathrm{CO} 2$ laser," Biomedical Optics Express, vol. 5, no. 3, pp. 701-712, 2014.

[102] F. P. Incropera, Introduction to Heat Transfer 6th Edition, John Wiley \& Sons, Inc., 2011. 
[103] M. A. Green, "Self-consistent optical parameters of intrinsic silicon at $300 \mathrm{~K}$ including temperature coefficients," Solar Energy Materials and Solar Cells, vol. 92, no. 11, pp. 1305-1310, 2008.

[104] L. Que, J.-S. Park and Y. B. Gianchandani, "Bent-beam electrothermal actuatorsPart I: Single beam and cascaded devices," Journal of Microelectromechanical Systems, vol. 10, no. 2, pp. 247 - 254, Jun 2001.

[105] Z. Yang and D. O. Popa, "A General Kinematic Modeling Framework for a 3D Compliant Micromechanism," in International Conference on Manipulation, Automation and Robotics at Small Scales, Nagoya, Japan, 2018.

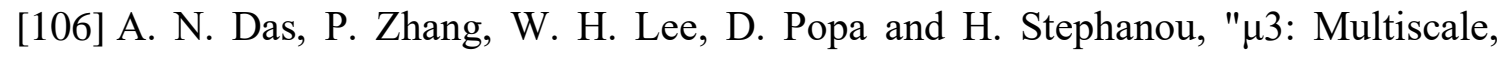
Deterministic Micro-Nano Assembly System for Construction of On-Wafer Microrobots," in Proceedings 2007 IEEE International Conference on Robotics and Automation, Roma, Italy, 2007.

[107] J.-S. Park, L. L. Chu, A. D. Oliver and Y. B. Gianchandani, "Bent-beam electrothermal actuators-Part II: Linear and rotary microengines," Journal of Microelectromechanical Systems, vol. 10, no. 2, pp. 255-262, 2001.

[108] R. Cragun and L. L. Howell, "Linear Thermomechanical Microactuators," ASME MEMS, vol. 1, pp. 181-188, 1999.

[109] J. M. Maloney, D. S. Schreiber and D. L. DeVoe, "Large-force electrothermal linear micromotors," Journal of Micromechanics and Microengineering, vol. 14, no. 2, pp. 226-234, 2004.

[110] M. J. Madou, Fundamentals of Microfabrication The Science of Miniaturization, CRC Press, 2002.

[111] M. A. Hopcroft, W. D. Nix and T. W. Kenny, "What is the Young's Modulus of Silicon?," Journal of Microelectromechanical Systems, vol. 19, no. 2, pp. 229-238, 2010.

[112] J. M. Breguet and R. Clavel, "Stick and slip actuators: design, control, performances and applications," in International Symposium on Micromechatronics and Human Science, Nagoya, Japan, 1998.

[113] J. F. Klotz, D. Wei, Z. Yang, R. Zhang, A. Sherehiy, M. Saadatzi and D. O. Popa, "Concept Validation for a Novel Stick-and-Slip, Light-Powered, Mobile MicroCrawler," in International Conference on Manipulation Automation and Robotics at Small Scales, Helsinki, Finland, 2019. 
[114] Z. Yang, M. N. Saadatzi, R. Zhang, A. Sherehiy, D. Wei, K. C. Harnett and D. O. Popa, "Multiphysics Dynamic Model Validation Methodology for Laser-Driven Microrobots," in 2019 IEEE 15th International Conference on Automation Science and Engineering, vancouver, 2019.

[115] SPECTROLAB INC., "Concentrator Cells," SPECTROLAB INC., 2222020. [Online]. Available: http://www.spectrolab.com/photovoltaics.html\#concentrator. [Accessed 222 2020].

[116] Laird Connectivity, "BL652 Series - Bluetooth v5 + NFC," 295 2019. [Online]. Available: https://connectivity-staging.s3.us-east-2.amazonaws.com/2019-05/CSDS-BL652\%20v2_9.pdf. [Accessed 102 2020].

[117] Edmund Optics®, "Gaussian Beam Propagation," Edmund Optics, 1832020. [Online]. Available: https://www.edmundoptics.com/knowledgecenter/application-notes/lasers/gaussian-beam-propagation/. [Accessed 183 2020].

[118] PVeducation, "Optical Properties of Silicon," 163 2020. [Online]. Available: https://www.pveducation.org/pvcdrom/materials/optical-properties-ofsilicon\#footnote1_7c0q6s2. [Accessed 163 2020].

[119] R. Zhang, Z. Yang, D. Wei and P. Dan, "Design and fabrication of an articulated four axes microrobot arm," in Proceedings Volume 10216, Smart Biomedical and Physiological Sensor Technology XIV, Anaheim, California, United States, 2017.

[120] J. Baptist, R. Zhang, D. Wei, M. N. Saadatzi and D. O. Popa, "Fabrication of strain gauge based sensors for tactile skins," in Proceedings Volume 10216, Smart Biomedical and Physiological Sensor Technology XIV, Anaheim, California, United States, 2017. 


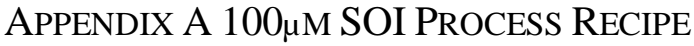

1. RCA clean the sample wafer.

2. Dip the sample in BOE solution for 1 minute.

3. Load the sample wafer into sputter Lesker PVD-75, vacuum the chamber down below $5 \times 10^{-5}$ Torr.

4. Make sure the sample chuck is rotating by check "Platen Motion", set target Argon flow pressure to $5 \mathrm{mT}$ Torr, and wait for the pressure reading become stable.

5. Make sure the DC power is set to $300 \mathrm{~W}$, on power supply 3.

6. Pre-sputter Chromium for at least 60 seconds, without open the sample shutter, to allow oxide layer to be removed.

7. Sputter Chromium for 2 minutes then gold for 4 minutes. Let the gold target rest 1 minute after the first 2 minutes.

8. Spin photoresist Shipley 1813 with recipe \#2 on a spinner: 1 second for 500 RPM to spread then 30 seconds for 3000 RPM. Soft bake 2 minutes.

9. Align the "Metal" mask with the wafer under mask aligner SÜSS MA6/BA6, make sure the design pattern roughly projects to the center of the wafer.

10. Expose 11 seconds by hard contact (over exposure to ensure fully development and minimize metal residue later).

11. Develop 70+ seconds under MF-319 from MicroChem.

12. Inspect the sample under microscope, make sure all features are intact.

13. Hard bake 5 minutes on hot plate, under $115^{\circ} \mathrm{C}$ 
14. Etch gold and chromium with corresponding etchant. A little bit over etch is preferred. Do not dilute gold etchant. 1 minute 15 seconds gives both full over etch. Inspect alignment marks make sure it is intact.

15. Remove photoresist and use the Trion etcher (Minilock-Phantom III ICP/RIE) to perform an oxygen plasma clean for 20 minutes to remove any residue and achieve maximum cleanness.

16. Apply HMDS (optional, only if later photolithography step fails).

17. Spin photoresist SPR220-3.0 on a spinner, 2 seconds for 500 RPM then 30 seconds for 3000 RPM.

18. Soft bake: 3 minutes bake on $45^{\circ} \mathrm{C}$ hot plate, 3 minutes on $90^{\circ} \mathrm{C}, 5$ minutes on $115^{\circ} \mathrm{C}, 3$ minutes $90^{\circ} \mathrm{C}$ and 3 minutes $45^{\circ} \mathrm{C}$.

19. Align the "DRIE" mask with the metal layer under mask aligner and expose 16.5 seconds by hard contact.

20. Develop 60 seconds in MF-319 developer.

21. Inspect photoresist features under microscope to check photolithography quality and cover any exposed metal specks with the same type of photoresist by a Qtip.

22. Hard bake: 3 minutes bake on $45^{\circ} \mathrm{C}$ hot plate, 3 minutes on $90^{\circ} \mathrm{C}, 10$ minutes on $115^{\circ} \mathrm{C}, 3$ minutes $90^{\circ} \mathrm{C}$ and 3 minutes $45^{\circ} \mathrm{C}$.

23. Deep reactive ion etching (DRIE) on the STS Multiplex ASE Advanced Silicon Etcher. Character the etch rate base on 10 minutes etch and finish the rest. Expected etching time is between 20 to 30 minutes. CONSULT CLEANROOM STAFF FOR BEST RESULT. 
24. DO NOT USE OXYGEN PLASMA CLEAN OF THE DRIE MACHINE SINCE EXPOSED METAL WILL CONTAMINATE THE CHAMBER.

25. Remove photoresist mask with NMP, and preserve the wafer with Shipley 1813 photoresist, under 500 RPM for 30 seconds, then hard bake 5 minutes. This step protects the patterned wafer surface from contamination due to dicing.

26. Dice.

27. Rinse each die with acetone, isopropyl alcohol and de-ionized water to remove photoresist.

28. Oxygen plasma clean with Trion etcher to further clean the surface.

29. Release the die with vapor HF tool.

30. Prob the sample to guarantee the etching is done.

31. Preserve the rest of the samples. 
1. RCA clean the sample wafer.

2. Dip the sample in BOE solution for 1 minute.

3. Apply HMDS (optional, only if later photolithography step fails).

4. Spin photoresist SPR220-3.0 on a spinner, 2 seconds for 500 RPM then 30 seconds for 3000 RPM.

5. Soft bake: 3 minutes bake on $45^{\circ} \mathrm{C}$ hot plate, 3 minutes on $90^{\circ} \mathrm{C}, 5$ minutes on $115^{\circ} \mathrm{C}, 3$ minutes $90^{\circ} \mathrm{C}$ and 3 minutes $45^{\circ} \mathrm{C}$.

6. Align the "DRIE" mask with the sample wafer under the mask aligner, and expose 16.5 seconds by hard contact.

7. Develop 60 seconds in MF-319 developer.

8. Inspect photoresist features under microscope to check photolithography quality.

9. Hard bake: 3 minutes bake on $45^{\circ} \mathrm{C}$ hot plate, 3 minutes on $90^{\circ} \mathrm{C}, 10$ minutes on $115^{\circ} \mathrm{C}, 3$ minutes $90^{\circ} \mathrm{C}$ and 3 minutes $45^{\circ} \mathrm{C}$.

10. Deep reactive ion etching (DRIE) on the STS Multiplex ASE Advanced Silicon Etcher. Character the etch rate base on 3 minutes etch and finish the rest. Expected etching time is between 10 to 15 minutes. CONSULT CLEANROOM STAFF FOR BEST RESULT.

11. Use oxygen plasma clean recipe of the DRIE machine to remove the photoresist. 
12. Preserve the wafer with Shipley 1813 photoresist, under 500 RPM for 30 seconds, then hard bake 5 minutes. This step protects the patterned wafer surface from contamination due to dicing.

13. Dice.

14. Rinse each die with acetone, isopropyl alcohol and de-ionized water to remove photoresist.

15. Oxygen plasma clean with Trion etcher to further clean the surface.

16. Release the die with vapor HF tool.

17. Prob the sample to guarantee the etching is done.

18. Preserve the rest of the samples. 


\section{APPENDIX C ChEvBot's TRACKING EXPERIMENT DESIGN}

In order to verify and demonstrate ChevBot's locomotion, an automated power delivery and tracking system was implemented, and its technical details are presented here.

\section{C.1 Fix the Stage, or the Laser?}

Generally speaking, such experiment can be conducted in two ways. Either fixing operating surface and guide laser to track the ChevBot or fixing the laser onto ChevBot's body and track its motion by compensating the operating surface underneath, as illustrated in Figure C-1 (a) and (b) respectively. The first approach is the most natural thinking and the desired way of using ChevBot in a microfactory setup. However, it is more difficult to implement than the second approach.
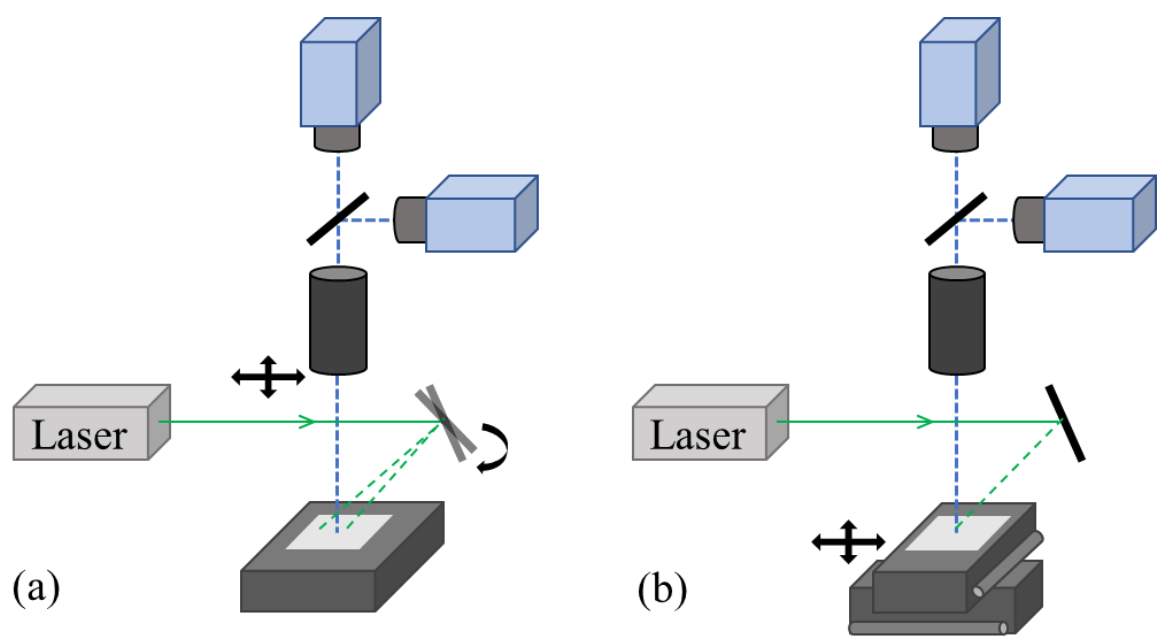

Figure C-1 Schematic of two approaches: (a) fix sample but adjust laser and camera. (b) compensate motion by stages.

In the experiment design, there four elements need to be considered: a ChevBot on an operating surface (the sample), stages that carries the sample, the laser delivery system, 
and the camera system. Of the four, the camera and laser systems are the most bulky and sophisticated parts, they all require accurate lens system to be precisely constructed so that the laser spot and images are in focus, and not impeding each other. For example, before the ChevBot's image entering camera, the laser spot needs to be filtered out so the ChevBot is visible since the laser spot is extremely bright. If we implement the first approach, a mirror galvanometer installed within the laser delivery system is needed to redirect/scan the laser beam onto the ChevBot body in the event of a generated locomotion. Meanwhile, the positions of the cameras may also need to be adjusted on-the-fly so that microrobot is in the field-of-view. So, we wish not to introduce motion into those two parts in the prototyping stage of the design.

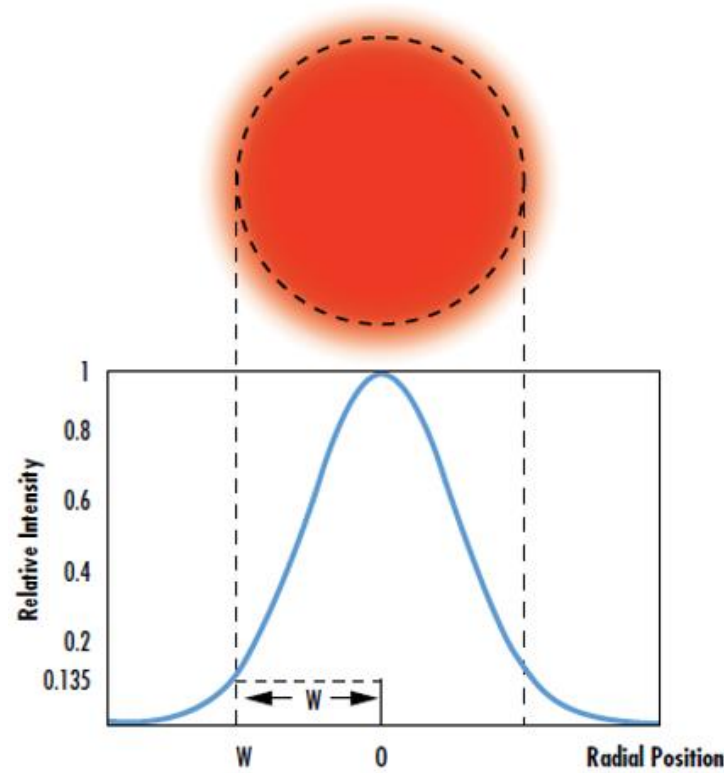

Figure C-2 Gaussian beam intensity illustration [117].

Regardless of the either approaches, visual servoing is another important piece of the puzzle. The irradiation power of the laser spot follows Gaussian distribution, as shown in Figure C-2. In order to couple the on-off cycle from laser onto the heating-cooling cycle of the thermal actuators, it requires the thermal actuators within the effective range of the 
Gaussian beam, as illustrated in Figure C-3. Which imposes the position feedback be provided in a timely manner. Position of the ChevBot on the operating surface guides the laser beam or the motorized stages so that energy can be deposited to the microrobot constantly. In order to successfully track the ChevBot, such feedback loop should be done as fast as possible to avoid the microrobot moves out of the range of the laser spot. In the event of the two cycles out-of-sync, the ChevBot will be out of the effective zone of the laser beam, hence its motion seizes.

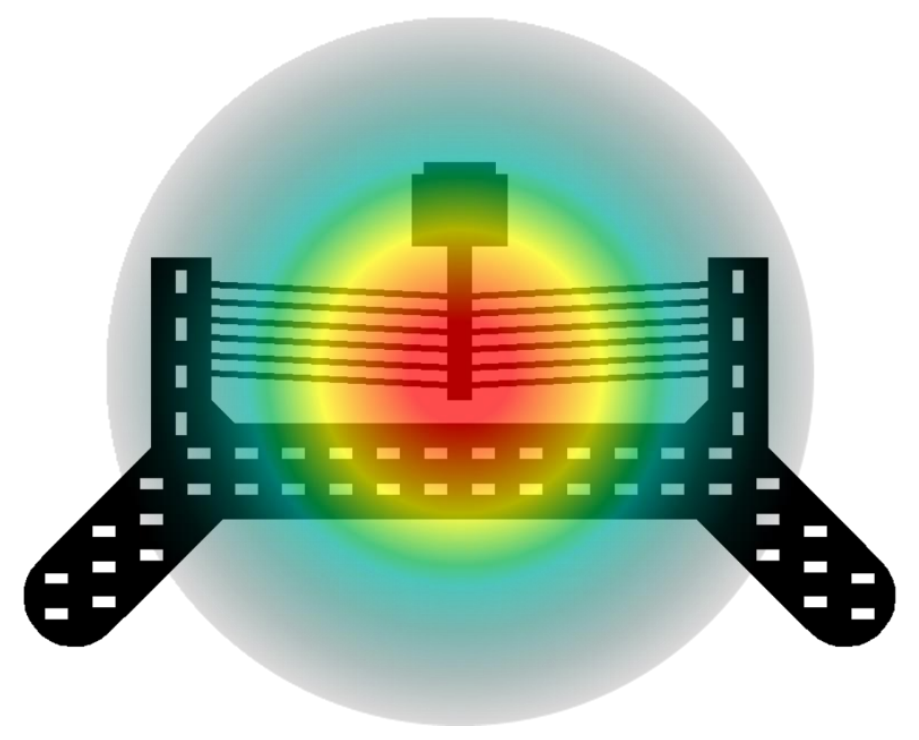

Figure C-3 Illustration of an ideal Gaussian beam on ChevBot's thermal actuators.

On the contrary, we can easily find reliable, off-the-shelf stages with high resolution to compensate ChevBot's motion. Meanwhile, LabVIEW application programming interface (API) in most name-brand stages are provided, so that integrating the full feedback loop controller on a PC is feasible. Besides, we already own a smart camera from National Instruments ${ }^{\circledR}$ to fulfill the closed-loop-control. As a result, the second approach with motorized stage was implemented. 


\section{C.2 Experiment Hardware Configuration}

The technical details of the experiment setup are discussed here, in the sequence of ChevBot and its operating surface, the laser delivery system, the motorized stages, and the camera system.

To prepare the experiment subject, an assembled ChevBot should sit on a piece of Silicon (arena) with the dimple and the two feet touching the surface at the same time. Here, special notice must be given to the ChevBot's placement, due to its very light body weight and the dimple has a flat surface, thus it easily sits on its dimple. If such situation happens, the ChevBot do not move under stimulus of laser beam and can be recognize by microscopy. Since the lighting was co-axial, a very reflective surface, such as ChevBot's body, is very bright if it is perpendicular to the light otherwise it is dark. A micro-manipulator with a clean probe can be used to touch the body to help it sit. The operating surface is a piece of Silicon, minimum dust contamination is preferred.

To verify the laser introduced gaiting cycle of the ChevBot, a laser module must have flexible repetition rate, preferable wavelength, adequate power output, and programming capability. According to preliminary study [118], Silicon's light absorption coefficient and wavelength have a negative correlation. To fulfill above requirements, we selected HE532200 laser module from Spectra-Physics®, the wavelength of such model is $532 \mathrm{~nm}$. A mechanical shutter (UniBlitz ${ }^{\circledR}$ T132) was placed in front of the laser outlet to manually control the on-off of light beam emission. The laser module is controlled on a PC, with tunable repetition rate, supply current, and amplitude. All three factor together decides the power output reading. 
Two motorized stages, placed perpendicular to each other, were used in the tracking experiments. Specifically, they are 423 linear translation stages from Newport ${ }^{\circledR}$, driven by CONEX-TRA25CC linear actuators/controller combo. The two motorized stages sit on another pair of manual X-Y stages with longer travel, so they have coarse position adjustment. To interfacing the CONEX stage controllers, a PC installed Microsoft ${ }^{\circledR}$ Windows 7 must be used since the CONEX controllers do not support any higher version of Windows. As a side note, Intel removed Windows 7 support after Skylake chipset. A dedicated PC running 32-bit LabVIEW 2015 was used to interfacing the stage controllers.

Position feedback of the ChevBot is provided by a smart camera (NI-1772C) from National Instruments®. This is a unique product that a CCD into an embedded desktop computer, so the image processing is done within the smart camera and sends data to the controller PC. In order to configure and receive data from the smart camera, it has to be within the same local network with the controller PC. The easiest way is to hook up the PC and the camera into a router.

\section{C.3 Controller Program Design}

Two individual programs were implemented on a dedicated controller PC: inverse image Jacobian matrix calculation and ChevBot tracking. They were written with LabVIEW 2015, 32-bit version and briefly discussed in this section.

\section{C.3.1 Inverse Image Jacobian Calculation}

An inverse image Jacobian matrix relates pixel location change in the camera space with the stage location change. Then the calculated matrix is manually inputted into the tracking program. The smart camera is a necessary component for both programs thus configured first with NI Vision Builder 2015 32-bit version, which is a separate software installed with 
the controller PC. Three steps were implemented within the smart camera: image threshold processing to help locate the target, image template matching locate where the ChevBot is, and extract location information. With all steps defined and saved in the smart camera as an "inspection", then they can be called by LabVIEW APIs.

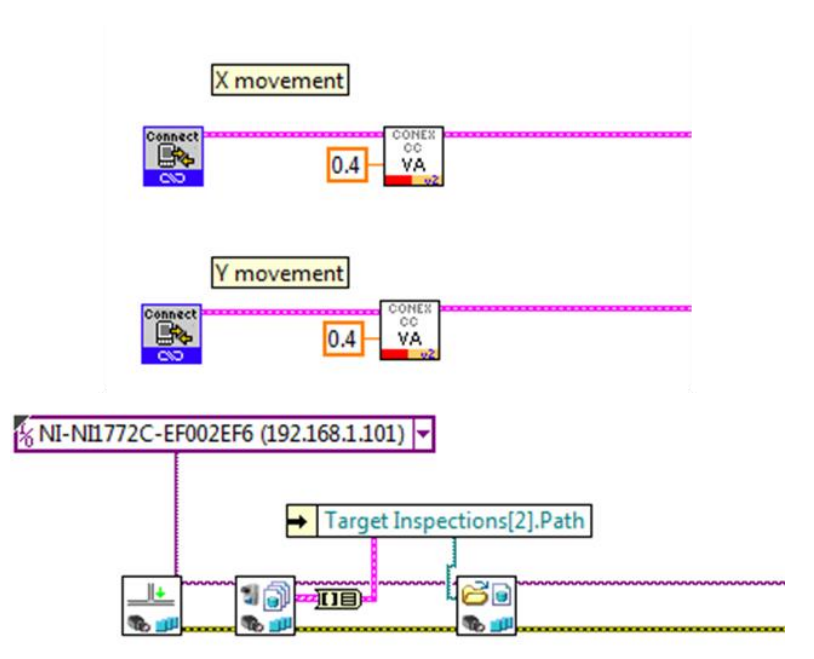

Figure C-4 Stage and smart camera initialization.

In the inverse Jacobian calculation program, the two stages were connected, and their motion speed was set. Then the smart camera is connected by the "Open Connection.vi". If the Ethernet connection is properly set, the smart camera should show up at the "session in" port. Figure C-4 shows the initialization code. The controller PC commands the execution of the inspection script, the smart camera sends $\mathrm{X}, \mathrm{Y}, \theta$ back to PC after it processes the position information. As shown in Figure C-5.

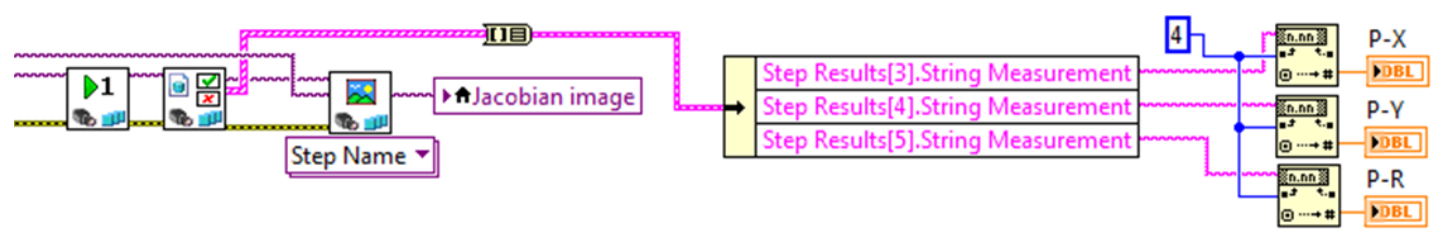

Figure C-5 Smart camera pixel position acquisition. 
To calculate the inverse image Jacobian matrix, we ran the stages to multiple points (larger than 5) to collect their pixel location, meanwhile, the stage position readings were also recorded. By apply algorithm in Figure 3-7, the matrix can be derived. Figure C-6 shows the LabVIEW code used to generate the random stages positions.

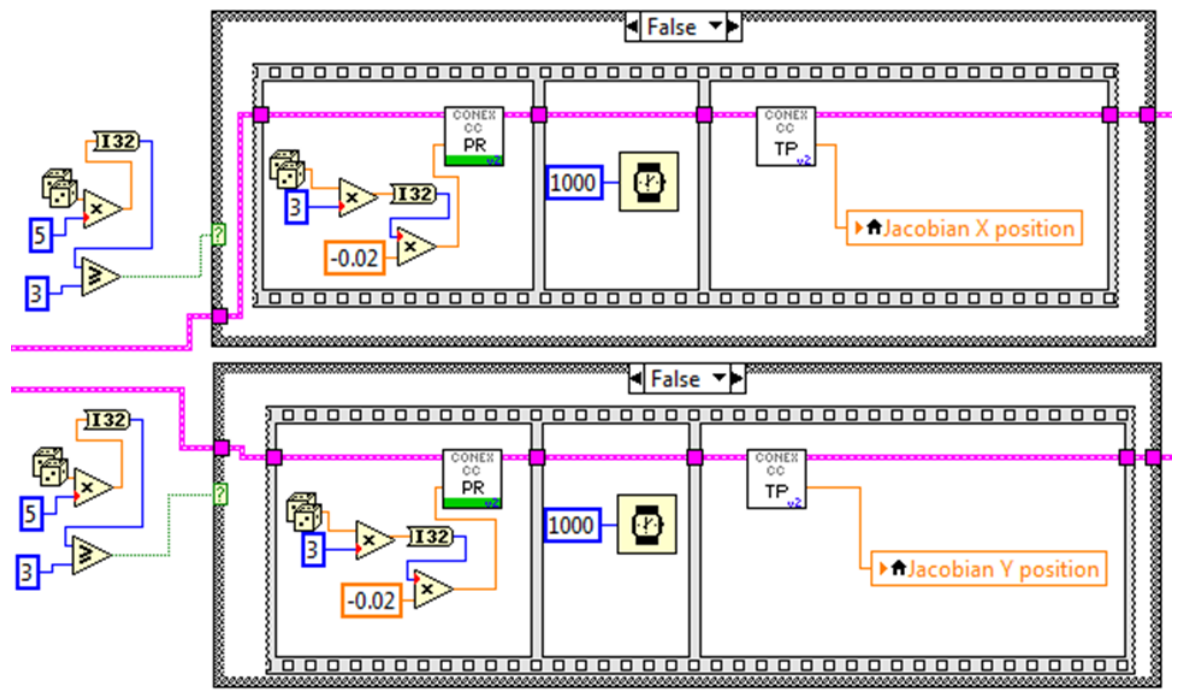

Figure C-6 Stage random step generation.

\section{C.3.2 ChevBot's Tracking Program}

Tracking program reused the initialization code to start. In the tracking task, we wish to keep the ChevBot at the center of the field of view. Represented by pixel number with a $640 \times 480$ image sensor, the center pixel is 320 by 240 , this is the ChevBot's target position. With that in mind, we can calculate the difference between ChevBot's current position and the desired position, which generates an error term e. Then we can convert the error term from pixel space to real distance with the help of the inverse image Jacobian matrix. If the distance error exceeds a certain empirical threshold, it is used to drive the stage with a fixed gain. Above controller is equivalent to a proportional controller. 


\section{APPENDIX D SOLARPEDE'S HARDWARE DESIGN}

The technical details of SolarPede design is presented here. The SolarPede microrobot is a heterogeneous microrobot consists of two major subsystems, the MEMS die and the electronics backpack. The technical aspect of circuity design and construction of the electronics backpack are present here.

The electronic circuit design was design with a free PCB design package KiCAD, version 5.1.5 stable release, on a Microsoft Windows machine. The electronics backpack has two PCBs: the top (power) board carries the solar cells and voltage boosting circuits, and the bottom (control) board carries the Bluetooth MCU, MOSFET switch array and the MEMS base.

\section{D.1 Power Board Design and Construction}

The circuit schematic of the power board is shown in Figure D-1, and the PCB is a 2layers board.

In order to connect the four solar cells in serial configuration and keep the board flat and tidy, the cells were "stitched" through the board. The two terminal bars on either side of the solar cells are their cathodes and the whole bottom side is the anode. To mount the solar cell, first we glue the solar cells to the PCB, then solder a thin wire to connect one terminal bar to the adjacent bonding pad. Second, this process was repeated to solder the anode to the front side of the board by the through hole. As indicated by Figure D-2 and 
the yellow wires in Figure D-3. The rest of the components are conventional surface-mount parts and can be assembled by hand.

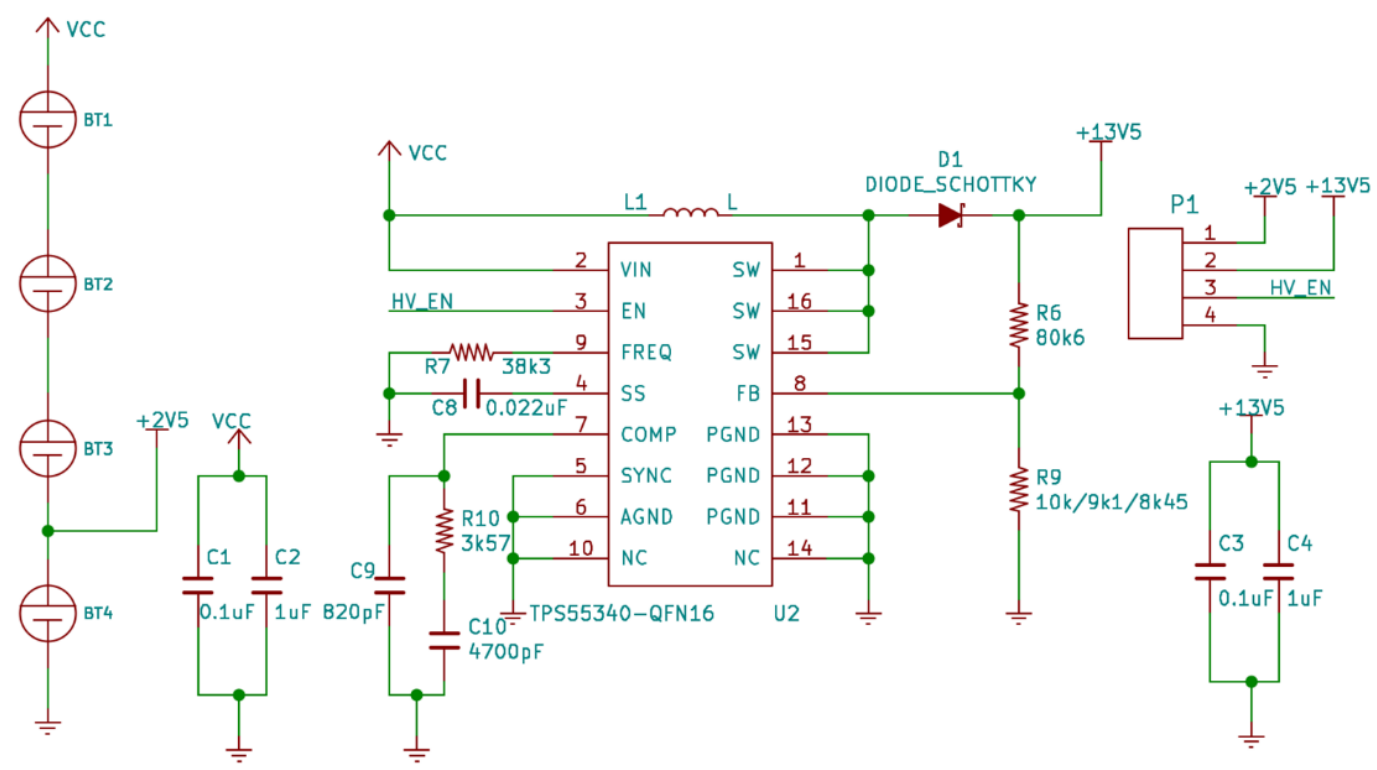

Figure D-1 Circuit schematic of the top board.

The front and bottom side of the top PCB are shown in Figure D-2 and Figure D-3, respectively:

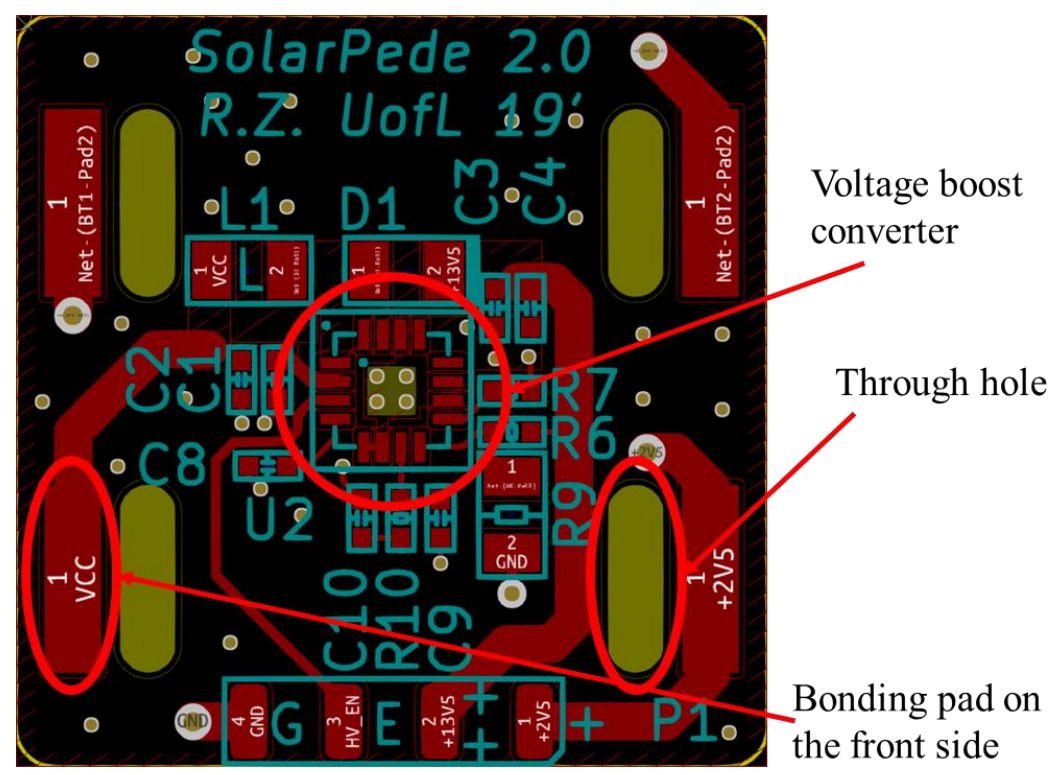

Figure D-2 Front side of the top PCB. 


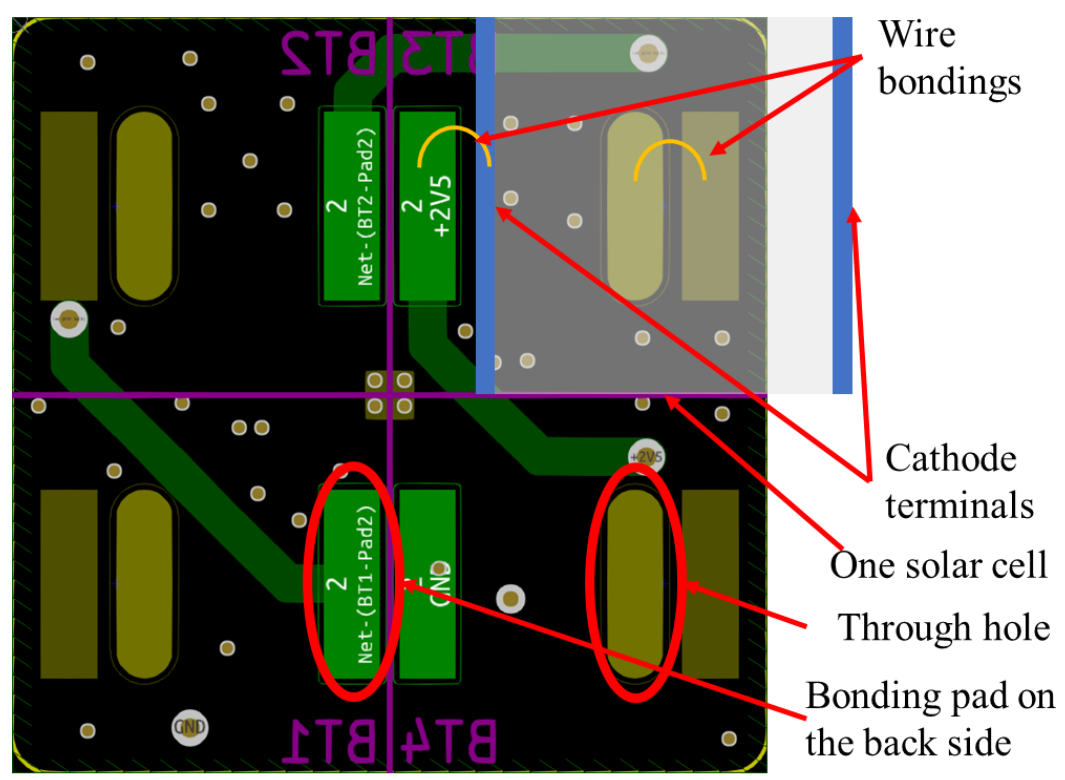

Figure D-3 Bottom side of the top PCB. Each quadrant is a solar cell mounting site. One site shows how the solar cells are mounted to the bottom side of the board, the cell is represented by the semi-transparent block.

We did not include an off-the-shelf connector on the board to connect to the control board since we wish to cut unnecessary weight and avoid imbalanced weight distribution. After assembly, the four signals of boosted voltage, the $2.5 \mathrm{~V}$, the "High Voltage Enable" of the booster, and the GND are presented at P1 on the front side. Four insulted copper wires from a transformer can be soldered between the power board and the control board to pass power and signal.

The completed power board is shown in Figure D-4. 

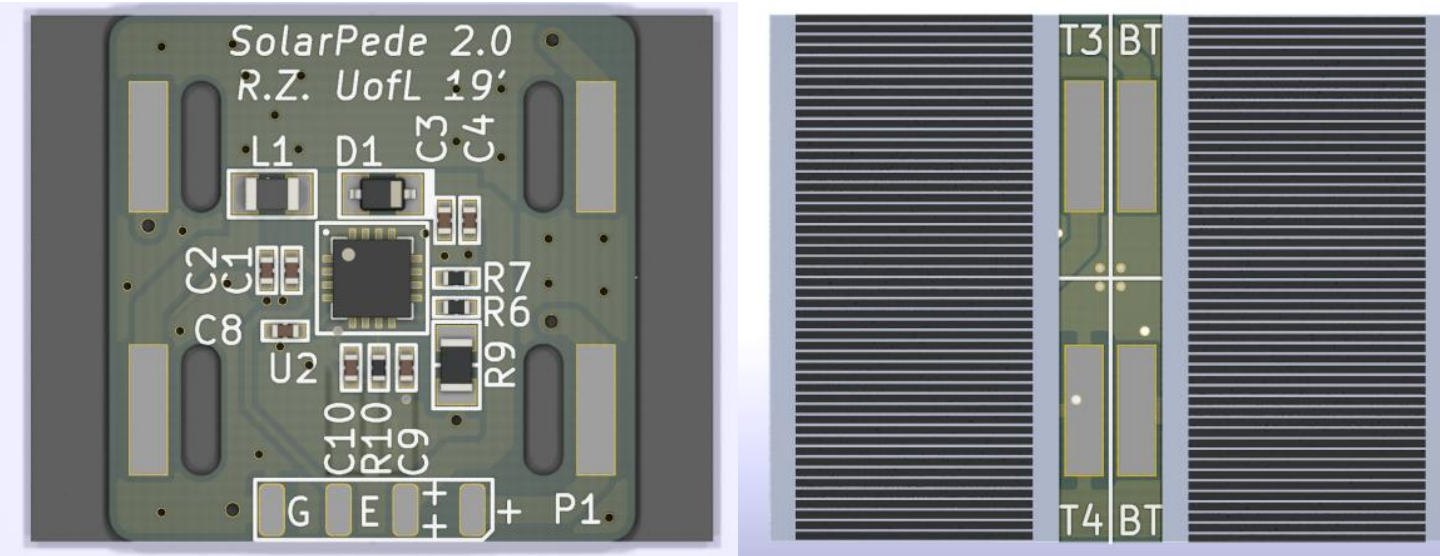

Figure D-4 Completed power board.

\section{D.2 Bluetooth MCU Selection and Configuration}

The circuity schematic of the control board is shown in Figure D-6, the PCB is a 2layers board.

A Bluetooth-enabled MCU module (BL652 from Laird®) was used as the on-board computer to generate gait sequence, monitoring solar cells voltage and receive user commands. We selected this Bluetooth module because it provides easy-to-use software and hardware packages so that we could quickly prototype the idea. The module is shown in Figure D-5.

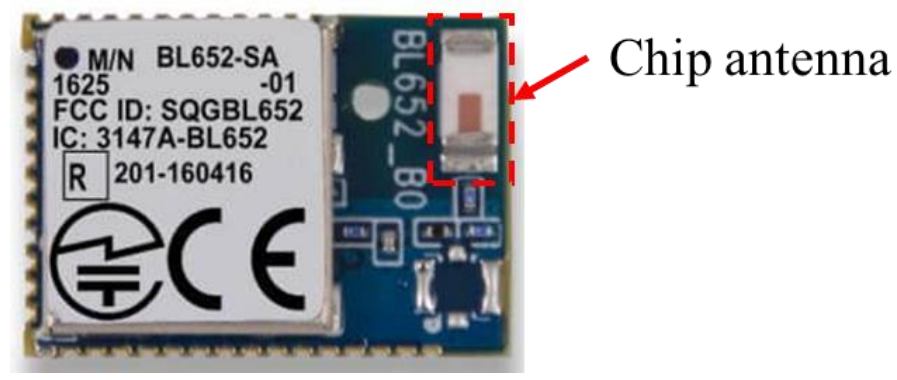

Figure D-5 BL652 Bluetooth MCU module with pre-assembled chip antenna (SA version).

Hardware-wise, the module includes all necessary electrical components, such as crystal oscillator, chip antenna and RF isolator all in an acceptably small footprint. These features contribute the research process by saving us time on minimum system and antenna 
debugging. Software-wise, an operating system (OS) implementing Bluetooth stack is already built into the firmware of the module with APIs provided. More importantly, the OS is packaged in the firmware, so the user only needs to develop the application code while without concerning the low-level task management. The drawback of the such arrangement is also obvious, we had to add one more PCB to the microrobot design, which gains extra weight to a weight-sensitive design. Second, the added weight may disturb weight balance and impede SolarPede's motion. Third, the software package cuts both ways; since there is no easy ways to access chip level configurations, the proposed PWM signaling method have to rely on delay function to achieve phase shifting, while the toggling speed is limited to $500 \mathrm{~Hz}$.

The microcontroller module was configured under development mode in the prototype, but it can be reconfigured into "Self-contained Run" mode by pull the "SIO_13/nAutoRun" pin to low. The module spends eight general purpose input/output pins (SIO pins) to toggle the MOSFET switches, they can be assigned as needed to accommodate PCB components placement. The MCU module monitors the high voltage supplied by the solar cells and the 2.5V , by analog input pins "SIO_04/AIN2" and "SIO_30/AN6". In case of an insufficient high voltage supply, the module can switch off the booster by toggle the pin "SIO_19". 


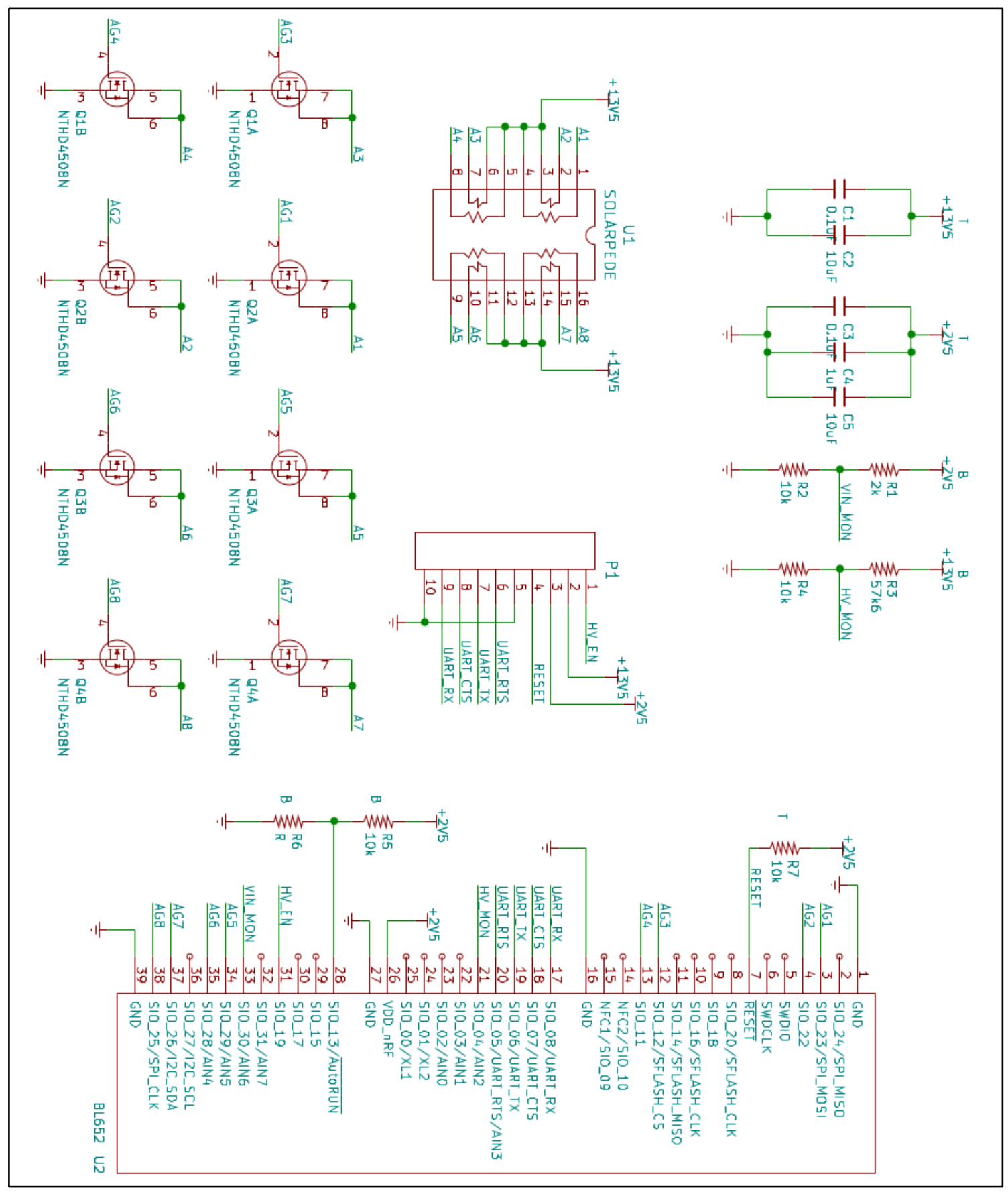

Figure D-6 Schematic of the control board. 


\section{D.3 Control Board Design and Construction}

The control board controls the gaiting sequence of the SolarPede. A 10-pin connector is placed on the edge of the board to receive supply voltage from the power board and provide access to the serial pins of the MCU to assist debugging the program before achieving full wireless operation. Indicated in Figure D-7 and Figure D-8 as P1. Notice the image in Figure D-8 is mirrored. Insolated transformer wires are directly soldered on it.

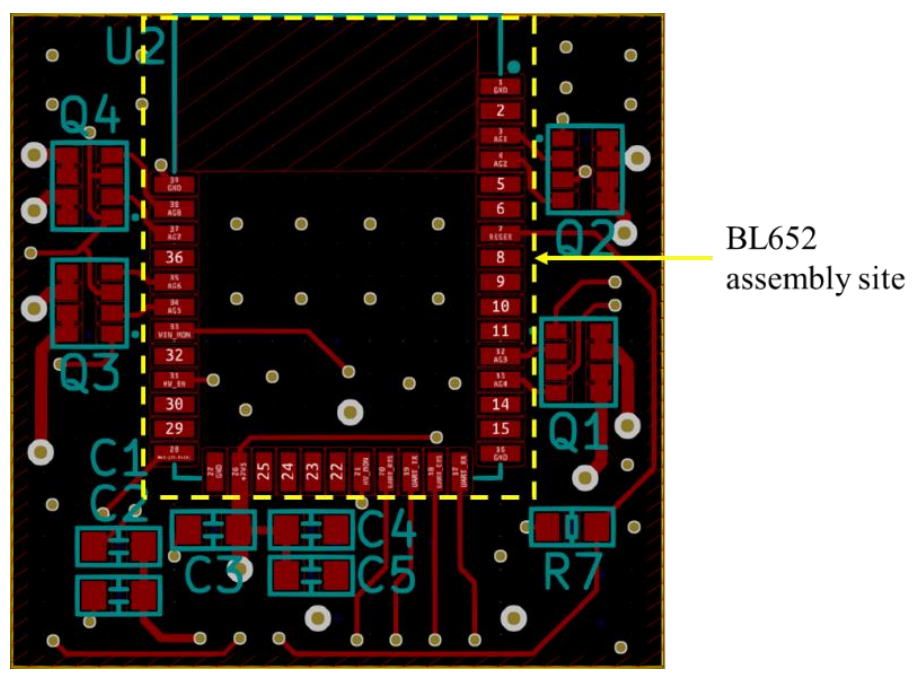

Figure D-7 Front layer of the control board.

All components on the front side of the control board including the MCU module, the MOSFETs, resistors, and capacitors are soldered first. The resistors on the bottom side are soldered sequentially. Since the wire-bonding process requires a flat and solid working surface, an aluminum fixture has a cavity with the exact same size and shape of the control board is used to support the assembled board for next wire-bonding process, as shown in Figure D-9. 


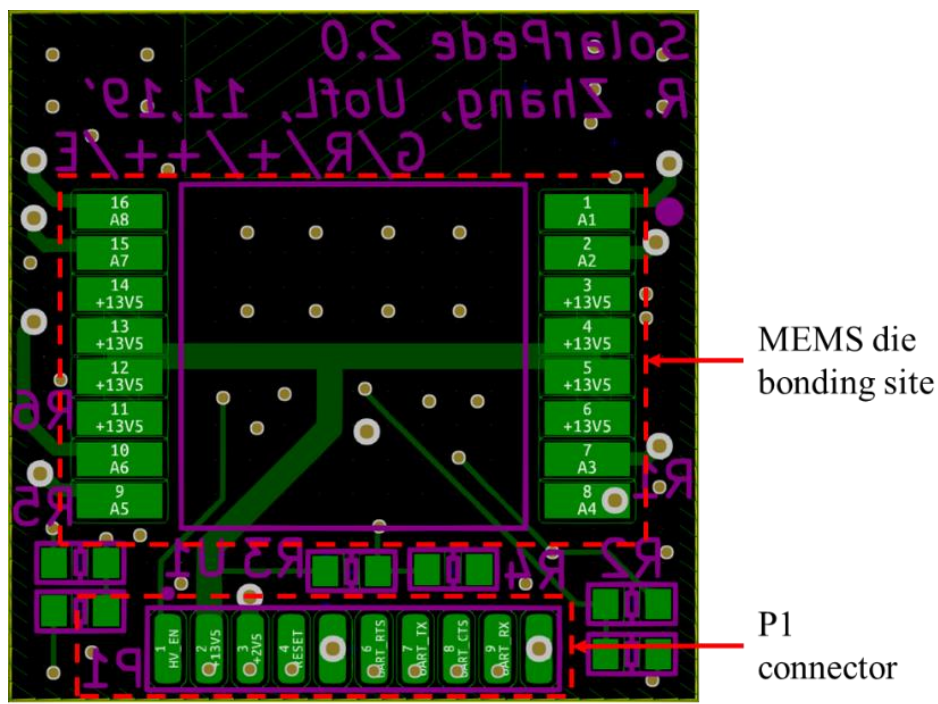

Figure D-8 Bottom side of the control board.

The MEMS die is firstly adhered to the bottom side of the PCB with super glue and loaded into the fixture, then wire bonded with corresponding bonding pads.

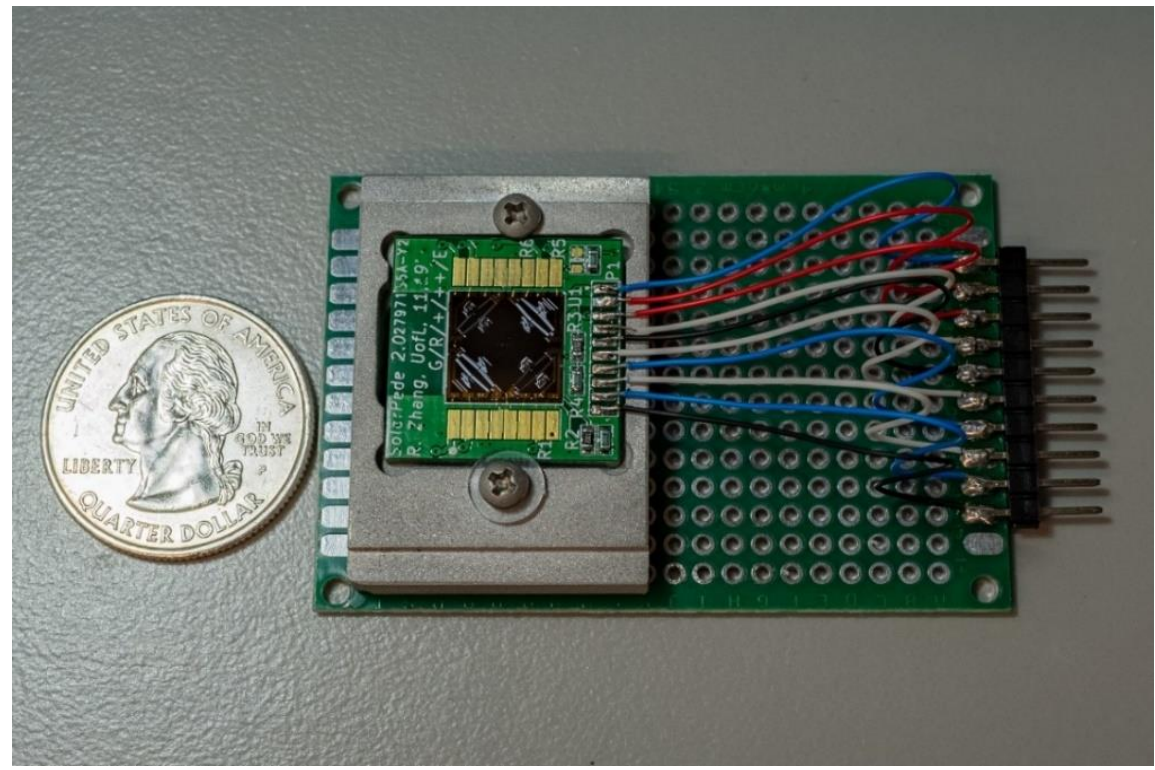

Figure D-9 Aluminum fixture of the control board to help wire-bonding. The same setup was also used in the prototype.

Figure D-10 shows the fully assembled control board. 

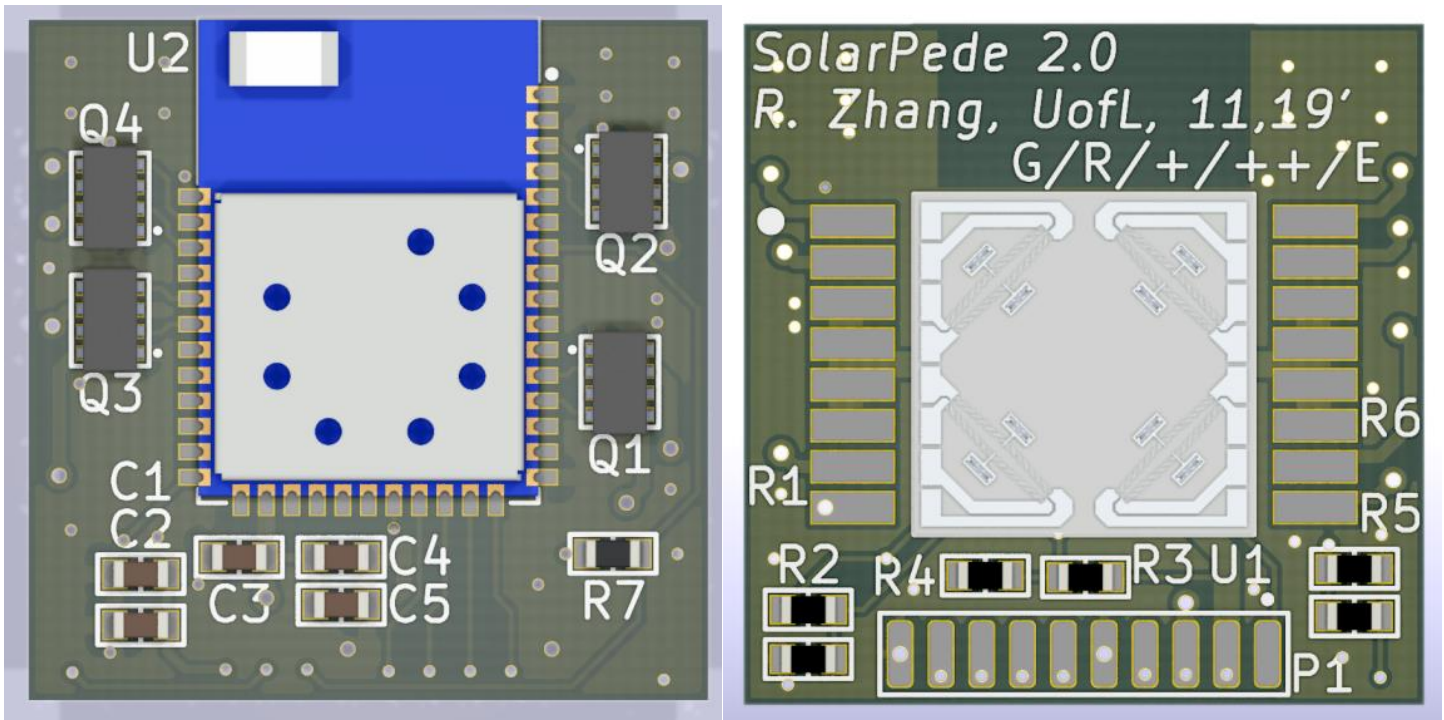

Figure D-10 Fully assembled control board, front and bottom side.

\section{D.5 SolarPede’s Gaiting Program}

The following code was used to generate gaiting sequence, the BL652 module was loaded with firmware version v28 960 r0. Laird provides UwTerminalX interface to load the script into the BL652 module, the software version is v1.10a.

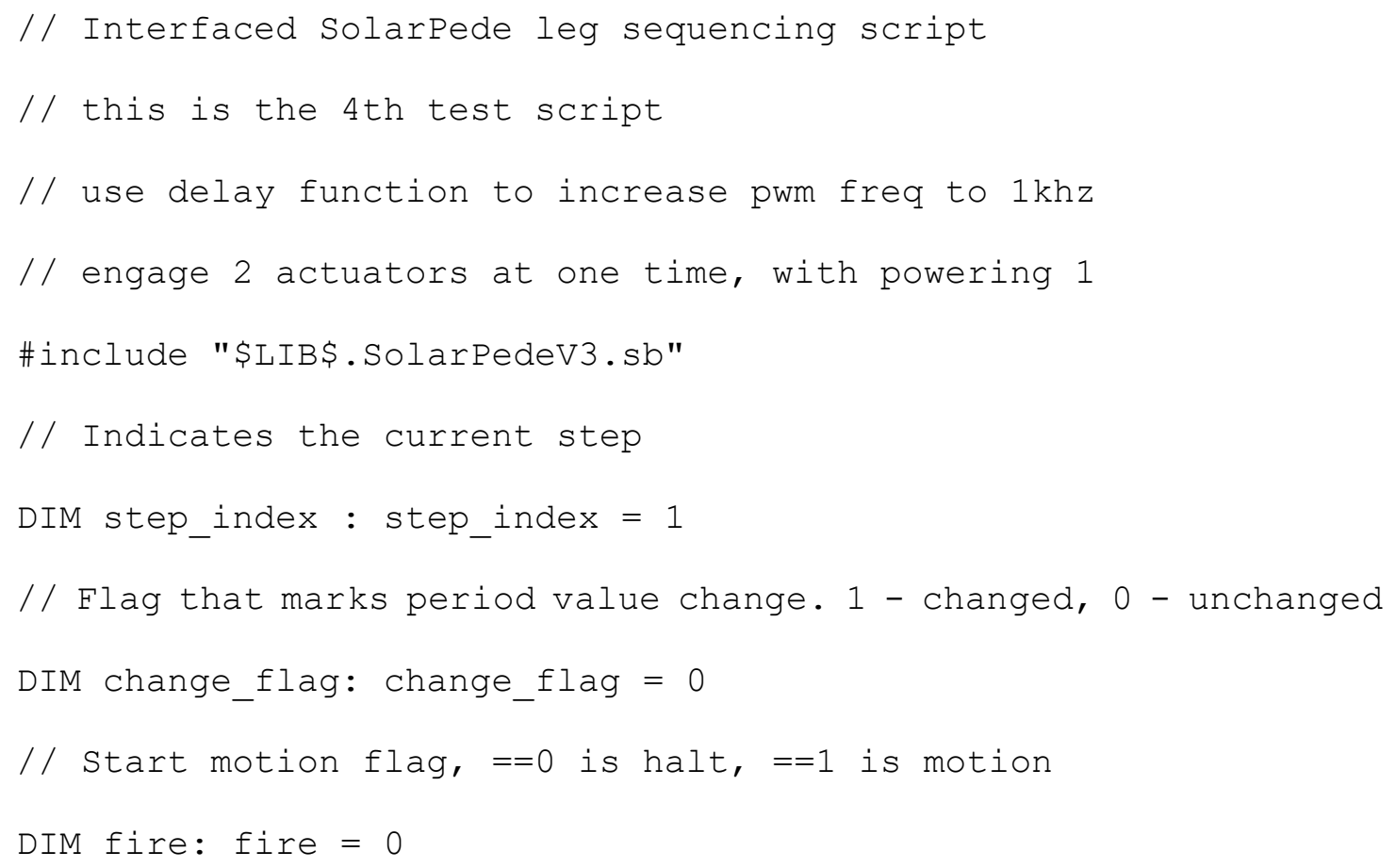


// New step size

DIM new_step

// If expecting more characters wait longer.

FUNCTION HndlrUartRx()

// Allow enough time for data to reach rx buffer Timerstart $(0,100,0)$

ENDFUNC 1

// User interface

FUNCTION HndlrTmro()

dim strLength, str\$, temp\$, temp

// Retrive the command

strLength=UartRead (str\$)

// Parse the user command

temp\$ = LEFT\$ $($ str\$, 1)

// Process "sxx" command - Period_step

if $\operatorname{strcmp}($ temp\$, $\operatorname{CMD} \$ 11])==0$ \&\& StrLen $(\operatorname{str} \$)>2$ then

StrShiftLeft (str\$, 1)

Period_step $=$ StrValDec (str\$)

print "\nNew Step Length set to: "; Period_step*2;"\n"

// change_flag $=1$

uartflush (01)

exitfunc 1

// Process command "1"

elseif strcmp (str\$, $\operatorname{CMD} \$ 1])==0$ then

print "Forward Motion Selected $\backslash n$ "

fire $=1$ 


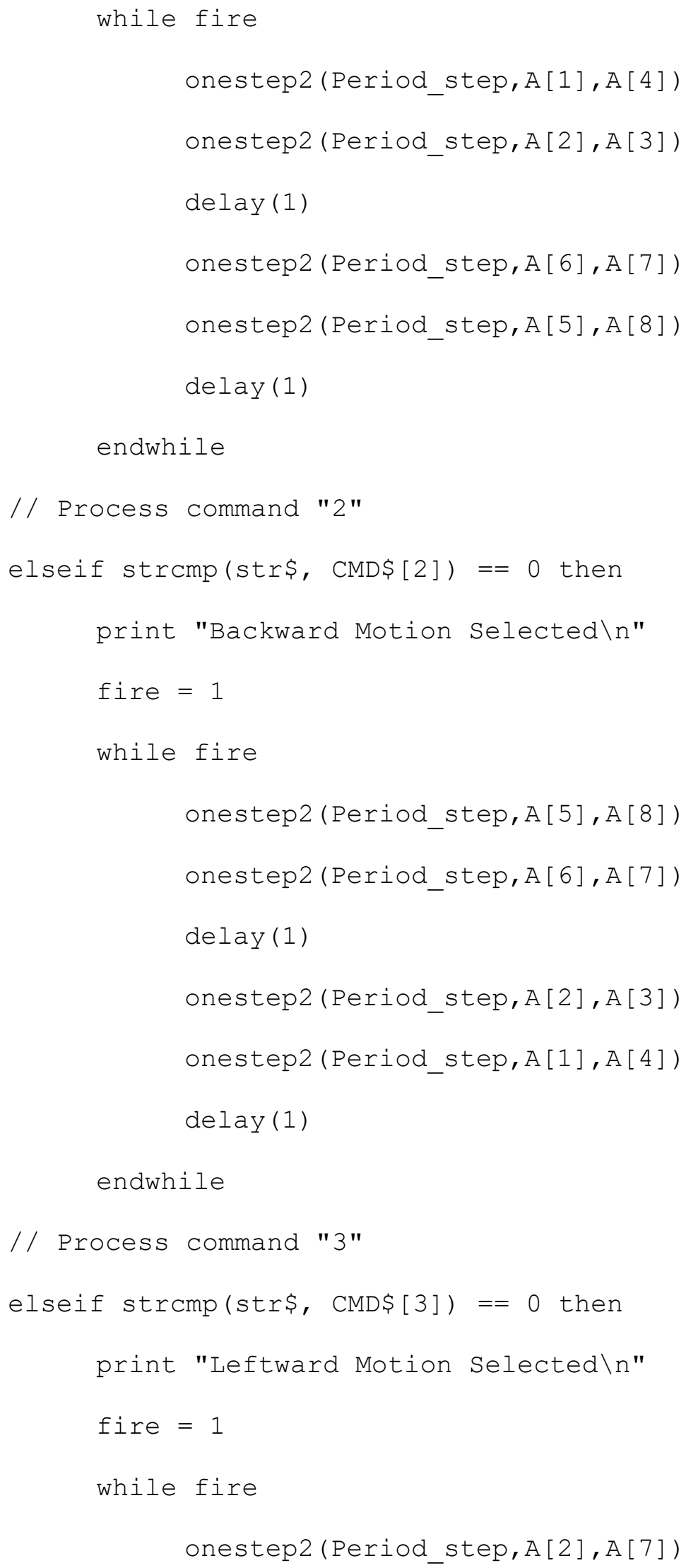




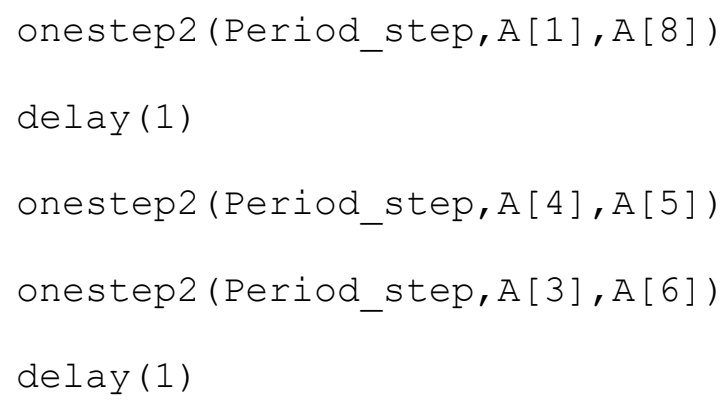




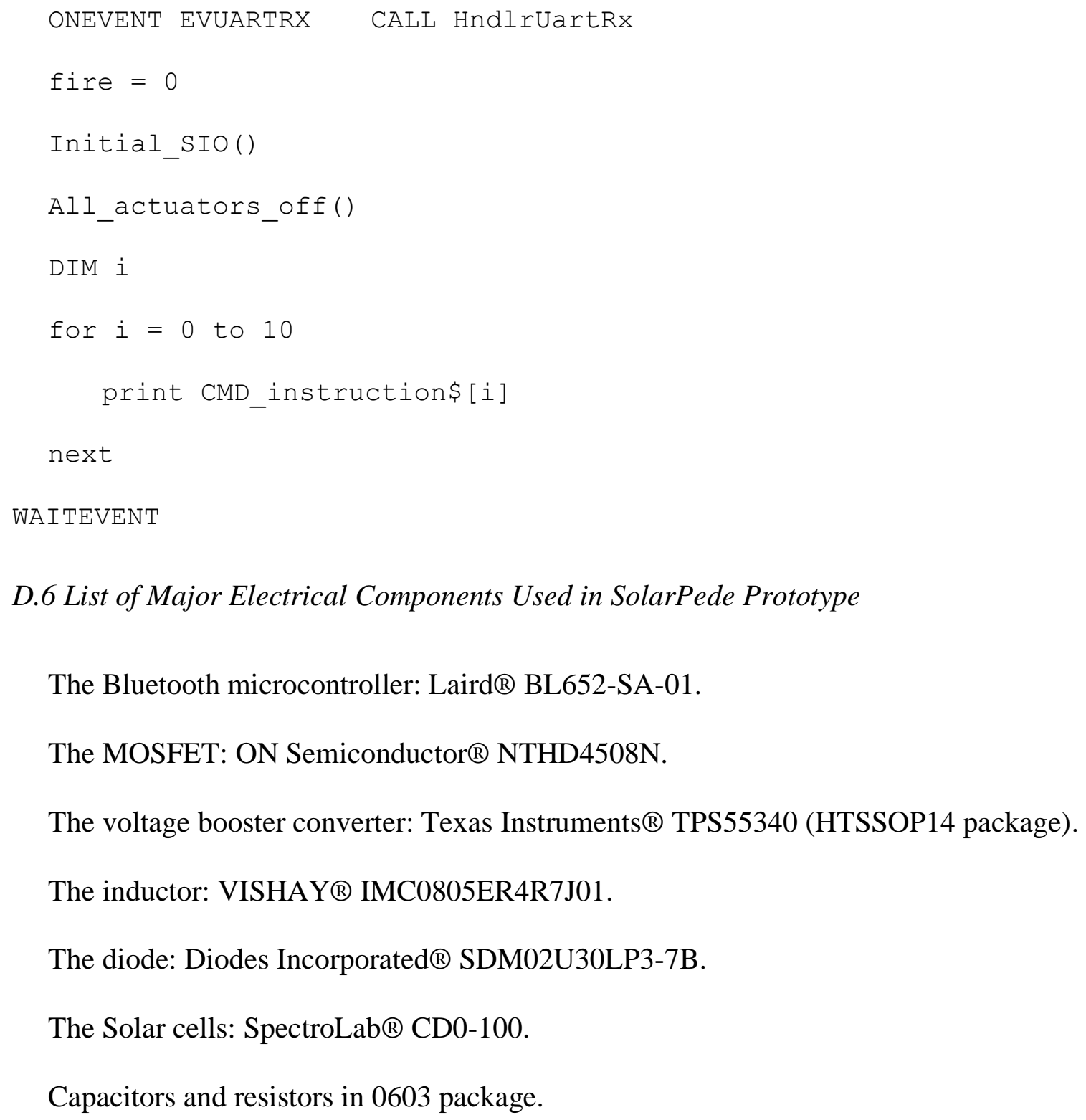




\section{APPENDIX E LIST OF PUBLICATIONS}

Journal Publications:

1) Design and Characterization of Solid Articulated Four Axes Microrobot for Microfactory Applications, Ruoshi Zhang, Andriy Sherehiy, Danming Wei and Dan Popa, Journal of Micro-Bio Robotics [70].

2) SolarPede: A Stick-and-Slip, Light-Powered, Mobile Micro-Crawler. Ruoshi Zhang, Jordan F. Klotz, Danming Wei, Zhong Yang, Andriy Sherehiy, Mohammad N. Saadatzi, Dan O. Popa, Journal of Micro-Bio Robotics.

Conference publications:

1) Design and Fabrication of an Articulated Four Axes Microrobot Arm. Ruoshi Zhang, Danming Wei, Zhong Yang, Dan Popa, SPIE 2017 [119].

2) Design, Analysis and Fabrication of sAFAM, a 4 DoF Assembled Microrobot, Ruoshi Zhang, Danming Wei, Dan Popa, MARSS 2018 [73], Won Best Student Paper Award.

3) ChevBot - An Untethered Microrobot Powered by Laser for Microfactory Applications, Ruoshi Zhang, Andriy Sherehiy, Zhong Yang, Danming Wei, Cindy K. Harnett, Dan Popa, ICRA 2019 [69].

4) Tracking Experiments with ChevBot: A Laser-Actuated Stick-Slip Microrobot, Ruoshi Zhang, Andriy Sherehiy, Danming Wei, Zhong Yang, M. Nasser Saadatzi, Dan O. Popa, MARSS 2019 [70], Won Best Student Paper Award 
5) Fabrication of Strain Gauge Based Sensors for Tactile Skins. Joshua Baptist, Ruoshi Zhang, Danming Wei, Dan Popa, SPIE 2017 [120].

6) Concept Validation for a Novel Stick-and-Slip, Light-Powered, Mobile MicroCrawler. J. F. Klotz, D. Wei, Z. Yang, R. Zhang, A. Sherehiy, M. N. Saadatzi and D. O. Popa, MARSS 2019 [113].

7) Multiphysics Model Validation Methodology for Laser Driven Microrobots. Z. Yang, M. N. Saadatzi, R. Zhang, A. Sherehiy, D. Wei, C. K. Harnett and D. O. Popa, CASE 2019 [114]. 
Zhang, Ruoshi, Ph.D.

Electrical and Computer Engineering Department

University of Louisville

Louisville, KY, 40292

Email: ruoshi.zhang@louisville.edu

Phone: (682) 582-0928

\section{Current Position}

Jan. 2016 - Present

EDUCATION

$2016-2020$

$2013-2015$

$2008-2012$

\section{Graduate Research Assistant}

Next Generation Systems Research Group

Department of Electrical and Computer Engineering

University of Louisville

Advisor: Dan O. Popa, Ph.D.

\section{Ph.D., Electrical Engineering}

University of Louisville, KY, USA

GPA: 3.747

Advisor: Dan O. Popa, Ph.D.

Thesis: Microrobots For Wafer Scale Microfactory:

Design, Fabrication, Integration and Control

\section{M.S., Electrical Engineering}

University of Texas at Arlington, TX, USA

GPA: 3.875

Advisor: Taylor Johnson, Ph.D.

Thesis: Model Based Design and Analysis of Automotive

Systems Using Time Triggered Controller Area Networks

\section{B.S., Electrical Engineering}

Tianjin University, Tianjin, China

GPA: 2.87

Senior Project: Audio Processing System Hardware Design Based on TMS320VC5416

\section{RESEARCH EMPLOYMENT}

Jan. 2016 - May 2020

Graduate Research Assistant, University of Louisville Advisor: Dan O. Popa, Ph.D. 
Project 1: Investigation of Ubiquitous Microrobots

Powered by Light

Funding Agency: National Science Foundation

Project 2: Multi-Modal Skin and Garments for Healthcare and Home Robots

Funding Agency: National Science Foundation

Sept. 2013 - May 2015 Graduate Research Assistant, University of Texas at Arlington

Advisor: Taylor Johnson, Ph.D.

Project 1: Reconfigurable Continuous-Culture Bioreactor.

Project 2: Model Based Design and Analysis of

Automotive Systems Using Time Triggered Controller

Area Networks.

TEACHING EXPERIENCE

Fall 2017

Graduate Teaching Assistant, University of Louisville

ECE 543/544 Fundamentals of Microfabrication and MEMS

Summer $2017 \quad$ Lecturer, University of Louisville

ECE 252, Introduction to Electrical Engineering

Spring 2017 Graduate Teaching Assistant, University of Louisville

ECE 333/334, Electronics I, Electronics I Lab

ECE 636, MEMS Design and Fabrication

Fall 2016

Graduate Teaching Assistant, University of Louisville ECE 543/544 Fundamentals of Microfabrication and MEMS

INDUSTRY EXPERIENCE

Mar. 2012 - Dec. 2012

Associate Hardware Engineer, Availink. Inc., Beijing, China

Printed circuit board design for digital and analog circuits.

Product developing and debugging.

\section{HONORS AND AWARDS}

Jul. 2019 Best Student Paper Award

Awarded for presentation of paper at IEEE Conference on Manipulation, Automation and Robotics at Small Scales (MARSS 2019) entitled: "Tracking Experiment with ChevBot: A Laser-Actuated Stick-Slip Microrobot".

Apr. 2019 Outstanding Graduate Student Award

This award is given to a student who has demonstrated outstanding research and scholarship in the Graduate Program in Electrical Engineering Department. 
Jul. 2018 Best Student Paper Award

Awarded for presentation of paper at IEEE Conference on Manipulation, Automation and Robotics at Small Scales (MARSS 2018) entitled: "Design, Analysis and Fabrication of sAFAM, a 4 DoF Assembled Microrobot".

Jun. 2017 Mobile Microrobotics Challenge (MMC) 2017

Finalist of IEEE Robotics and Automation Society, Mobile Microrobotics Challenge (MMC) 2017

Mar. 2014 Spirit of Innovation Competition

Awarded by US-India Chamber of Commerce ( $3^{\text {rd }}$ place) for ContinuousCulture Bioreactor Design.

\section{Professional Affiliations and Service}

Ad-hoc Reviewer

IEEE Conference on Manipulation, Automation and Robotics at Small Scales 2018

Professional Organization Member

Institute of Electrical and Electronics Engineers Student Member (IEEE)

International Society for Optics and Photonics (SPIE)

\section{WORKSHOPS AND POSTERS}

R. Zhang \& D. Popa, "Wafer-scale microfactories with assembled MEMS microrobots for nanotechnology applications," In Progress Toward Automated Micro-Bio-Nano Factories Through Robotic Manipulation Workshop, IEEE International Conference on Robotics and Automation (ICRA 2019), May 2019, Montreal, Canada.

R. Zhang, "Wafer-scale microfactories with assembled MEMS microrobots for nanotechnology applications," Poster at 2019 KY Nanotechnology and Additive Manufacturing Symposium, July 2019, Louisville, USA.

\section{STUDENTS MENTORED}

Summer 2019

Loic Vieille, UG student, École nationale supérieure de mécanique et des microtechniques

Summer 2018

John Ronkainen, UG student, University of Kentucky

Fall 2017 James Simmons, Bryan Knight, David Le, University of Louisville

Summer 2017 Daniel Goto, UG student, Washington State University

Summer 2016 Dustin Watts, UG student, Berea College

\section{Patent}

Baptist, J., Saadatzi, M. N., Yang, Z., Zhang, R., \& Popa, D. O. "Robot skin apparatus, method of fabricating a robot skin apparatus, and a system including a robot skin apparatus.” Patent No: PCT/US2018/026478. Publication Date: 11/10/2018 